Review

\title{
Chaetomugilins and Chaetoviridins-Promising Natural Metabolites: Structures, Separation, Characterization, Biosynthesis, Bioactivities, Molecular Docking, and Molecular Dynamics
}

\author{
Abdelsattar M. Omar 1,2,*(D), Gamal A. Mohamed ${ }^{3}(\mathbb{D})$ and Sabrin R. M. Ibrahim ${ }^{4,5, * \mathbb{D}}$ \\ 1 Department of Pharmaceutical Chemistry, Faculty of Pharmacy, King Abdulaziz University, \\ Jeddah 21589, Saudi Arabia \\ 2 Center for Artificial Intelligence in Precision Medicines, King Abdulaziz University, \\ Jeddah 21589, Saudi Arabia \\ 3 Department of Natural Products and Alternative Medicine, Faculty of Pharmacy, King Abdulaziz University, \\ Jeddah 21589, Saudi Arabia; gahussein@kau.edu.sa \\ 4 Department of Chemistry, Preparatory Year Program, Batterjee Medical College, Jeddah 21442, Saudi Arabia \\ 5 Department of Pharmacognosy, Faculty of Pharmacy, Assiut University, Assiut 71526, Egypt \\ * Correspondence: asmansour@kau.edu.sa (A.M.O.); sabrin.ibrahim@bmc.edu.sa or \\ sabreen.ibrahim@pharm.aun.edu.eg (S.R.M.I.); Tel.: +966-56-768-1466 (A.M.O.); +966-58-118-3034 (S.R.M.I.)
}

check for

updates

Citation: Omar, A.M.; Mohamed,

G.A.; Ibrahim, S.R.M

Chaetomugilins and

Chaetoviridins-Promising Natural Metabolites: Structures, Separation, Characterization, Biosynthesis,

Bioactivities, Molecular Docking, and Molecular Dynamics. J. Fungi 2022, 8 , 127. https://doi.org/10.3390/ jof8020127

Academic Editor: Laurent Dufossé

Received: 21 December 2021

Accepted: 24 January 2022

Published: 27 January 2022

Publisher's Note: MDPI stays neutral with regard to jurisdictional claims in published maps and institutional affiliations.

Copyright: (C) 2022 by the authors. Licensee MDPI, Basel, Switzerland. This article is an open access article distributed under the terms and conditions of the Creative Commons Attribution (CC BY) license (https:// creativecommons.org/licenses/by/ $4.0 /)$.

\begin{abstract}
Fungi are recognized as luxuriant metabolic artists that generate propitious biometabolites Historically, fungal metabolites have largely been investigated as leads for various therapeutic agents. Chaetomugilins and the closely related chaetoviridins are fungal metabolites, and each has an oxygenated bicyclic pyranoquinone core. They are mainly produced by various Chaetomaceae species. These metabolites display unique chemical features and diversified bioactivities. The current review gives an overview of research about fungal chaetomugilins and chaetoviridins regarding their structures, separation, characterization, biosynthesis, and bioactivities. Additionally, their antiviral potential towards the SARS-CoV-2 protease was evaluated using docking studies and molecular dynamics (MD) simulations. We report on the docking and predictive binding energy estimations using reported crystal structures of the main protease (PDB ID: 6M2N, 6W81, and 7K0f) at variable resolutions-i.e., 2.20, 1.55, and $1.65 \AA$, respectively. Chaetovirdin D (43) exhibited highly negative docking scores of $-7.944,-8.141$, and $-6.615 \mathrm{kcal} / \mathrm{mol}$, when complexed with $6 \mathrm{M} 2 \mathrm{~N}, 6 \mathrm{~W} 81$, and 7K0f, respectively. The reference inhibitors exhibited the following scores: $-5.377,-6.995$, and $-8.159 \mathrm{kcal} / \mathrm{mol}$, when complexed with $6 \mathrm{M} 2 \mathrm{~N}, 6 \mathrm{~W} 81$, and 7K0f, respectively. By using molecular dynamics simulations, chaetovirdin D's stability in complexes with the viral protease was analyzed, and it was found to be stable over the course of $100 \mathrm{~ns}$.
\end{abstract}

Keywords: chaetomugilins; chaetoviridins; fungi; Chaetomaceae; characterization; bioactivities; molecular docking; COVID-19; protease; biosynthesis

\section{Introduction}

Fungi are a wealthy and substantial pool of many secondary metabolites with many different structures and diversified bioactivities [1-11]. These metabolites attract much attention as lead metabolites for pharmaceutical agents, and for plant protection [1,8-16]. Fungal polyketides (FPKs) represent one of the largest and most structurally diverse groups of fungal metabolites. They range from simple and aromatic to highly macrocyclic and complex $[10,13,17]$. Their backbone is biosynthesized by the condensation of acyl-CoA thioesters [18]. Their structural variations originate from differences in the starting and extending units, methylation pattern, chain length, degree of reduction, and modifications by tailoring enzymes [19]. Mycotoxins and pigments are among the FPKs that have had 
remarkable contributions in the field of drug discovery [20]. Azaphilones (azaphilonoids or isochromenes) are fungal pigments belonging to FPKs. Structurally, they have an isochromene skeleton that contains an oxygenated bicyclic pyrano-quinone core and a quaternary carbon center [21]. Biosynthetically, the $\mathrm{O}$ atom in the pyran chromophore could be exchanged by an $\mathrm{N}$ atom in the existence of primary amines, and accordingly, the pigment color will shift to red [22]. They are produced by various basidiomycetous and ascomycetous fungi, including Chaetomium, Penicillium, Aspergillus, Talaromyces, Phomopsis, Monascus, Emericella, Epicoccum, Hypoxylon, and Pestalotiopsis, where they are accountable for the green, red, or yellow color of mycelia and/or fruiting bodies [23]. They possess myriad bioactivities: antitumor, cytotoxic, antimicrobial, anti-inflammatory, antioxidant, enzyme inhibitory, antiviral, insecticidal, and antileishmanial [24]. Chaetomugilins and closely related chaetoviridins are azaphilones featuring a C-7-methyl group and C-5 chlorineexcept for chaetomugilins T (29) and U (30) - and a C-3-branched pentenyl chain (Figure 1). However, chaetomugiline P (24) differs from the others in that it has no substituent at C-7 and a methyl group at C-5. 3-Methyl-4-hydroxy-1-pentyl chains at C-3 are found in some chaetomugilins and chaetoviridins. Sometimes, they bear a five-membered lactone and/or a fused tetrahydrofuran / $\delta$-lactone [25]. The 7-OH group can have $(\mathrm{S})$ or $(\mathrm{R})$ configuration, though (7S) isomers are the most common among these metabolites. On the other hand, the 7-hydroxyl can be part of a furanone ring [26]. These fungal metabolites are produced by various Chaetomium species. Chaetoviridins were firstly reported by Takahashi et al. from Chaetomium globosum var. flavoviride [27]. Chaetomugilins are known as cytotoxic metabolites, whereas chaetoviridins have antifungal and antibiotic activities [28]. Recently, these metabolites have been recognized as a unique family of fungal metabolites in view of their interesting structural features and prominent bioactivities, which could provoke enormous attention from natural products chemists and pharmacologists. The current review focuses on chaetomugilins and chaetoviridins from fungal sources, including isolation, structural characterization, biosynthesis, and bioactivities (Tables 1 and 2). Some of the metabolites have been reported with the same names, despite having different structures and molecular formulae-e.g., chaetomugilin S, chaetoviridin B, and chaetoviridin G. Moreover, the structures of some compounds have been revised and renamed: in such cases, both structures have been drawn in the figures, highlighting the new names and corresponding references. Additionally, the emergence of the COVID-19 pandemic motivated us to investigate the potential of these metabolites as antiviral agents towards SARS-CoV-2 using docking studies and molecular dynamics (MD) simulations. Literature searching was carried out using diverse databases-Web of Science, PubMed (MedLine), GoogleScholar, Scopus, and SciFinder-and different publishers- Springer-Link (Cham, Switzerland), Wiley (New York, NY, United States), Taylor \& Francis (London, England), Bentham (Sharjah, United Arab Emirates), and ACS (Washington, DC, USA) Publications. 


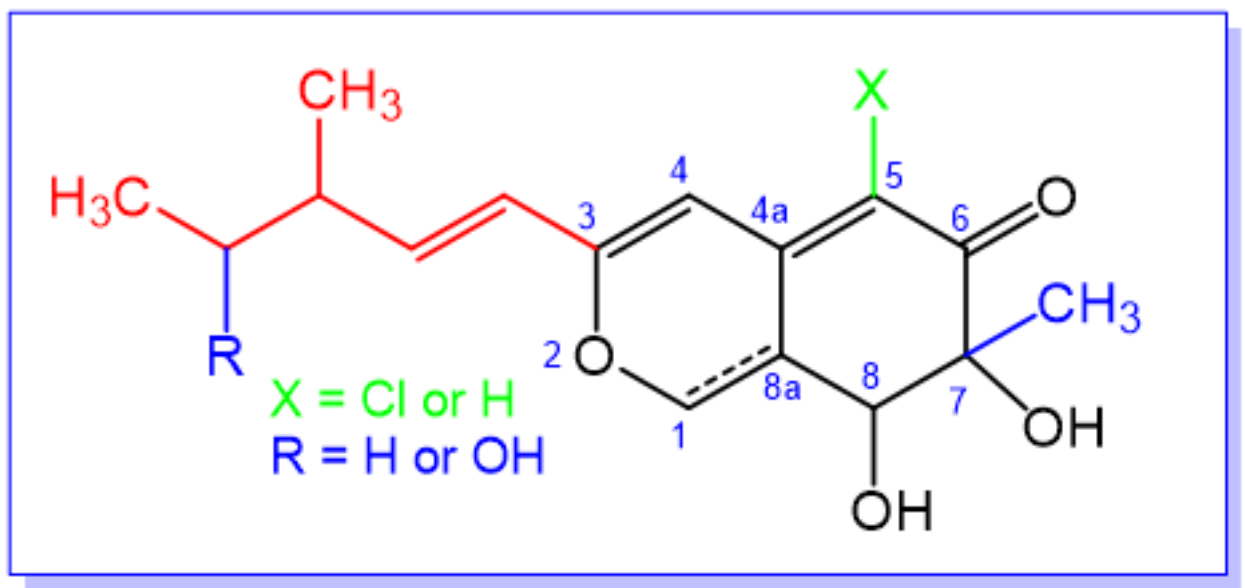

Figure 1. The basic skeleton of chaetomugilins and chaetoviridins.

\section{Extraction, Isolation, and Structural Characterization}

For isolation of these metabolites, the fungal materials were extracted with EtOAc or $\mathrm{MeOH}$. Their presence in the extract can be detected by TLC using the solvent systems: EtOAc:toluene:AcOH:formic acid 2.5:7.5:1:1, $\mathrm{CHCl}_{3}: \mathrm{MeOH}: \mathrm{AcOH}$ 95:5:0.1, or petroleum spirit/EtOAc 40:60. Upon development, the spots on the $\mathrm{SiO}_{2}$ TLC plates can be visualized as dark spots under ultraviolet light $(365 \mathrm{~nm}$ and $254 \mathrm{~nm}$ ), or by using ammonia (red color) or ceric ammonium molybdate stain (dark spots; $\mathrm{H}_{2} \mathrm{O}(90 \mathrm{~mL}), \mathrm{H}_{2} \mathrm{SO}_{4}(10 \mathrm{~mL})$, ammonium molybdate (2.5 g), cerium (IV) sulfate (1.0 g)) [27,29-31]. Moreover, they gave positive Beilstein reactions [27]. The EtOAc or MeOH extract can be separated by column chromatography using $\mathrm{SiO}_{2} \mathrm{CC}$ (PE:acetone 9:1-6.5:3.5 or 40:1-2:1; $\mathrm{CH}_{2} \mathrm{Cl}_{2}$ : $\mathrm{MeOH}$; cyclohexane:MeOH; EtOAc: $\mathrm{CH}_{2} \mathrm{Cl}_{2}$; cyclohexane:EtOAc; $\mathrm{CHCl}_{3}: \mathrm{MeOH}$ gradient), Sephadex LH-20 (MeOH; $\mathrm{CH}_{2} \mathrm{Cl}_{2}: \mathrm{MeOH}$ 6:4; $\mathrm{CH}_{2} \mathrm{Cl}_{2}: \mathrm{MeOH}$ 1:1; $\mathrm{CHCl}_{3}: \mathrm{MeOH} 1: 1$ ), or MPLC (C-18 ODS) (MeOH: $\mathrm{H}_{2} \mathrm{O}$ gradient) [25,31-33]. Finally, the fractions or isolated compounds can be further purified by preparative HPLC (MeOH: $\mathrm{H}_{2} \mathrm{O} 90 \%, 85 \%, 75 \%, 65 \%$, or 50\% v/v), $\mathrm{CH}_{3} \mathrm{CN} / \mathrm{H}_{2} \mathrm{O}(60: 40,65: 35$, or 75:25, v/v) [31-35], or normal phase HPLC (n-hexane:EtOAc 7:3, 4:1, 1:4, 2:1, 1: 3, or 3:2) [36]. Their isolation can also be achieved by loading the sample onto $\mathrm{TLC} \mathrm{SiO}_{2}$ plates in the form of uniform bands. 
Table 1. List of chaetomugilins and chaetoviridins. (Molecular weight and formula, fungal source, host, and place of discovery).

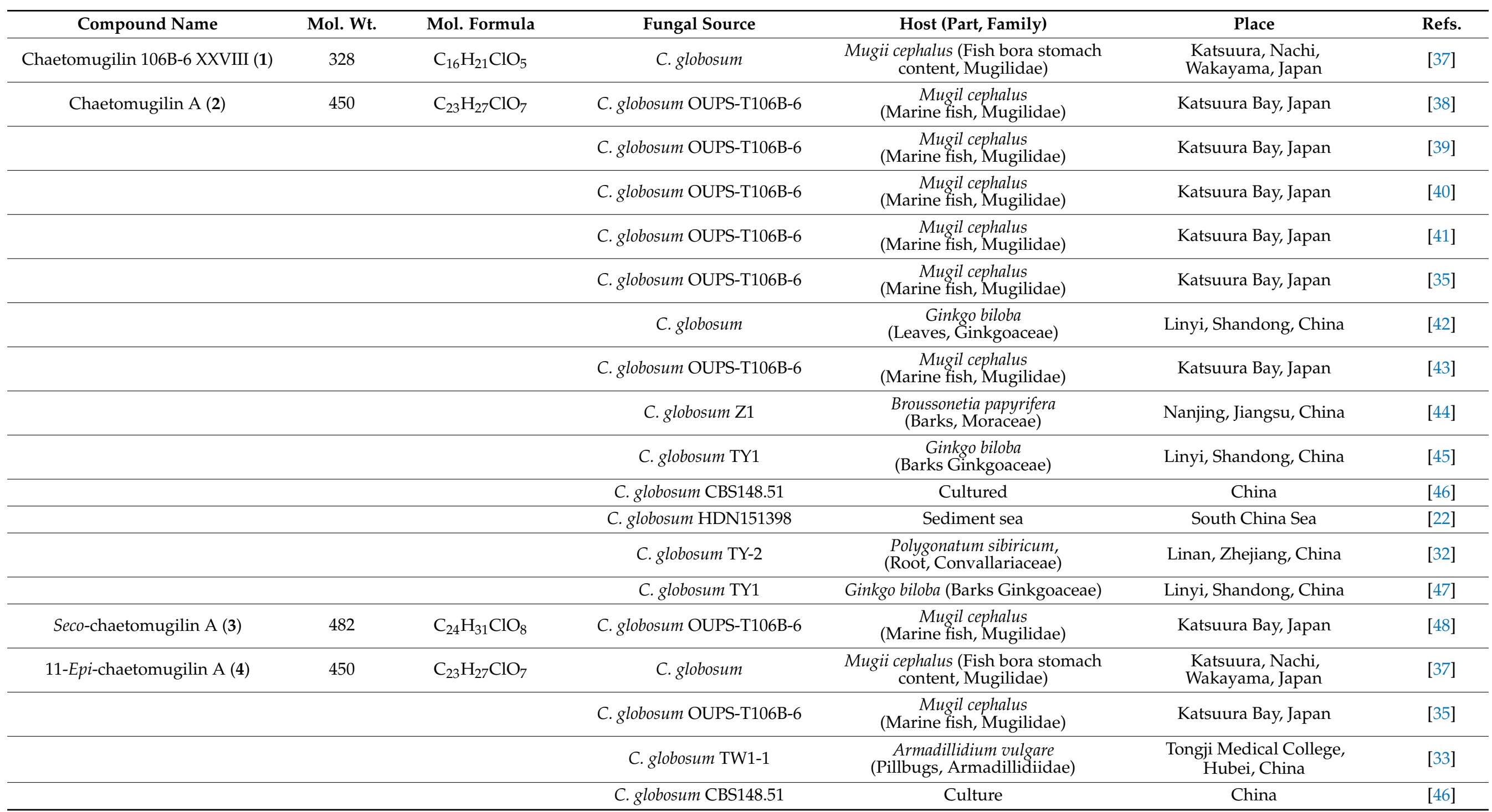


Table 1. Cont.

\begin{tabular}{|c|c|c|c|c|c|c|}
\hline Compound Name & Mol. Wt. & Mol. Formula & Fungal Source & Host (Part, Family) & Place & Refs. \\
\hline \multirow[t]{2}{*}{$4^{\prime}$-Epi-chaetomugilin A (5) } & 450 & $\mathrm{C}_{23} \mathrm{H}_{27} \mathrm{ClO}_{7}$ & C. globosum & $\begin{array}{l}\text { Mugii cephalus (Fish bora stomach } \\
\text { content, Mugilidae) }\end{array}$ & $\begin{array}{l}\text { Katsuura, Nachi, } \\
\text { Wakayama, Japan }\end{array}$ & [37] \\
\hline & & & C. globosum OUPS-T106B-6 & $\begin{array}{c}\text { Mugil cephalus } \\
\text { (Marine fish, Mugilidae) }\end{array}$ & Katsuura Bay, Japan & {$[35]$} \\
\hline \multirow[t]{3}{*}{ Chaetomugilin B (6) } & 464 & $\mathrm{C}_{24} \mathrm{H}_{29} \mathrm{ClO}_{7}$ & C. globosum OUPS-T106B-6 & $\begin{array}{c}\text { Mugil cephalus } \\
\text { (Marine fish, Mugilidae) }\end{array}$ & Katsuura Bay, Japan & {$[38]$} \\
\hline & & & C. globosum OUPS-T106B-6 & $\begin{array}{c}\text { Mugil cephalus } \\
\text { (Marine fish, Mugilidae) }\end{array}$ & Katsuura Bay, Japan & [39] \\
\hline & & & C. globosum Z1 & $\begin{array}{l}\text { Broussonetia papyrifera } \\
\text { (Barks, Moraceae) }\end{array}$ & Nanjing, Jiangsu, China & {$[44]$} \\
\hline \multirow[t]{3}{*}{ Chaetomugilin C (7) } & 432 & $\mathrm{C}_{23} \mathrm{H}_{25} \mathrm{ClO}_{6}$ & C. globosum OUPS-T106B-6 & $\begin{array}{c}\text { Mugil cephalus } \\
\text { (Marine fish, Mugilidae) }\end{array}$ & Katsuura Bay, Japan & {$[38]$} \\
\hline & & & C. globosum OUPS-T106B-6 & $\begin{array}{c}\text { Mugil cephalus } \\
\text { (Marine fish, Mugilidae) }\end{array}$ & Katsuura Bay, Japan & [39] \\
\hline & & & C. globosum OUPS-T106B-6 & $\begin{array}{c}\text { Mugil cephalus } \\
\text { (Marine fish, Mugilidae) }\end{array}$ & Katsuura Bay, Japan & [40] \\
\hline \multirow[t]{8}{*}{ Chaetomugilin D (8) } & 434 & $\mathrm{C}_{23} \mathrm{H}_{27} \mathrm{ClO}_{6}$ & C. globosum & $\begin{array}{l}\text { Adiantum capillus-veneris } \\
\text { (Plant, Pteridaceae) }\end{array}$ & $\begin{array}{l}\text { Saint Katherine Protectorate, } \\
\text { Sinai, Egypt }\end{array}$ & [49] \\
\hline & & & C. globosum OUPS-T106B-6 & $\begin{array}{c}\text { Mugil cephalus } \\
\text { (Marine fish, Mugilidae) }\end{array}$ & Katsuura Bay, Japan & [39] \\
\hline & & & C. globosum OUPS-T106B-6 & $\begin{array}{c}\text { Mugil cephalus } \\
\text { (Marine fish, Mugilidae) }\end{array}$ & Katsuura Bay, Japan & [40] \\
\hline & & & C. globosum & Ginkgo bilob (Leaves, Ginkgoaceae) & Linyi, Shandong province, China & [42] \\
\hline & & & C. globosum OUPS-T106B-6 & $\begin{array}{c}\text { Mugil cephalus } \\
\text { (Marine fish, Mugilidae) }\end{array}$ & Katsuura Bay, Japan & [41] \\
\hline & & & C. globosum OUPS-T106B-6 & $\begin{array}{c}\text { Mugil cephalus } \\
\text { (Marine fish, Mugilidae) }\end{array}$ & Katsuura Bay, Japan & [43] \\
\hline & & & C. globosum DAOM 240359 & $\begin{array}{l}\text { Indoor air samples } \\
\text { or building materials }\end{array}$ & $\begin{array}{l}\text { Ontario, Alberta, Saskatchewan, } \\
\text { Nova Scotia, Canada }\end{array}$ & {$[30]$} \\
\hline & & & C. globosum DAOM 240359 & $\begin{array}{l}\text { Indoor air samples } \\
\text { or building materials }\end{array}$ & $\begin{array}{l}\text { Ontario, Alberta, Saskatchewan, } \\
\text { Nova Scotia, Canada }\end{array}$ & [50] \\
\hline
\end{tabular}


Table 1. Cont.

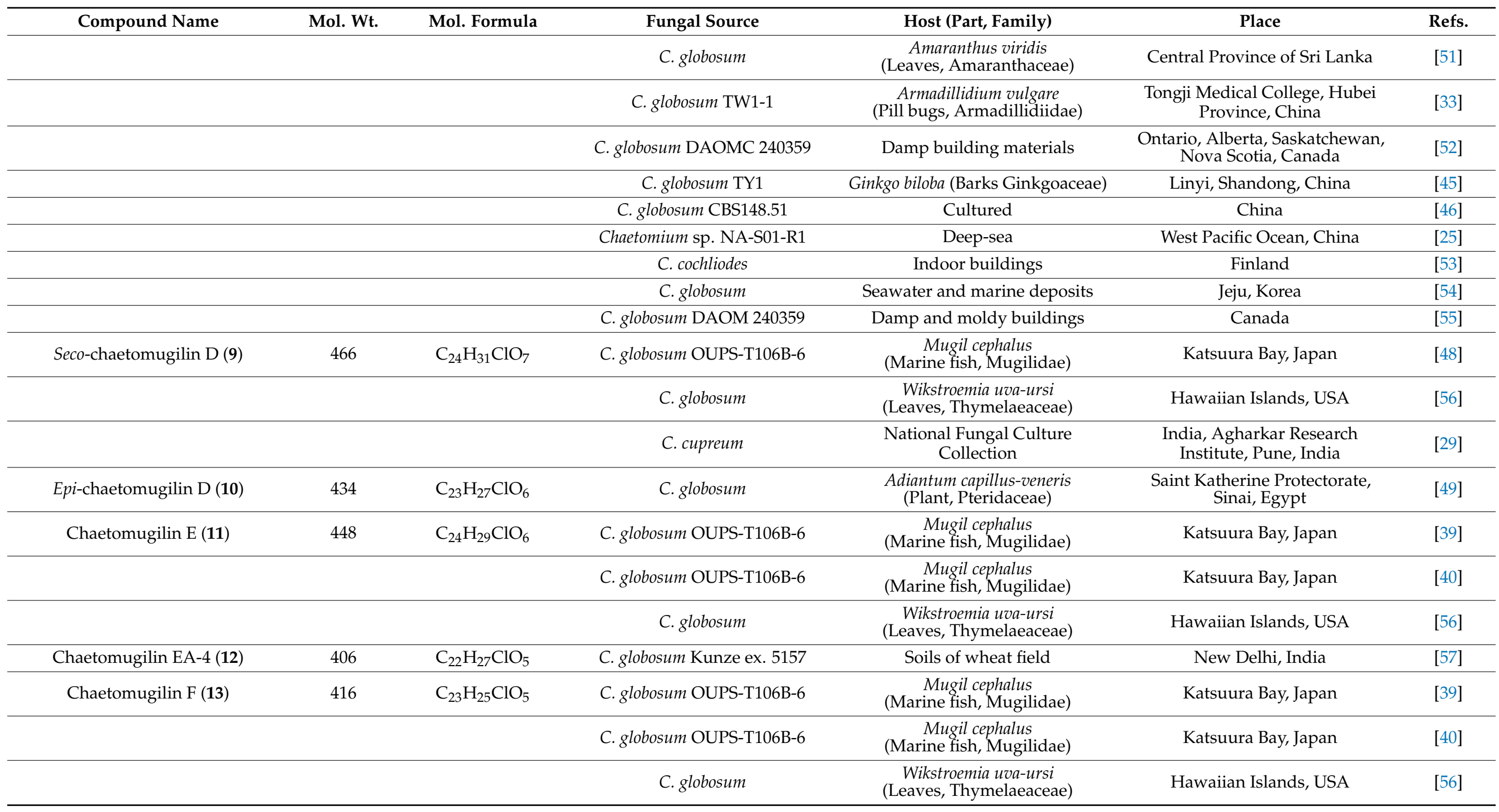


Table 1. Cont.

\begin{tabular}{|c|c|c|c|c|c|c|}
\hline Compound Name & Mol. Wt. & Mol. Formula & Fungal Source & Host (Part, Family) & Place & Refs. \\
\hline \multirow[t]{2}{*}{ Chaetomugilin G (14) } & 464 & $\mathrm{C}_{24} \mathrm{H}_{29} \mathrm{ClO}_{7}$ & C. globosum OUPS-T106B-6 & $\begin{array}{c}\text { Mugil cephalus } \\
\text { (Marine fish, Mugilidae) }\end{array}$ & Katsuura Bay, Japan & [39] \\
\hline & & & C. globosum OUPS-T106B-6 & $\begin{array}{c}\text { Mugil cephalus } \\
\text { (Marine fish, Mugilidae) }\end{array}$ & Katsuura Bay, Japan & [41] \\
\hline \multirow[t]{2}{*}{ Chaetomugilin H (15) } & 448 & $\mathrm{C}_{24} \mathrm{H}_{29} \mathrm{ClO}_{6}$ & C. globosum OUPS-T106B-6 & $\begin{array}{c}\text { Mugil cephalus } \\
\text { (Marine fish, Mugilidae) }\end{array}$ & Katsuura Bay, Japan & [39] \\
\hline & & & C. globosum OUPS-T106B-6 & $\begin{array}{c}\text { Mugil cephalus } \\
\text { (Marine fish, Mugilidae) }\end{array}$ & Katsuura Bay, Japan & [41] \\
\hline \multirow{6}{*}{ Chaetomugilin I (16) } & & & C. globosum & $\begin{array}{c}\text { Mugil cephalus } \\
\text { (Marine fish, Mugilidae) }\end{array}$ & Katsuura Bay, Japan & [58] \\
\hline & & & C. globosum & $\begin{array}{c}\text { Mugil cephalus } \\
\text { (Marine fish, Mugilidae) }\end{array}$ & Katsuura Bay, Japan & [58] \\
\hline & & & C. globosum & $\begin{array}{c}\text { Wikstroemia uva-ursi } \\
\text { (Leaves, Thymelaeaceae) }\end{array}$ & Hawaiian Islands, USA & [56] \\
\hline & & & C. globosum TY1 & Ginkgo biloba (Barks Ginkgoaceae) & Linyi, Shandong, China & [45] \\
\hline & & & C. globosum TY1 & Ginkgo biloba (Barks, Ginkgoaceae) & Linyi, Shandong, China & [47] \\
\hline & & & C. globosum Kunze ex. 5157 & Soils of wheat field & New Delhi, India & [57] \\
\hline \multirow[t]{2}{*}{ 11-Epi-chaetomugilin I (17) } & 406 & $\mathrm{C}_{22} \mathrm{H}_{27} \mathrm{ClO}_{5}$ & C. globosum OUPS-T106B-6 & $\begin{array}{l}\text { Mugil cephalus (Marine fish, } \\
\text { Mugilidae) }\end{array}$ & Katsuura Bay, Japan & [59] \\
\hline & & & C. globosum & $\begin{array}{l}\text { Wikstroemia uva-ursi (Leaves, } \\
\text { Thymelaeaceae) }\end{array}$ & Hawaiian Islands, USA & [56] \\
\hline \multirow[t]{5}{*}{ Chaetomugilin J (18) } & 390 & $\mathrm{C}_{22} \mathrm{H}_{27} \mathrm{ClO}_{4}$ & C. globosum & $\begin{array}{c}\text { Mugil cephalus } \\
\text { (Marine fish, Mugilidae) }\end{array}$ & Katsuura Bay, Japan & [58] \\
\hline & & & C. globosum & $\begin{array}{l}\text { Adiantum capillus-veneris } \\
\text { (Plant, Pteridaceae) }\end{array}$ & $\begin{array}{l}\text { Saint Katherine Protectorate, } \\
\text { Sinai, Egypt }\end{array}$ & [49] \\
\hline & & & C. globosum & $\begin{array}{l}\text { Mugii cephalus (Fish bora stomach } \\
\text { content, Mugilidae) }\end{array}$ & $\begin{array}{c}\text { Katsuura, Nachi, } \\
\text { Wakayama, Japan }\end{array}$ & [37] \\
\hline & & & C. globosum & $\begin{array}{c}\text { Amaranthus viridis } \\
\text { (Leaves, Amaranthaceae) }\end{array}$ & Central Province of Sri Lanka & {$[51]$} \\
\hline & & & C. globosum & $\begin{array}{c}\text { Wikstroemia uva-ursi } \\
\text { (Leaves, Thymelaeaceae) }\end{array}$ & Hawaiian Islands, USA & [56] \\
\hline
\end{tabular}


Table 1. Cont.

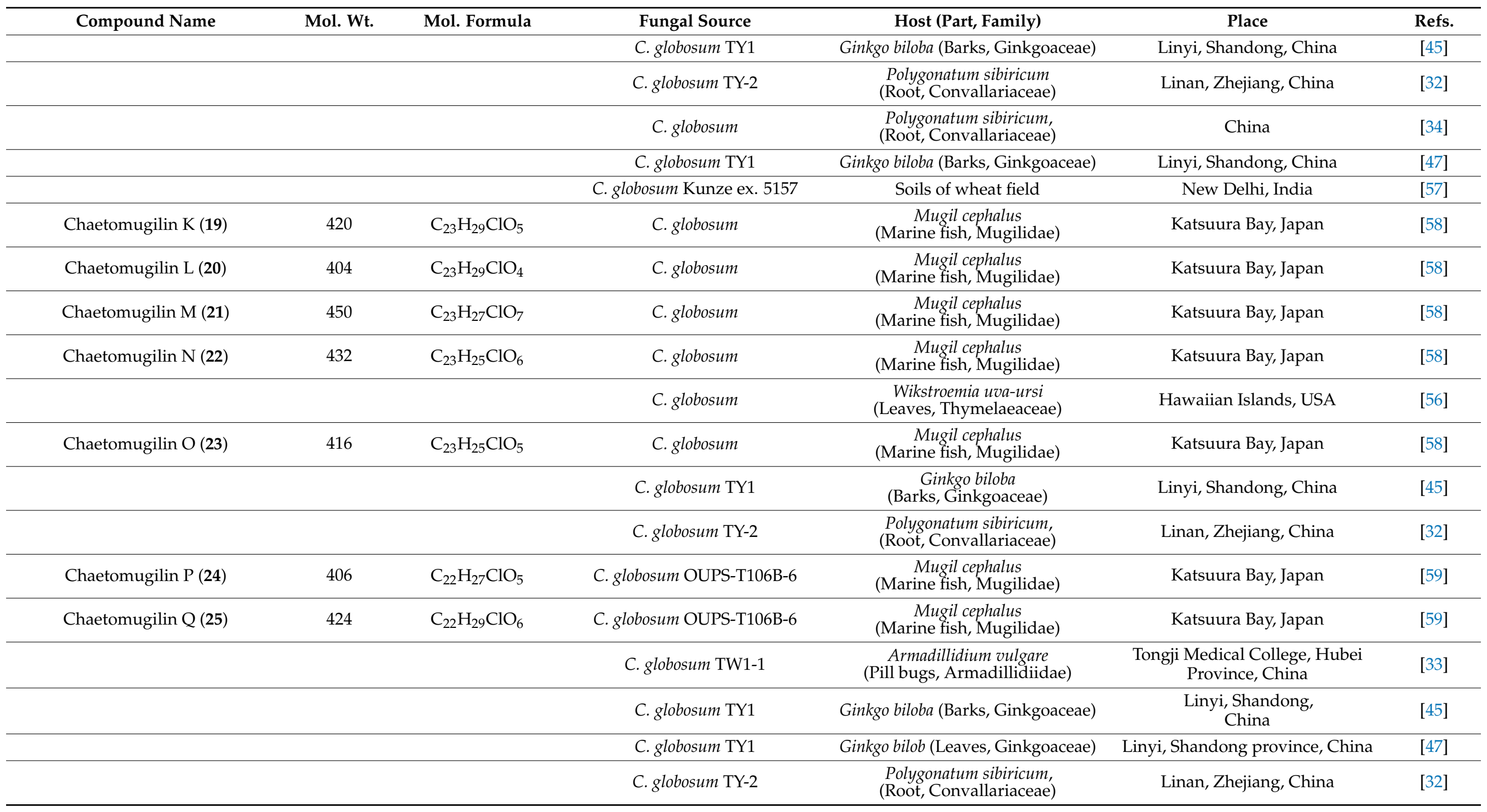


Table 1. Cont.

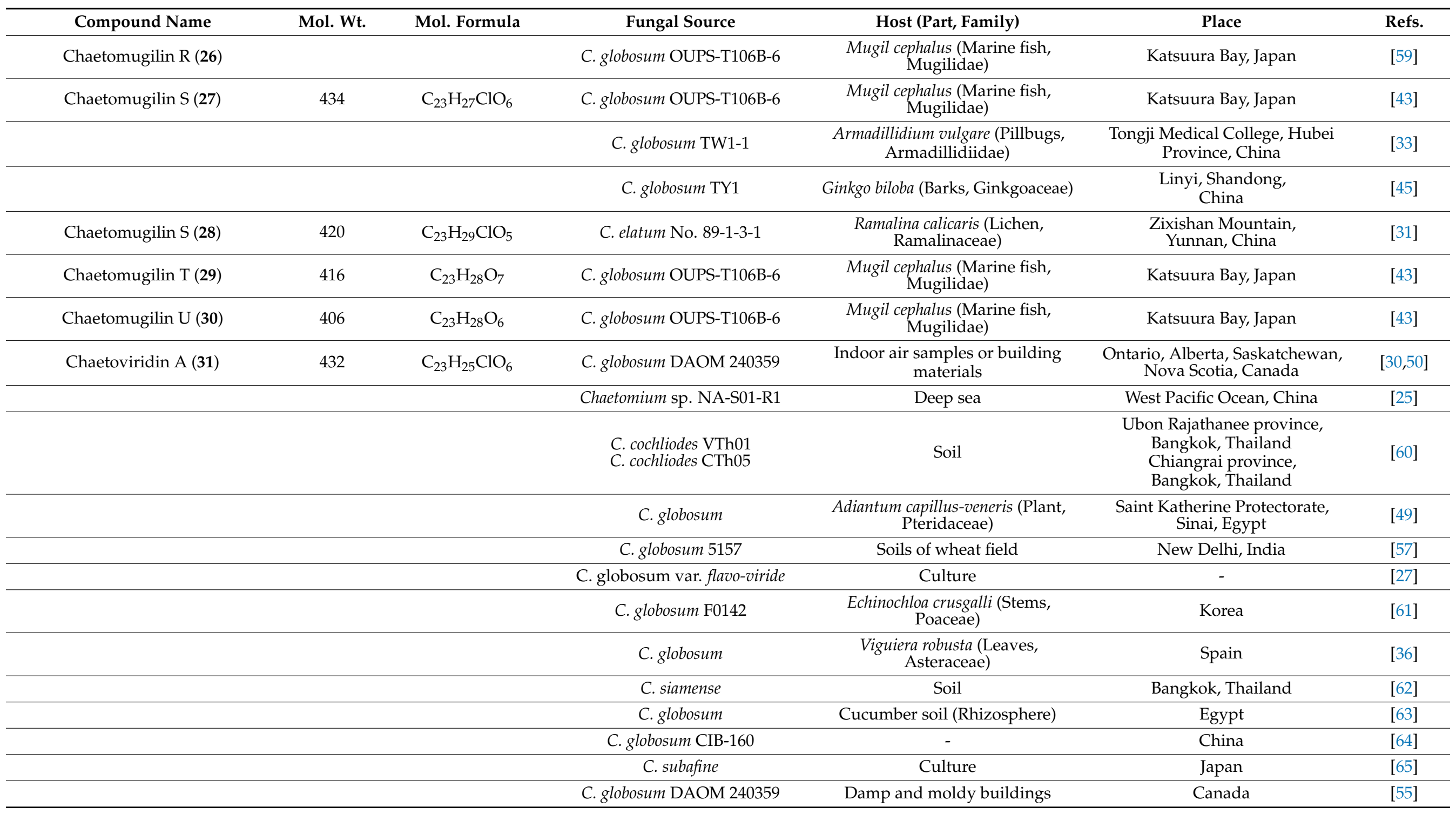


Table 1. Cont.

\begin{tabular}{|c|c|c|c|c|c|c|}
\hline Compound Name & Mol. Wt. & Mol. Formula & Fungal Source & Host (Part, Family) & Place & Refs. \\
\hline & & & C. globosum & Sea water and marine deposits & Jeju, Korea & [54] \\
\hline & & & C. globosum F211_UMNG & $\begin{array}{l}\text { Protium heptaphyllum } \\
\text { (Leaves, Burseraceae) }\end{array}$ & $\begin{array}{l}\text { Foothill of the west Colombian } \\
\text { Andes mountains, Aguazul, } \\
\text { Casanare, Colombia }\end{array}$ & {$[66]$} \\
\hline & & & C. globosum 22-10 & Soil & $\begin{array}{l}\text { PaLong ZangBu Brook, } \\
\text { Tibet, China }\end{array}$ & {$[67]$} \\
\hline & & & C. globosum & $\begin{array}{l}\text { Artemisia desterorum } \\
\text { (Roots, Asteraceae) }\end{array}$ & $\begin{array}{c}\text { Tengger Desert, } \\
\text { Ningxia Province, China }\end{array}$ & {$[68]$} \\
\hline & & & C. globosum CEF- 082 & $\begin{array}{l}\text { Gossypium arboreum } \\
\text { (Plant, Malvaceae) }\end{array}$ & China & {$[69]$} \\
\hline & & & C. globosum E-C-2 & $\begin{array}{l}\text { Apostichopus japonicas (Surface } \\
\text { muscle, Stichopodidae) }\end{array}$ & $\begin{array}{l}\text { Chengshantou Island, Weihai City, } \\
\text { the Yellow Sea, China }\end{array}$ & {$[70]$} \\
\hline & & & C. globosum & Indoor buildings & Finland & [53] \\
\hline & & & C. cochliodes & indoor buildings & Finland & [53] \\
\hline & & & C. globosum MP4-S01-7 & Sea water & West Pacific Ocean, China & [71] \\
\hline \multirow[t]{2}{*}{$4^{\prime}$-Epi-chaetoviridin A (32) } & 432 & $\mathrm{C}_{23} \mathrm{H}_{25} \mathrm{ClO}_{6}$ & C. globosum & $\begin{array}{c}\text { Viguiera robusta } \\
\text { (Leaves, Asteraceae) }\end{array}$ & Spain & {$[36]$} \\
\hline & & & C. globosum F211_UMNG & $\begin{array}{l}\text { Protium heptaphyllum } \\
\text { (Leaves, Burseraceae) }\end{array}$ & $\begin{array}{l}\text { Foothill of the west Colombian } \\
\text { Andes mountains, Aguazul, } \\
\text { Casanare, Colombia }\end{array}$ & {$[66]$} \\
\hline \multirow[t]{6}{*}{$5^{\prime}$-Epi-chaetoviridin A (33) } & 432 & $\mathrm{C}_{23} \mathrm{H}_{25} \mathrm{ClO}_{6}$ & $\begin{array}{l}\text { C. cochliodes VTh01 } \\
\text { C. cochliodes CTh05 }\end{array}$ & Soil & $\begin{array}{l}\text { Ubon Rajathanee province, } \\
\text { Bangkok, Thailand } \\
\text { Chiangrai province, } \\
\text { Bangkok, Thailand }\end{array}$ & {$[60]$} \\
\hline & & & C. globosum & $\begin{array}{c}\text { Viguiera robusta } \\
\text { (Leaves, Asteraceae) }\end{array}$ & Spain & {$[36]$} \\
\hline & & & C. globosum TY1 & Ginkgo biloba (Barks, Ginkgoaceae) & Linyi, Shandong province, China & {$[72]$} \\
\hline & & & C. globosum CDW7, & $\begin{array}{c}\text { Ginkgo biloba } \\
\text { (Leaves, Ginkgoaceae) }\end{array}$ & China & [73] \\
\hline & & & C. globosum 22-10 & Soil & $\begin{array}{c}\text { Palong Zangbu Brook, } \\
\text { Tibet, China }\end{array}$ & {$[67]$} \\
\hline & & & C. globosum F211_UMNG & $\begin{array}{l}\text { Protium heptaphyllum } \\
\text { (Leaves, Burseraceae) }\end{array}$ & $\begin{array}{l}\text { Foothill of the west Colombian } \\
\text { Andes mountains, Aguazul, } \\
\text { Casanare, Colombia }\end{array}$ & {$[66]$} \\
\hline
\end{tabular}


Table 1. Cont.

\begin{tabular}{|c|c|c|c|c|c|c|}
\hline Compound Name & Mol. Wt. & Mol. Formula & Fungal Source & Host (Part, Family) & Place & Refs. \\
\hline 7,5'-Bis-epi-chaetoviridin A (34) & 432 & $\mathrm{C}_{23} \mathrm{H}_{25} \mathrm{ClO}_{6}$ & C. elatum No. 89-1-3-1 & $\begin{array}{c}\text { Ramalina calicaris } \\
\text { (Lichen, Ramalinaceae) }\end{array}$ & $\begin{array}{l}\text { Zixishan Mountain, } \\
\text { Yunnan, China }\end{array}$ & {$[31]$} \\
\hline N-Glutarylchaetoviridin A (35) & 603 & $\mathrm{C}_{31} \mathrm{H}_{38} \mathrm{ClNO}_{9}$ & C. globosum HDN151398 & Sea sediment & South China Sea & [22] \\
\hline \multirow[t]{4}{*}{ Chaetoviridin B (37) } & 452 & $\mathrm{C}_{23} \mathrm{H}_{29} \mathrm{ClO}_{7}$ & C. globosum var. flavo-viride & Culture & - & [27] \\
\hline & & & C. globosum F0142 & $\begin{array}{l}\text { Echinochloa crusgalli } \\
\text { (Stems, Poaceae) }\end{array}$ & Korea & {$[61]$} \\
\hline & & & C. globosum & $\begin{array}{c}\text { Viguiera robusta } \\
\text { (Leaves, Asteraceae) }\end{array}$ & Spain & [36] \\
\hline & & & C. globosum & Cucumber soil (Rhizosphere) & Egypt & [63] \\
\hline \multirow[t]{6}{*}{ Chaetoviridin B (38) } & & & C. globosum 5157 & Soils of wheat field & New Delhi, India & [57] \\
\hline & & & C. globosum E-C-2 & $\begin{array}{c}\text { Apostichopus japonicas (Surface } \\
\text { muscle, Stichopodidae) }\end{array}$ & $\begin{array}{l}\text { Chengshantou Island, Weihai City, } \\
\text { the Yellow Sea, China }\end{array}$ & {$[70]$} \\
\hline & & & C. globosum Z1 & $\begin{array}{c}\text { Broussonetia papyrifera } \\
\text { (Barks, Moraceae) }\end{array}$ & Nanjing, Jiangsu, China & [44] \\
\hline & & & C. globosum TY1 & Ginkgo biloba (Barks, Ginkgoaceae) & Linyi, Shandong, China & [47] \\
\hline & & & C. globosum & $\begin{array}{l}\text { Adiantum capillus-veneris } \\
\text { (Plant, Pteridaceae) }\end{array}$ & $\begin{array}{c}\text { Saint Katherine Protectorate, } \\
\text { Sinai, Egypt }\end{array}$ & [49] \\
\hline & & & C. globosum TY1 & Ginkgo biloba (Barks, Ginkgoaceae) & Linyi, Shandong province, China & [72] \\
\hline N-Glutarylchaetoviridin B (39) & 543 & $\mathrm{C}_{28} \mathrm{H}_{30} \mathrm{ClNO}_{8}$ & C. globosum HDN151398 & Sea sediment & South China Sea & [22] \\
\hline \multirow[t]{4}{*}{ Chaetoviridin C (40) } & 434 & $\mathrm{C}_{23} \mathrm{H}_{27} \mathrm{ClO}_{6}$ & C. globosum var. flavo-viride & Culture & - & [27] \\
\hline & & & C. globosum & $\begin{array}{c}\text { Viguiera robusta } \\
\text { (Leaves, Asteraceae) }\end{array}$ & Spain & {$[36]$} \\
\hline & & & $\begin{array}{l}\text { Chaetomium globosum } \\
\text { OUPS-T106B-6 }\end{array}$ & $\begin{array}{c}\text { Mugil cephalus } \\
\text { (Marine fish, Mugilidae) }\end{array}$ & Katsuura Bay, Japan & [41] \\
\hline & & & C. globosum & Indoor buildings & Finland & [53] \\
\hline $12 \beta$-Hydroxychaetoviridin C (41) & 450 & $\mathrm{C}_{23} \mathrm{H}_{27} \mathrm{ClO}_{7}$ & C. globosum & $\begin{array}{c}\text { Viguiera robusta } \\
\text { (Leaves, Asteraceae) }\end{array}$ & Spain & [36] \\
\hline $\mathrm{N}$-Glutarylchaetoviridin C (42) & 571 & $\mathrm{C}_{30} \mathrm{H}_{34} \mathrm{ClNO}_{8}$ & C. globosum HDN151398 & Sea sediment & South China Sea & {$[22]$} \\
\hline
\end{tabular}


Table 1. Cont.

\begin{tabular}{|c|c|c|c|c|c|c|}
\hline Compound Name & Mol. Wt. & Mol. Formula & Fungal Source & Host (Part, Family) & Place & Refs. \\
\hline \multirow[t]{2}{*}{ Chaetoviridin D (43) } & 486 & $\mathrm{C}_{23} \mathrm{H}_{29} \mathrm{ClO}_{8}$ & C. globosum var. flavo-viride & Culture & Spain & [27] \\
\hline & & & C. globosum & $\begin{array}{c}\text { Viguiera robusta } \\
\text { (Leaves, Asteraceae) }\end{array}$ & Spain & [36] \\
\hline \multirow[t]{13}{*}{ Chaetoviridin E (44) } & 414 & $\mathrm{C}_{23} \mathrm{H}_{23} \mathrm{ClO}_{5}$ & C. globosum 5157 & Soils of wheat field & New Delhi, India & [57] \\
\hline & & & C. globosum MP4-S01-7 & Sea water & West Pacific Ocean, China & [71] \\
\hline & & & C. globosum & $\begin{array}{c}\text { Artemisia desterorum } \\
\text { (Roots, Asteraceae) }\end{array}$ & Tengger Desert in Ningxia, China. & [68] \\
\hline & & & C. globosum E-C-2 & $\begin{array}{c}\text { Apostichopus japonicas (Surface } \\
\text { muscle, Stichopodidae) }\end{array}$ & $\begin{array}{l}\text { Chengshantou Island, Weihai City, } \\
\text { the Yellow Sea, China }\end{array}$ & [70] \\
\hline & & & Chaetomium sp. NA-S01-R1 & Deep sea & West Pacific Ocean, China & [25] \\
\hline & & & C. globosum 22-10 & Soil & $\begin{array}{c}\text { PaLong ZangBu Brook, } \\
\text { Tibet, China }\end{array}$ & [67] \\
\hline & & & C. globosum & Sea water and marine deposits & Jeju, Korea & [54] \\
\hline & & & C. cochliodes CTh05 & Soil & $\begin{array}{c}\text { Ubon Rajathanee province, } \\
\text { Bangkok, Thailand }\end{array}$ & [60] \\
\hline & & & C. globosum & $\begin{array}{l}\text { Adiantum capillus-veneris } \\
\text { (Plant, Pteridaceae) }\end{array}$ & $\begin{array}{l}\text { Saint Katherine Protectorate, } \\
\text { Sinai, Egypt }\end{array}$ & [49] \\
\hline & & & C. globosum & $\begin{array}{c}\text { Viguiera robusta } \\
\text { (Leaves, Asteraceae) }\end{array}$ & Spain & [36] \\
\hline & & & Chaetomium siamense & Soil & Bangkok, Thailand & [62] \\
\hline & & & Chaetomium sp. & $\begin{array}{l}\text { Dromaius novaehollandiae } \\
\text { (Scat, Casuariidae) }\end{array}$ & Australia & [74] \\
\hline & & & C. globosum TY1 & Ginkgo biloba (Barks, Ginkgoaceae) & Linyi, Shandong province, China & [72] \\
\hline 7-Epi-chaetoviridin E (45) & 414 & $\mathrm{C}_{23} \mathrm{H}_{23} \mathrm{ClO}_{5}$ & C. elatum No. $89-1-3-1$ & $\begin{array}{c}\text { Ramalina calicaris } \\
\text { (Lichen, Ramalinaceae) }\end{array}$ & $\begin{array}{l}\text { Zixishan Mountain, } \\
\text { Yunnan, China }\end{array}$ & [31] \\
\hline $\begin{array}{c}\text { N-2-Butyric-azochaetoviridin E } \\
(\mathbf{4 6})\end{array}$ & 499 & $\mathrm{C}_{27} \mathrm{H}_{30} \mathrm{ClNO}_{6}$ & C. globosum DAOM 240359 & $\begin{array}{l}\text { Indoor air samples } \\
\text { or building materials }\end{array}$ & $\begin{array}{l}\text { Ontario, Alberta, Saskatchewan, } \\
\text { Nova Scotia, Canada }\end{array}$ & [50] \\
\hline Chaetoviridin F (47) & 416 & $\mathrm{C}_{23} \mathrm{H}_{25} \mathrm{ClO}_{5}$ & $\begin{array}{l}\text { C. cochliodes VTh01 } \\
\text { C. cochliodes CTh05 }\end{array}$ & Soil & $\begin{array}{l}\text { Ubon Rajathanee province, } \\
\text { Bangkok, Thailand } \\
\text { Chiangrai province, } \\
\text { Bangkok, Thailand }\end{array}$ & [60] \\
\hline
\end{tabular}


Table 1. Cont.

\begin{tabular}{|c|c|c|c|c|c|c|}
\hline Compound Name & Mol. Wt. & Mol. Formula & Fungal Source & Host (Part, Family) & Place & Refs. \\
\hline & & & C. globosum & $\begin{array}{c}\text { Viguiera robusta } \\
\text { (Leaves, Asteraceae) }\end{array}$ & Spain & [36] \\
\hline $4^{\prime}$-Epi-chaetoviridin F (48) & 416 & $\mathrm{C}_{23} \mathrm{H}_{25} \mathrm{ClO}_{5}$ & C. globosum & $\begin{array}{c}\text { Viguiera robusta } \\
\text { (Leaves, Asteraceae) }\end{array}$ & Spain & {$[36]$} \\
\hline Chaetoviridin G (49) & 416 & $\mathrm{C}_{23} \mathrm{H}_{25} \mathrm{ClO}_{5}$ & C. globosum & $\begin{array}{c}\text { Viguiera robusta } \\
\text { (Leaves, Asteraceae) }\end{array}$ & Spain & [36] \\
\hline Chaetoviridin G (50) & 420 & $\mathrm{C}_{23} \mathrm{H}_{29} \mathrm{ClO}_{5}$ & C. siamense & Soil & Bangkok, Thailand & [62] \\
\hline \multirow[t]{2}{*}{ Chaetoviridin H (51) } & 398 & $\mathrm{C}_{23} \mathrm{H}_{26} \mathrm{ClO}_{6}$ & C. globosum CBS148.51 & Cultured & China & [46] \\
\hline & & & C. globosum & $\begin{array}{c}\text { Viguiera robusta } \\
\text { (Leaves, Asteraceae) }\end{array}$ & Spain & [36] \\
\hline Chaetoviridin I (52) & 466 & $\mathrm{C}_{23} \mathrm{H}_{27} \mathrm{ClO}_{8}$ & C. globosum & $\begin{array}{c}\text { Viguiera robusta } \\
\text { (Leaves, Asteraceae) }\end{array}$ & Spain & {$[36]$} \\
\hline \multirow[t]{3}{*}{ Chaetoviridin J (53) } & 408 & $\mathrm{C}_{22} \mathrm{H}_{29} \mathrm{ClO}_{5}$ & C. globosum TY1 & Ginkgo biloba (Barks Ginkgoaceae) & Linyi, Shandong, China & [47] \\
\hline & & & C. globosum & $\begin{array}{c}\text { Wikstroemia uva-ursi } \\
\text { (Leaves, Thymelaeaceae) }\end{array}$ & Hawaiian Islands, USA & [56] \\
\hline & & & C. globosum & Seawater and marine deposits & Jeju, Korea & [54] \\
\hline Chaetoviridin K (54) & 450 & $\mathrm{C}_{23} \mathrm{H}_{27} \mathrm{ClO}_{7}$ & C. globosum & $\begin{array}{c}\text { Wikstroemia uva-ursi } \\
\text { (Leaves, Thymelaeaceae) }\end{array}$ & Hawaiian Islands, USA & [56] \\
\hline
\end{tabular}

Table 2. Biological activities of chaetomugilins and chaetoviridines.

\begin{tabular}{|c|c|c|c|c|c|}
\hline Compound Name & Biological Activity & Assay, Organism, or Cell Line & Biological Results & Positive Control & Refs. \\
\hline \multirow[t]{3}{*}{ Chaetomugilin 106B-6 XXVIII (1) } & Cytotoxicity & MTT/P388 & $32.0 \mu \mathrm{M}\left(\mathrm{IC}_{50}\right)$ & 5-FU $1.7 \mu \mathrm{M}\left(\mathrm{IC}_{50}\right)$ & {$[37,58]$} \\
\hline & & MTT/HL-60 & $51.8 \mu \mathrm{M}\left(\mathrm{IC}_{50}\right)$ & 5-FU $2.7 \mu \mathrm{M}\left(\mathrm{IC}_{50}\right)$ & {$[37,58]$} \\
\hline & & $\mathrm{MTT} / \mathrm{KB}$ & $58.8 \mu \mathrm{M}\left(\mathrm{IC}_{50}\right)$ & 5-FU $7.7 \mu \mathrm{M}\left(\mathrm{IC}_{50}\right)$ & {$[37,58]$} \\
\hline \multirow[t]{4}{*}{ Chaetomugilin A (2) } & Cytotoxicity & MTT/P388 & $8.7 \mu \mathrm{M}\left(\mathrm{IC}_{50}\right)$ & 5-FU $1.7 \mu \mathrm{M}\left(\mathrm{IC}_{50}\right)$ & {$[38,40]$} \\
\hline & & MTT/HL-60 & $7.3 \mu \mathrm{M}\left(\mathrm{IC}_{50}\right)$ & 5-FU $2.7 \mu \mathrm{M}\left(\mathrm{IC}_{50}\right)$ & {$[38,40]$} \\
\hline & & MTT/HL-60 & $6.4 \mu \mathrm{M}\left(\mathrm{IC}_{50}\right)$ & Adriamycin $0.1 \mu \mathrm{M}\left(\mathrm{IC}_{50}\right)$ & {$[22]$} \\
\hline & & MTT/K562 & $11.1 \mu \mathrm{M}\left(\mathrm{IC}_{50}\right)$ & Adriamycin $0.3 \mu \mathrm{M}\left(\mathrm{IC}_{50}\right)$ & {$[22]$} \\
\hline
\end{tabular}


Table 2. Cont.

\begin{tabular}{|c|c|c|c|c|c|}
\hline Compound Name & Biological Activity & Assay, Organism, or Cell Line & Biological Results & Positive Control & Refs. \\
\hline & & SRB/BEL-7402 & $17.9 \mu \mathrm{M}\left(\mathrm{IC}_{50}\right)$ & Adriamycin $0.4 \mu \mathrm{M}\left(\mathrm{IC}_{50}\right)$ & [22] \\
\hline & & SRB/HCT-116 & $6.1 \mu \mathrm{M}\left(\mathrm{IC}_{50}\right)$ & Adriamycin $0.2 \mu \mathrm{M}\left(\mathrm{IC}_{50}\right)$ & [22] \\
\hline & & $\mathrm{SRB} / \mathrm{HeLa}$ & $20.3 \mu \mathrm{M}\left(\mathrm{IC}_{50}\right)$ & Adriamycin $0.6 \mu \mathrm{M}\left(\mathrm{IC}_{50}\right)$ & [22] \\
\hline & & SRB/L-02 & $15.2 \mu \mathrm{M}\left(\mathrm{IC}_{50}\right)$ & Adriamycin $0.4 \mu \mathrm{M}\left(\mathrm{IC}_{50}\right)$ & [22] \\
\hline & & SRB/MGC-803 & $15.3 \mu \mathrm{M}\left(\mathrm{IC}_{50}\right)$ & Adriamycin $0.2 \mu \mathrm{M}\left(\mathrm{IC}_{50}\right)$ & [22] \\
\hline & & SRB/HO8910 & $12.1 \mu \mathrm{M}\left(\mathrm{IC}_{50}\right)$ & Adriamycin $0.4 \mu \mathrm{M}\left(\mathrm{IC}_{50}\right)$ & [22] \\
\hline & & SRB/SH-SY5Y & $23.4 \mu \mathrm{M}\left(\mathrm{IC}_{50}\right)$ & Adriamycin $0.2 \mu \mathrm{M}\left(\mathrm{IC}_{50}\right)$ & [22] \\
\hline & & SRB/NCI-H1975 & $18.3 \mu \mathrm{M}\left(\mathrm{IC}_{50}\right)$ & Adriamycin $0.3 \mu \mathrm{M}\left(\mathrm{IC}_{50}\right)$ & [22] \\
\hline & & SRB/U87 & $27.1 \mu \mathrm{M}\left(\mathrm{IC}_{50}\right)$ & Adriamycin $0.1 \mu \mathrm{M}\left(\mathrm{IC}_{50}\right)$ & [22] \\
\hline & & SRB/MDA-MB-231 & $22.7 \mu \mathrm{M}\left(\mathrm{IC}_{50}\right)$ & Adriamycin $0.2 \mu \mathrm{M}\left(\mathrm{IC}_{50}\right)$ & {$[22]$} \\
\hline \multirow[t]{4}{*}{ 11-Epi-chaetomugilin A (4) } & Cytotoxicity & MTT/P388 & $88.9 \mu \mathrm{M}\left(\mathrm{IC}_{50}\right)$ & 5-FU $1.7 \mu \mathrm{M}\left(\mathrm{IC}_{50}\right)$ & [35] \\
\hline & & MTT/HL-60 & $66.7 \mu \mathrm{M}\left(\mathrm{IC}_{50}\right)$ & 5-FU $2.7 \mu \mathrm{M}\left(\mathrm{IC}_{50}\right)$ & [35] \\
\hline & & MTT/P388 & $88.9 \mu \mathrm{M}\left(\mathrm{IC}_{50}\right)$ & 5-FU $1.7 \mu \mathrm{M}\left(\mathrm{IC}_{50}\right)$ & {$[37,58]$} \\
\hline & & MTT/L1210 & $80.2 \mu \mathrm{M}\left(\mathrm{IC}_{50}\right)$ & 5-FU $1.1 \mu \mathrm{M}\left(\mathrm{IC}_{50}\right)$ & {$[37,58]$} \\
\hline \multirow[t]{2}{*}{ Chaetomugilin B (6) } & Cytotoxicity & MTT/P388 & $18.7 \mu \mathrm{M}\left(\mathrm{IC}_{50}\right)$ & 5-FU $1.7 \mu \mathrm{M}\left(\mathrm{IC}_{50}\right)$ & {$[38,40]$} \\
\hline & & MTT/HL-60 & $16.5 \mu \mathrm{M}\left(\mathrm{IC}_{50}\right)$ & 5-FU $2.7 \mu \mathrm{M}\left(\mathrm{IC}_{50}\right)$ & {$[38,40]$} \\
\hline \multirow[t]{8}{*}{ Chaetomugilin C (7) } & Cytotoxicity & MTT/P388 & $3.6 \mu \mathrm{M}\left(\mathrm{IC}_{50}\right)$ & 5-FU $1.7 \mu \mathrm{M}\left(\mathrm{IC}_{50}\right)$ & {$[38,40]$} \\
\hline & & MTT/HL-60 & $2.7 \mu \mathrm{M}\left(\mathrm{IC}_{50}\right)$ & 5-FU $2.7 \mu \mathrm{M}\left(\mathrm{IC}_{50}\right)$ & {$[38,40]$} \\
\hline & & MTT/HL-60 & $6.6 \mu \mathrm{M}\left(\mathrm{IC}_{50}\right)$ & Adriamycin $0.1 \mu \mathrm{M}\left(\mathrm{IC}_{50}\right)$ & [22] \\
\hline & & MTT/K562 & $12.3 \mu \mathrm{M}\left(\mathrm{IC}_{50}\right)$ & Adriamycin $0.3 \mu \mathrm{M}\left(\mathrm{IC}_{50}\right)$ & [22] \\
\hline & & SRB/BEL-7402 & $16.8 \mu \mathrm{M}\left(\mathrm{IC}_{50}\right)$ & Adriamycin $0.4 \mu \mathrm{M}\left(\mathrm{IC}_{50}\right)$ & [22] \\
\hline & & SRB/HCT-116 & $5.7 \mu \mathrm{M}\left(\mathrm{IC}_{50}\right)$ & Adriamycin $0.2 \mu \mathrm{M}\left(\mathrm{IC}_{50}\right)$ & [22] \\
\hline & & $\mathrm{SRB} / \mathrm{HeLa}$ & $13.2 \mu \mathrm{M}\left(\mathrm{IC}_{50}\right)$ & Adriamycin $0.6 \mu \mathrm{M}\left(\mathrm{IC}_{50}\right)$ & [22] \\
\hline & & SRB/L-02 & $9.1 \mu \mathrm{M}\left(\mathrm{IC}_{50}\right)$ & Adriamycin $0.4 \mu \mathrm{M}\left(\mathrm{IC}_{50}\right)$ & {$[22]$} \\
\hline
\end{tabular}


Table 2. Cont.

\begin{tabular}{|c|c|c|c|c|c|}
\hline Compound Name & Biological Activity & Assay, Organism, or Cell Line & Biological Results & Positive Control & Refs. \\
\hline & & SRB/MGC-803 & $9.6 \mu \mathrm{M}\left(\mathrm{IC}_{50}\right)$ & Adriamycin $0.2 \mu \mathrm{M}\left(\mathrm{IC}_{50}\right)$ & [22] \\
\hline & & SRB/HO8910 & $8.8 \mu \mathrm{M}\left(\mathrm{IC}_{50}\right)$ & Adriamycin $0.4 \mu \mathrm{M}\left(\mathrm{IC}_{50}\right)$ & [22] \\
\hline & & SRB/SH-SY5Y & $19.4 \mu \mathrm{M}\left(\mathrm{IC}_{50}\right)$ & Adriamycin $0.2 \mu \mathrm{M}\left(\mathrm{IC}_{50}\right)$ & [22] \\
\hline & & SRB/NCI-H1975 & $12.1 \mu \mathrm{M}\left(\mathrm{IC}_{50}\right)$ & Adriamycin $0.3 \mu \mathrm{M}\left(\mathrm{IC}_{50}\right)$ & [22] \\
\hline & & SRB/U87 & $17.6 \mu \mathrm{M}\left(\mathrm{IC}_{50}\right)$ & Adriamycin $0.1 \mu \mathrm{M}\left(\mathrm{IC}_{50}\right)$ & [22] \\
\hline & & SRB/MDA-MB-231 & $26.6 \mu \mathrm{M}\left(\mathrm{IC}_{50}\right)$ & Adriamycin $0.2 \mu \mathrm{M}\left(\mathrm{IC}_{50}\right)$ & [22] \\
\hline \multirow{6}{*}{ Chaetomugilin D (8) } & & MTT/HL-60 & $6.8 \mu \mathrm{M}\left(\mathrm{IC}_{50}\right)$ & 5-FU $2.7 \mu \mathrm{M}\left(\mathrm{IC}_{50}\right)$ & {$[38,40]$} \\
\hline & Phytotoxic activity & $\begin{array}{l}\text { Lettuce seed germination } \\
\text { bioassay/Root growth inhibition }\end{array}$ & $24.2 \mathrm{ppm}\left(\mathrm{IC}_{50}\right)$ & - & [51] \\
\hline & & $\begin{array}{l}\text { Lettuce seed germination } \\
\text { bioassay/Shoot growth inhibition }\end{array}$ & $27.8 \mathrm{ppm}\left(\mathrm{IC}_{50}\right)$ & - & [51] \\
\hline & Antimicrobial & Microplate assay/Vibrio vulnificus & $32.4 \mu \mathrm{g} / \mathrm{mL}(\mathrm{MIC})$ & Erythromycin $2.0 \mu \mathrm{g} / \mathrm{mL}$ (MIC) & [25] \\
\hline & & Microplate assay/MRSA 1 & $32.2 \mu \mathrm{g} / \mathrm{mL}(\mathrm{MIC})$ & $\begin{array}{l}\text { Chloramphenicol } \\
7.6 \mu \mathrm{g} / \mathrm{mL} \text { (MIC) }\end{array}$ & [25] \\
\hline & & Microplate assay/MRSA 2 & 32.4 g /mL (MIC) & $\begin{array}{l}\text { Chloramphenicol } \\
7.5 \mu \mathrm{g} / \mathrm{mL} \text { (MIC) }\end{array}$ & [25] \\
\hline \multirow[t]{4}{*}{ Seco-chaetomugilin D (9) } & Cytotoxicity & MTT/P388 & $38.6 \mu \mathrm{M}\left(\mathrm{IC}_{50}\right)$ & 5-FU $1.7 \mu \mathrm{M}\left(\mathrm{IC}_{50}\right)$ & [48] \\
\hline & & MTT/HL-60 & $47.2 \mu \mathrm{M}\left(\mathrm{IC}_{50}\right)$ & 5-FU $2.7 \mu \mathrm{M}\left(\mathrm{IC}_{50}\right)$ & [48] \\
\hline & & MTT/L1210 & $53.6 \mu \mathrm{M}\left(\mathrm{IC}_{50}\right)$ & 5-FU $3.0 \mu \mathrm{M}\left(\mathrm{IC}_{50}\right)$ & [48] \\
\hline & & $\mathrm{MTT} / \mathrm{KB}$ & $47.2 \mu \mathrm{M}\left(\mathrm{IC}_{50}\right)$ & 5-FU $6.0 \mu \mathrm{M}\left(\mathrm{IC}_{50}\right)$ & [48] \\
\hline \multirow[t]{4}{*}{ Chaetomugilin E (11) } & Cytotoxicity & MTT/P388 & $15.7 \mu \mathrm{M}\left(\mathrm{IC}_{50}\right)$ & 5-FU $1.7 \mu \mathrm{M}\left(\mathrm{IC}_{50}\right)$ & {$[38,40]$} \\
\hline & & MTT/HL-60 & $13.2 \mu \mathrm{M}\left(\mathrm{IC}_{50}\right)$ & 5-FU $2.7 \mu \mathrm{M}\left(\mathrm{IC}_{50}\right)$ & {$[38,40]$} \\
\hline & Inhibition TNF- $\alpha$ & TNF- $\alpha$ activated NF-kB assay & $11.6 \mu \mathrm{M}\left(\mathrm{IC}_{50}\right)$ & $\begin{array}{l}\text { TPCK } 3.8 \mu \mathrm{M}\left(\mathrm{IC}_{50}\right) \\
\text { BAY-11 } 2.0 \mu \mathrm{M}\left(\mathrm{IC}_{50}\right)\end{array}$ & {$[56]$} \\
\hline & Inhibition NO & Nitrite assay & $5.8 \mu \mathrm{M}\left(\mathrm{IC}_{50}\right)$ & L-NMMA $25.1 \mu \mathrm{M}\left(\mathrm{IC}_{50}\right)$ & [56] \\
\hline
\end{tabular}


Table 2. Cont.

\begin{tabular}{|c|c|c|c|c|c|}
\hline Compound Name & Biological Activity & Assay, Organism, or Cell Line & Biological Results & Positive Control & Refs. \\
\hline \multirow[t]{4}{*}{ Chaetomugilin F (13) } & Cytotoxicity & MTT/P388 & $3.3 \mu \mathrm{M}\left(\mathrm{IC}_{50}\right)$ & 5-FU $1.7 \mu \mathrm{M}\left(\mathrm{IC}_{50}\right)$ & {$[38,40]$} \\
\hline & & MTT/HL-60 & $1.3 \mu \mathrm{M}\left(\mathrm{IC}_{50}\right)$ & 5-FU $2.7 \mu \mathrm{M}\left(\mathrm{IC}_{50}\right)$ & {$[38,40]$} \\
\hline & Inhibition TNF- $\alpha$ & TNF- $\alpha$ activated NF-kB assay & $5.1 \mu \mathrm{M}\left(\mathrm{IC}_{50}\right)$ & $\begin{array}{l}\text { TPCK } 3.8 \mu \mathrm{M}\left(\mathrm{IC}_{50}\right) \\
\text { BAY-11, } 2.0 \mu \mathrm{M}\left(\mathrm{IC}_{50}\right)\end{array}$ & [56] \\
\hline & Inhibition NO & Nitrite assay & $1.9 \mu \mathrm{M}\left(\mathrm{IC}_{50}\right)$ & L-NMMA $25.1 \mu \mathrm{M}\left(\mathrm{IC}_{50}\right)$ & [56] \\
\hline \multirow[t]{5}{*}{ Chaetomugilin G (14) } & Cytotoxicity & MTT/P388 & $24.1 \mu \mathrm{M}\left(\mathrm{IC}_{50}\right)$ & 5-FU $1.7 \mu \mathrm{M}\left(\mathrm{IC}_{50}\right)$ & [38] \\
\hline & & MTT/P388 & $24.1 \mu \mathrm{M}\left(\mathrm{IC}_{50}\right)$ & 5-FU $1.7 \mu \mathrm{M}\left(\mathrm{IC}_{50}\right)$ & [41] \\
\hline & & MTT/HL-60 & $19.8 \mu \mathrm{M}\left(\mathrm{IC}_{50}\right)$ & 5-FU $2.7 \mu \mathrm{M}\left(\mathrm{IC}_{50}\right)$ & [41] \\
\hline & & MTT/L1210 & $123.6 \mu \mathrm{M}\left(\mathrm{IC}_{50}\right)$ & 5-FU $3.0 \mu \mathrm{M}\left(\mathrm{IC}_{50}\right)$ & [41] \\
\hline & & $\mathrm{MTT} / \mathrm{KB}$ & $137.8 \mu \mathrm{M}\left(\mathrm{IC}_{50}\right)$ & 5-FU $6.0 \mu \mathrm{M}\left(\mathrm{IC}_{50}\right)$ & [41] \\
\hline \multirow[t]{5}{*}{ Chaetomugilin H (15) } & Cytotoxicity & MTT/P388 & $12.3 \mu \mathrm{M}\left(\mathrm{IC}_{50}\right)$ & 5-FU $1.7 \mu \mathrm{M}\left(\mathrm{IC}_{50}\right)$ & [38] \\
\hline & & MTT/HL-60 & $10.3 \mu \mathrm{M}\left(\mathrm{IC}_{50}\right)$ & 5-FU $2.7 \mu \mathrm{M}\left(\mathrm{IC}_{50}\right)$ & [38] \\
\hline & & MTT/HL-60 & $10.3 \mu \mathrm{M}\left(\mathrm{IC}_{50}\right)$ & 5-FU $2.7 \mu \mathrm{M}\left(\mathrm{IC}_{50}\right)$ & [41] \\
\hline & & MTT/L1210 & $93.3 \mu \mathrm{M}\left(\mathrm{IC}_{50}\right)$ & 5-FU $3.0 \mu \mathrm{M}\left(\mathrm{IC}_{50}\right)$ & [41] \\
\hline & & $\mathrm{MTT} / \mathrm{KB}$ & $18.8 \mu \mathrm{M}\left(\mathrm{IC}_{50}\right)$ & 5-FU $6.0 \mu \mathrm{M}\left(\mathrm{IC}_{50}\right)$ & [41] \\
\hline \multirow[t]{6}{*}{ Chaetomugilin I (16) } & Cytotoxicity & MTT/P388 & $1.1 \mu \mathrm{M}\left(\mathrm{IC}_{50}\right)$ & 5-FU $1.7 \mu \mathrm{M}\left(\mathrm{IC}_{50}\right)$ & {$[37,58,59]$} \\
\hline & & MTT/HL-60 & $1.1 \mu \mathrm{M}\left(\mathrm{IC}_{50}\right)$ & 5-FU $2.7 \mu \mathrm{M}\left(\mathrm{IC}_{50}\right)$ & {$[37,58,59]$} \\
\hline & & MTT/L1210 & $1.9 \mu \mathrm{M}\left(\mathrm{IC}_{50}\right)$ & 5-FU $1.1 \mu \mathrm{M}\left(\mathrm{IC}_{50}\right)$ & {$[37,58,59]$} \\
\hline & & $\mathrm{MTT} / \mathrm{KB}$ & $2.3 \mu \mathrm{M}\left(\mathrm{IC}_{50}\right)$ & 5-FU $7.7 \mu \mathrm{M}\left(\mathrm{IC}_{50}\right)$ & {$[37,58,59]$} \\
\hline & Inhibition TNF- $\alpha$ & TNF- $\alpha$ activated NF-kB assay & $0.9 \mu \mathrm{M}\left(\mathrm{IC}_{50}\right)$ & $\begin{array}{l}\text { TPCK } 3.8 \mu \mathrm{M}\left(\mathrm{IC}_{50}\right) \\
\text { BAY-11 } 2.0 \mu \mathrm{M}\left(\mathrm{IC}_{50}\right)\end{array}$ & [56] \\
\hline & Inhibition NO & Nitrite assay & $0.3 \mu \mathrm{M}\left(\mathrm{IC}_{50}\right)$ & L-NMMA $25.1 \mu \mathrm{M}\left(\mathrm{IC}_{50}\right)$ & [56] \\
\hline \multirow[t]{2}{*}{ 11-Epi-chaetomugilin I (17) } & Cytotoxicity & MTT/P388 & $0.7 \mu \mathrm{M}\left(\mathrm{IC}_{50}\right)$ & 5-FU $1.7 \mu \mathrm{M}\left(\mathrm{IC}_{50}\right)$ & [59] \\
\hline & & MTT/HL-60 & $1.0 \mu \mathrm{M}\left(\mathrm{IC}_{50}\right)$ & 5-FU $2.7 \mu \mathrm{M}\left(\mathrm{IC}_{50}\right)$ & [59] \\
\hline
\end{tabular}


Table 2. Cont.

\begin{tabular}{|c|c|c|c|c|c|}
\hline Compound Name & Biological Activity & Assay, Organism, or Cell Line & Biological Results & Positive Control & Refs. \\
\hline & & MTT/L1210 & $1.6 \mu \mathrm{M}\left(\mathrm{IC}_{50}\right)$ & 5-FU $1.1 \mu \mathrm{M}\left(\mathrm{IC}_{50}\right)$ & [59] \\
\hline & & $\mathrm{MTT} / \mathrm{KB}$ & $1.2 \mu \mathrm{M}\left(\mathrm{IC}_{50}\right)$ & 5-FU $7.7 \mu \mathrm{M}\left(\mathrm{IC}_{50}\right)$ & [59] \\
\hline & Inhibition TNF- $\alpha$ & TNF- $\alpha$ activated NF-kB assay & $0.9 \mu \mathrm{M}\left(\mathrm{IC}_{50}\right)$ & $\begin{array}{l}\text { TPCK } 3.8 \mu \mathrm{M}\left(\mathrm{IC}_{50}\right) \\
\text { BAY-11 } 2.0 \mu \mathrm{M}\left(\mathrm{IC}_{50}\right)\end{array}$ & {$[56]$} \\
\hline & Inhibition NO & Nitrite assay & $0.8 \mu \mathrm{M}\left(\mathrm{IC}_{50}\right)$ & L-NMMA $25.1 \mu \mathrm{M}\left(\mathrm{IC}_{50}\right)$ & {$[56]$} \\
\hline \multirow[t]{8}{*}{ Chaetomugilin J (18) } & Phytotoxic activity & $\begin{array}{l}\text { Lettuce seed germination } \\
\text { bioassay/Root growth inhibition }\end{array}$ & $22.6 \mathrm{ppm}\left(\mathrm{IC}_{50}\right)$ & - & {$[51]$} \\
\hline & & $\begin{array}{l}\text { Lettuce seed germination } \\
\text { bioassay/Shoot growth inhibition }\end{array}$ & $21.9 \mathrm{ppm}\left(\mathrm{IC}_{50}\right)$ & - & {$[51]$} \\
\hline & Cytotoxicity & MTT/P388 & $12.6 \mu \mathrm{M}\left(\mathrm{IC}_{50}\right)$ & 5-FU $1.7 \mu \mathrm{M}\left(\mathrm{IC}_{50}\right)$ & {$[37,58$} \\
\hline & & MTT/HL-60 & $12.6 \mu \mathrm{M}\left(\mathrm{IC}_{50}\right)$ & 5-FU $2.7 \mu \mathrm{M}\left(\mathrm{IC}_{50}\right)$ & {$[37,58]$} \\
\hline & & MTT/L1210 & $2.8 \mu \mathrm{M}\left(\mathrm{IC}_{50}\right)$ & 5-FU $1.1 \mu \mathrm{M}\left(\mathrm{IC}_{50}\right)$ & {$[37,58]$} \\
\hline & & $\mathrm{MTT} / \mathrm{KB}$ & $8.5 \mu \mathrm{M}\left(\mathrm{IC}_{50}\right)$ & 5-FU $7.7 \mu \mathrm{M}\left(\mathrm{IC}_{50}\right)$ & {$[37,58]$} \\
\hline & Inhibition TNF- $\alpha$ & TNF- $\alpha$ activated NF-kB assay & $7.6 \mu \mathrm{M}\left(\mathrm{IC}_{50}\right)$ & $\begin{array}{l}\text { TPCK } 3.8 \mu \mathrm{M}\left(\mathrm{IC}_{50}\right) \\
\text { BAY-11 } 2.0 \mu \mathrm{M}\left(\mathrm{IC}_{50}\right)\end{array}$ & [56] \\
\hline & Inhibition NO & Nitrite assay & $4.2 \mu \mathrm{M}\left(\mathrm{IC}_{50}\right)$ & L-NMMA $25.1 \mu \mathrm{M}\left(\mathrm{IC}_{50}\right)$ & [56] \\
\hline \multirow[t]{4}{*}{ Chaetomugilin K (19) } & Cytotoxicity & MTT/P388 & $8.2 \mu \mathrm{M}\left(\mathrm{IC}_{50}\right)$ & 5-FU $1.7 \mu \mathrm{M}\left(\mathrm{IC}_{50}\right)$ & [58] \\
\hline & & MTT/HL-60 & $14.1 \mu \mathrm{M}\left(\mathrm{IC}_{50}\right)$ & 5-FU $2.7 \mu \mathrm{M}\left(\mathrm{IC}_{50}\right)$ & [58] \\
\hline & & MTT/L1210 & $11.2 \mu \mathrm{M}\left(\mathrm{IC}_{50}\right)$ & 5-FU $1.1 \mu \mathrm{M}\left(\mathrm{IC}_{50}\right)$ & [58] \\
\hline & & $\mathrm{MTT} / \mathrm{KB}$ & $18.7 \mu \mathrm{M}\left(\mathrm{IC}_{50}\right)$ & 5-FU $7.7 \mu \mathrm{M}\left(\mathrm{IC}_{50}\right)$ & [58] \\
\hline \multirow[t]{4}{*}{ Chaetomugilin L (20) } & Cytotoxicity & MTT/P388 & $10.9 \mu \mathrm{M}\left(\mathrm{IC}_{50}\right)$ & 5-FU $1.7 \mu \mathrm{M}\left(\mathrm{IC}_{50}\right)$ & [58] \\
\hline & & MTT/HL-60 & $13.1 \mu \mathrm{M}\left(\mathrm{IC}_{50}\right)$ & 5-FU $2.7 \mu \mathrm{M}\left(\mathrm{IC}_{50}\right)$ & [58] \\
\hline & & MTT/L1210 & $15.6 \mu \mathrm{M}\left(\mathrm{IC}_{50}\right)$ & 5-FU $1.1 \mu \mathrm{M}\left(\mathrm{IC}_{50}\right)$ & {$[58]$} \\
\hline & & $\mathrm{MTT} / \mathrm{KB}$ & $20.1 \mu \mathrm{M}\left(\mathrm{IC}_{50}\right)$ & 5-FU $7.7 \mu \mathrm{M}\left(\mathrm{IC}_{50}\right)$ & [58] \\
\hline \multirow[t]{3}{*}{ Chaetomugilin N (22) } & Cytotoxicity & MTT/P388 & $2.3 \mu \mathrm{M}\left(\mathrm{IC}_{50}\right)$ & 5-FU $1.7 \mu \mathrm{M}\left(\mathrm{IC}_{50}\right)$ & {$[58]$} \\
\hline & & MTT/HL-60 & $2.3 \mu \mathrm{M}\left(\mathrm{IC}_{50}\right)$ & 5-FU $2.7 \mu \mathrm{M}\left(\mathrm{IC}_{50}\right)$ & [58] \\
\hline & & MTT/L1210 & $10.6 \mu \mathrm{M}\left(\mathrm{IC}_{50}\right)$ & 5-FU $1.1 \mu \mathrm{M}\left(\mathrm{IC}_{50}\right)$ & {$[58]$} \\
\hline
\end{tabular}


Table 2. Cont.

\begin{tabular}{|c|c|c|c|c|c|}
\hline Compound Name & Biological Activity & Assay, Organism, or Cell Line & Biological Results & Positive Control & Refs. \\
\hline & & $\mathrm{MTT} / \mathrm{KB}$ & $10.6 \mu \mathrm{M}\left(\mathrm{IC}_{50}\right)$ & 5-FU $7.7 \mu \mathrm{M}\left(\mathrm{IC}_{50}\right)$ & [58] \\
\hline \multirow[t]{4}{*}{ Chaetomugilin O (23) } & Cytotoxicity & MTT/P388 & $11.1 \mu \mathrm{M}\left(\mathrm{IC}_{50}\right)$ & 5-FU $1.7 \mu \mathrm{M}\left(\mathrm{IC}_{50}\right)$ & [58] \\
\hline & & MTT/HL-60 & $11.1 \mu \mathrm{M}\left(\mathrm{IC}_{50}\right)$ & 5-FU $2.7 \mu \mathrm{M}\left(\mathrm{IC}_{50}\right)$ & [58] \\
\hline & & MTT/L1210 & $10.1 \mu \mathrm{M}\left(\mathrm{IC}_{50}\right)$ & 5-FU $1.1 \mu \mathrm{M}\left(\mathrm{IC}_{50}\right)$ & [58] \\
\hline & & MTT/KB & $7.2 \mu \mathrm{M}\left(\mathrm{IC}_{50}\right)$ & 5-FU $7.7 \mu \mathrm{M}\left(\mathrm{IC}_{50}\right)$ & [58] \\
\hline \multirow[t]{4}{*}{ Chaetomugilin P (24) } & Cytotoxicity & MTT/P388 & $0.7 \mu \mathrm{M}\left(\mathrm{IC}_{50}\right)$ & 5-FU $1.7 \mu \mathrm{M}\left(\mathrm{IC}_{50}\right)$ & [59] \\
\hline & & MTT/HL-60 & $1.2 \mu \mathrm{M}\left(\mathrm{IC}_{50}\right)$ & 5-FU $2.7 \mu \mathrm{M}\left(\mathrm{IC}_{50}\right)$ & [59] \\
\hline & & MTT/L1210 & $1.5 \mu \mathrm{M}\left(\mathrm{IC}_{50}\right)$ & 5-FU $1.1 \mu \mathrm{M}\left(\mathrm{IC}_{50}\right)$ & [59] \\
\hline & & $\mathrm{MTT} / \mathrm{KB}$ & $1.8 \mu \mathrm{M}\left(\mathrm{IC}_{50}\right)$ & 5-FU $7.7 \mu \mathrm{M}\left(\mathrm{IC}_{50}\right)$ & [59] \\
\hline \multirow[t]{3}{*}{ Chaetomugilin Q (25) } & Cytotoxicity & MTT/P388 & $49.5 \mu \mathrm{M}\left(\mathrm{IC}_{50}\right)$ & 5-FU $1.7 \mu \mathrm{M}\left(\mathrm{IC}_{50}\right)$ & [59] \\
\hline & & MTT/HL-60 & $47.2 \mu \mathrm{M}\left(\mathrm{IC}_{50}\right)$ & 5-FU $2.7 \mu \mathrm{M}\left(\mathrm{IC}_{50}\right)$ & [59] \\
\hline & & MTT/L1210 & $80.2 \mu \mathrm{M}\left(\mathrm{IC}_{50}\right)$ & 5-FU $1.1 \mu \mathrm{M}\left(\mathrm{IC}_{50}\right)$ & [59] \\
\hline \multirow[t]{3}{*}{ Chaetomugilin R (26) } & Cytotoxicity & MTT/P388 & $32.0 \mu \mathrm{M}\left(\mathrm{IC}_{50}\right)$ & 5-FU $1.7 \mu \mathrm{M}\left(\mathrm{IC}_{50}\right)$ & [59] \\
\hline & & MTT/L1210 & $67.1 \mu \mathrm{M}\left(\mathrm{IC}_{50}\right)$ & 5-FU $1.1 \mu \mathrm{M}\left(\mathrm{IC}_{50}\right)$ & [59] \\
\hline & & MTT/KB & $67.1 \mu \mathrm{M}\left(\mathrm{IC}_{50}\right)$ & 5-FU $7.7 \mu \mathrm{M}\left(\mathrm{IC}_{50}\right)$ & [59] \\
\hline Chaetomugilin S (27) & Caspase-3 inhibitory & Caspase- 3 enzymatic assay & $20.6 \mu \mathrm{M}\left(\mathrm{IC}_{50}\right)$ & Ac-DEVD-CHO $13.7 \mathrm{nM}\left(\mathrm{IC}_{50}\right)$ & [31] \\
\hline \multirow[t]{2}{*}{ Chaetomugilin T (29) } & Cytotoxicity & MTT/P388 & $62.4 \mu \mathrm{M}\left(\mathrm{IC}_{50}\right)$ & 5-FU $1.9 \mu \mathrm{M}\left(\mathrm{IC}_{50}\right)$ & [43] \\
\hline & & MTT/HL-60 & $67.2 \mu \mathrm{M}\left(\mathrm{IC}_{50}\right)$ & 5-FU $2.3 \mu \mathrm{M}\left(\mathrm{IC}_{50}\right)$ & [43] \\
\hline \multirow[t]{3}{*}{ Chaetomugilin U (30) } & Cytotoxicity & MTT/P388 & $57.4 \mu \mathrm{M}\left(\mathrm{IC}_{50}\right)$ & 5-FU $1.9 \mu \mathrm{M}\left(\mathrm{IC}_{50}\right)$ & [43] \\
\hline & & MTT/HL-60 & $57.4 \mu \mathrm{M}\left(\mathrm{IC}_{50}\right)$ & 5-FU $2.3 \mu \mathrm{M}\left(\mathrm{IC}_{50}\right)$ & [43] \\
\hline & & MTT/L1210 & $94.8 \mu \mathrm{M}\left(\mathrm{IC}_{50}\right)$ & 5-FU $2.2 \mu \mathrm{M}\left(\mathrm{IC}_{50}\right)$ & [43] \\
\hline \multirow[t]{4}{*}{ Chaetoviridin A (31) } & Antifungal & Alternaria mali & $33.3 \mu \mathrm{g} / \mathrm{mL}$ (MIC) & - & [61] \\
\hline & & Botrytis cinerea & $33.3 \mu \mathrm{g} / \mathrm{mL}$ (MIC) & - & [61] \\
\hline & & Colletotrichum gloeosporioides & $33.3 \mu \mathrm{g} / \mathrm{mL}$ (MIC) & - & [61] \\
\hline & & Fusarium oxysporum & $33.3 \mu \mathrm{g} / \mathrm{mL}(\mathrm{MIC})$ & - & {$[61]$} \\
\hline
\end{tabular}


Table 2. Cont.

\begin{tabular}{|c|c|c|c|c|c|}
\hline Compound Name & Biological Activity & Assay, Organism, or Cell Line & Biological Results & Positive Control & Refs. \\
\hline & & Phytophthora capsici & $33.3 \mu \mathrm{g} / \mathrm{mL}(\mathrm{MIC})$ & - & {$[61]$} \\
\hline & & Phytophthora infestans & $33.3 \mu \mathrm{g} / \mathrm{mL}$ (MIC) & - & [61] \\
\hline & & Pythium ultimum & $1.23 \mu \mathrm{g} / \mathrm{mL}(\mathrm{MIC})$ & - & [61] \\
\hline & & Magnaporthe grisea & $1.23 \mu \mathrm{g} / \mathrm{mL}(\mathrm{MIC})$ & - & [61] \\
\hline & & Sclerotinia sclerotiorum & 97.5 (\% inhibition) & - & [73] \\
\hline & & Botrytis cinerea & 69.1 (\% inhibition) & - & [73] \\
\hline & & Fusarium graminearum & 77.0 (\% inhibition) & - & [73] \\
\hline & & Phytophthora capsici & 60.7 (\% inhibition) & - & [73] \\
\hline & & Fusarium moniliforme & 59.2 (\% inhibition) & - & [73] \\
\hline 5'-Epi-chaetoviridin A (33) & Cytotoxicity & SRB assay/HepG-2 & $35.3 \mu \mathrm{M}\left(\mathrm{IC}_{50}\right)$ & Camptothecin $32.3 \mu \mathrm{M}\left(\mathrm{IC}_{50}\right)$ & {$[72]$} \\
\hline 7,5'-Bis-epi-chaetoviridin A (34) & Caspase-3 inhibitory & Caspase-3 enzymatic assay & $10.9 \mu \mathrm{M}\left(\mathrm{IC}_{50}\right)$ & Ac-DEVD-CHO 13.7 nM $\left(\mathrm{IC}_{50}\right)$ & [31] \\
\hline \multirow[t]{2}{*}{ N-glutarylchaetoviridin A (35) } & Cytotoxicity & MTT/HL-60 & $10.3 \mu \mathrm{M}\left(\mathrm{IC}_{50}\right)$ & Adriamycin $0.1 \mu \mathrm{M}\left(\mathrm{IC}_{50}\right)$ & [22] \\
\hline & & MTT/K562 & $20.3 \mu \mathrm{M}\left(\mathrm{IC}_{50}\right)$ & Adriamycin $0.3 \mu \mathrm{M}\left(\mathrm{IC}_{50}\right)$ & {$[22]$} \\
\hline \multirow[t]{2}{*}{ Chaetoviridin B (37) } & Antifungal & Pythium ultimum & 33.3 g /mL (MIC) & - & {$[61]$} \\
\hline & & Magnaporthe grisea & $33.3 \mu \mathrm{g} / \mathrm{mL}(\mathrm{MIC})$ & - & [61] \\
\hline Chaetoviridin B (38) & $\alpha$-Glucosidase inhibiory & Spectrophotometric assay & $6.328 \mu \mathrm{M}\left(\mathrm{IC}_{50}\right)$ & Acarbose $54.74 \mu \mathrm{M}\left(\mathrm{IC}_{50}\right)$ & [47] \\
\hline \multirow[t]{8}{*}{$\mathrm{N}$-Glutarylchaetoviridin C (42) } & Cytotoxicity & MTT/HL-60 & $11.1 \mu \mathrm{M}\left(\mathrm{IC}_{50}\right)$ & Adriamycin $0.1 \mu \mathrm{M}\left(\mathrm{IC}_{50}\right)$ & {$[22]$} \\
\hline & & MTT/K562 & $11.7 \mu \mathrm{M}\left(\mathrm{IC}_{50}\right)$ & Adriamycin $0.3 \mu \mathrm{M}\left(\mathrm{IC}_{50}\right)$ & [22] \\
\hline & & SRB/BEL-7402 & $10.9 \mu \mathrm{M}\left(\mathrm{IC}_{50}\right)$ & Adriamycin $0.4 \mu \mathrm{M}\left(\mathrm{IC}_{50}\right)$ & [22] \\
\hline & & SRB/HCT-116 & $11.3 \mu \mathrm{M}\left(\mathrm{IC}_{50}\right)$ & Adriamycin $0.2 \mu \mathrm{M}\left(\mathrm{IC}_{50}\right)$ & [22] \\
\hline & & $\mathrm{SRB} / \mathrm{HeLa}$ & $22.1 \mu \mathrm{M}\left(\mathrm{IC}_{50}\right)$ & Adriamycin $0.6 \mu \mathrm{M}\left(\mathrm{IC}_{50}\right)$ & [22] \\
\hline & & SRB/L-02 & $18.2 \mu \mathrm{M}\left(\mathrm{IC}_{50}\right)$ & Adriamycin $0.4 \mu \mathrm{M}\left(\mathrm{IC}_{50}\right)$ & [22] \\
\hline & & SRB/MGC-803 & $6.6 \mu \mathrm{M}\left(\mathrm{IC}_{50}\right)$ & Adriamycin $0.2 \mu \mathrm{M}\left(\mathrm{IC}_{50}\right)$ & {$[22]$} \\
\hline & & SRB/HO8910 & $9.7 \mu \mathrm{M}\left(\mathrm{IC}_{50}\right)$ & Adriamycin $0.4 \mu \mathrm{M}\left(\mathrm{IC}_{50}\right)$ & {$[22]$} \\
\hline
\end{tabular}


Table 2. Cont.

\begin{tabular}{|c|c|c|c|c|c|}
\hline Compound Name & Biological Activity & Assay, Organism, or Cell Line & Biological Results & Positive Control & Refs. \\
\hline & & SRB/NCI-H1975 & $11.2 \mu \mathrm{M}\left(\mathrm{IC}_{50}\right)$ & Adriamycin $0.3 \mu \mathrm{M}\left(\mathrm{IC}_{50}\right)$ & [22] \\
\hline & & SRB/U87 & $18.3 \mu \mathrm{M}\left(\mathrm{IC}_{50}\right)$ & Adriamycin $0.1 \mu \mathrm{M}\left(\mathrm{IC}_{50}\right)$ & [22] \\
\hline & & SRB/MDA-MB-231 & $13.2 \mu \mathrm{M}\left(\mathrm{IC}_{50}\right)$ & Adriamycin $0.2 \mu \mathrm{M}\left(\mathrm{IC}_{50}\right)$ & [22] \\
\hline \multirow[t]{4}{*}{ Chaetoviridin E (44) } & Cytotoxicity & SRB assay/BC1 & $5.6 \mu \mathrm{g} / \mathrm{mL}\left(\mathrm{IC}_{50}\right)$ & Ellipticine $0.26 \mu \mathrm{g} / \mathrm{mL}\left(\mathrm{IC}_{50}\right)$ & [60] \\
\hline & Cytotoxicity & SRB assay/NCl-H187 & $3.5 \mu \mathrm{g} / \mathrm{mL}\left(\mathrm{IC}_{50}\right)$ & Ellipticine $0.32 \mu \mathrm{g} / \mathrm{mL}\left(\mathrm{IC}_{50}\right)$ & {$[60]$} \\
\hline & Cytotoxicity & SRB assay/HepG-2 & $40.6 \mu \mathrm{M}\left(\mathrm{IC}_{50}\right)$ & Camptothecin $32.3 \mu \mathrm{M}\left(\mathrm{IC}_{50}\right)$ & [72] \\
\hline & Antimalarial & $\begin{array}{l}\text { Microculture radioisotope assay/ } \\
\text { P. falciparum (K1, MDR) }\end{array}$ & $2.9 \mu \mathrm{g} / \mathrm{mL}\left(\mathrm{IC}_{50}\right)$ & - & {$[60]$} \\
\hline 7-Epi-chaetoviridin E (45) & Caspase-3 inhibitory & Caspase- 3 enzymatic assay & $7.9 \mu \mathrm{M}\left(\mathrm{IC}_{50}\right)$ & Ac-DEVD-CHO 13.7 nM $\left(\mathrm{IC}_{50}\right)$ & [31] \\
\hline \multirow[t]{4}{*}{ Chaetoviridin F (47) } & Cytotoxicity & SRB assay/NCl-H187 & $4.5 \mu \mathrm{g} / \mathrm{mL}\left(\mathrm{IC}_{50}\right)$ & Ellipticine $0.32 \mu \mathrm{g} / \mathrm{mL}\left(\mathrm{IC}_{50}\right)$ & {$[60]$} \\
\hline & Cytotoxicity & MTT/P388 & $46.0 \mu \mathrm{M}\left(\mathrm{IC}_{50}\right)$ & 5-FU $1.9 \mu \mathrm{M}\left(\mathrm{IC}_{50}\right)$ & [43] \\
\hline & & MTT/HL-60 & $39.1 \mu \mathrm{M}\left(\mathrm{IC}_{50}\right)$ & 5-FU $2.3 \mu \mathrm{M}\left(\mathrm{IC}_{50}\right)$ & [43] \\
\hline & & MTT/L1210 & $43.7 \mu \mathrm{M}\left(\mathrm{IC}_{50}\right)$ & 5-FU $2.2 \mu \mathrm{M}\left(\mathrm{IC}_{50}\right)$ & [43] \\
\hline
\end{tabular}


The plates were developed by using toluene/EtOAc/formic acid (7:3:1), $\mathrm{CH}_{2} \mathrm{Cl}_{2} /$ $\mathrm{MeOH}$ (20:1), benzene/ethyl acetate (8:2), EtOAc $/ \mathrm{CH}_{2} \mathrm{Cl}_{2}$ (5:95), n-hexane/ethyl acetate (4:1), or EtOAc $/ \mathrm{CH}_{2} \mathrm{Cl}_{2}(2: 8)[29,42,60,63]$. The isolated metabolites can be purified by recrystallization from $\mathrm{MeOH}$ or $\mathrm{CHCl}_{3}: \mathrm{MeOH}$ until they show constant melting points.

The structures of isolated metabolites were determined through extensive spectroscopic analyses, including UV, IR, MS, and $1 \mathrm{D}\left({ }^{1} \mathrm{H},{ }^{13} \mathrm{C} \mathrm{NMR}\right.$, and DEPT) and 2D NMR (COSY, NOESY, ROESY, HMQC, HSQC, or HMBC).

The absolute configurations of these metabolites have been established with the aid of optical rotation sign, X-ray crystallography, CD (circular dichroism), the modified Mosher's method, and chemical transformation studies, including derivatization and degradation $[22,27,35,41,48,60,75]$. It has been reported that the absolute configuration at $\mathrm{C}$ (7) controls signs of the specific rotation [35]. Compounds with (S) C-11 and C-7 had negative optical rotation values; however, when C-7 was (R), the sign switched to positive with the same magnitude [50]. The absolute (S) configuration at C-7 was determined by the negative Cotton effect in the CD spectrum [58]. Mass spectra of these compounds displayed an isotopic peak $[\mathrm{M}+\mathrm{H}]^{+} /[\mathrm{M}+\mathrm{H}+2]^{+}$in a ratio 3:1, characterizing the presence of a single chlorine atom. Moreover, their IR spectrum exhibited characteristic bands for a hydroxyl group (3405-3450 $\left.\mathrm{cm}^{-1}\right)$, lactone $\left(1718-1780 \mathrm{~cm}^{-1}\right)$, and $\alpha, \beta$-unsaturated ketone $\left(1616-1684 \mathrm{~cm}^{-1}\right)$. Characteristic UV bands of a highly extended conjugation system were observed at 283-429 $\mathrm{nm}$.

\section{Biological Activities}

\subsection{Cytotoxic Activity}

Yamada et al. reported the isolation of chaetomugilins A (2), B (6), C (7), D (8), E (11), F (13), G (14), and H (15) from the culture broth of C. globosum associated with marine fish Mugil cephalus and assessed for their cytotoxic effects on P388 and HL-60 cell lines in the MTT assay (Figures 2 and 3; Table 2). It is noteworthy that compounds 7 and 13 exhibited remarkable cytotoxicity towards P388 and HL-60 cell lines $\left(\mathrm{IC}_{50} 3.6\right.$ and $3.3 \mu \mathrm{M}$ and 2.7 and $1.3 \mu \mathrm{M}$, respectively), nearly equal to that of 5-fluorouracil ( $\mathrm{IC}_{50} 1.7$ and $2.7 \mu \mathrm{M}$, respectively). While other compounds had moderate to weak cytotoxicity ( $\mathrm{IC}_{50}$ ranging from 6.8 to $24.1 \mu \mathrm{M}$ ) [38,39]. Further, 2, 7, and 13 displayed selective cytotoxicity towards a panel of 39 disease-related human cell lines, including breast, CNS, colon, lung, melanoma, ovary, kidney, stomach, and prostate cancer cells with range and delta values of 2 (1.24 and 1.13, respectively), 7 (1.19 and 0.71, respectively), and 13 (1.21 and 1.97, respectively) [38,40]. It was suggested that the existence of C-12-hydroxyl and C-3-methoxyl groups had little effect on the activity [40]. Evaluation of the differential cytotoxicity patterns using COMPARE revealed that the modes of action for 2, 7, and 13 might be different from those of other anticancer drugs [40]. On the other hand, chaetomugilins A (2) biosynthesized by C. globosum Z1 isolated from Broussonetia papyrifera bark had no in vitro effectiveness towards SMMC-7721, MG-63 and A-549 cell lines ( $\left.\mathrm{IC}_{50}>50 \mu \mathrm{g} / \mathrm{mL}\right)$ in the MTT assay in comparison to doxorubicin [44]. 
<smiles>C[C@@H](/C=C/C1=CC2=C(Cl)C(=O)[C@](C)(O)[C@H](O)[C@H]2CO1)[C@@H](C)O</smiles>

Chaetomugilin 106B-6 XXVIII (1)

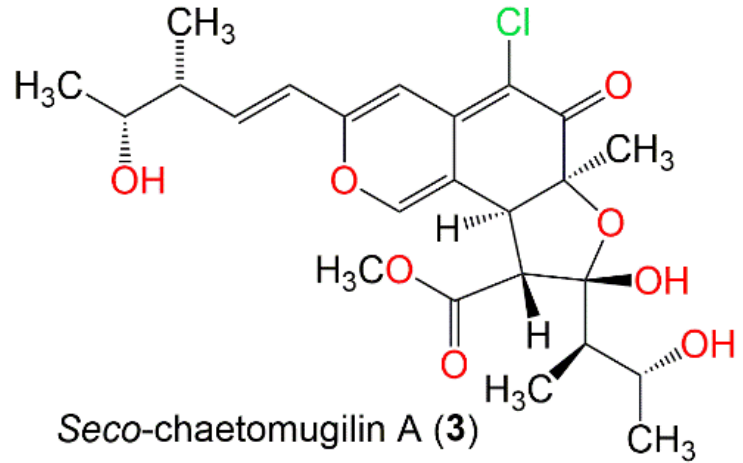

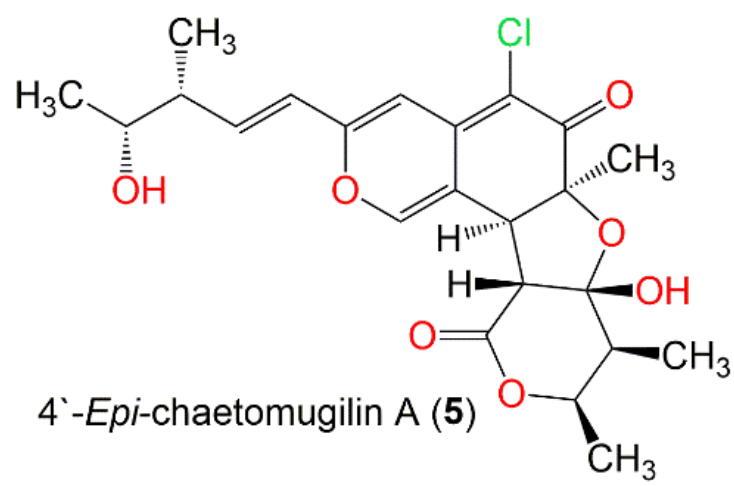<smiles>C[C@H](O)[C@@H](C)/C=C/C1=CC2=C(Cl)C(=O)[C@]3(C)OC4=C(C(=O)O[C@H](C)[C@H]4C)[C@H]3[C@@H]2CO1</smiles>

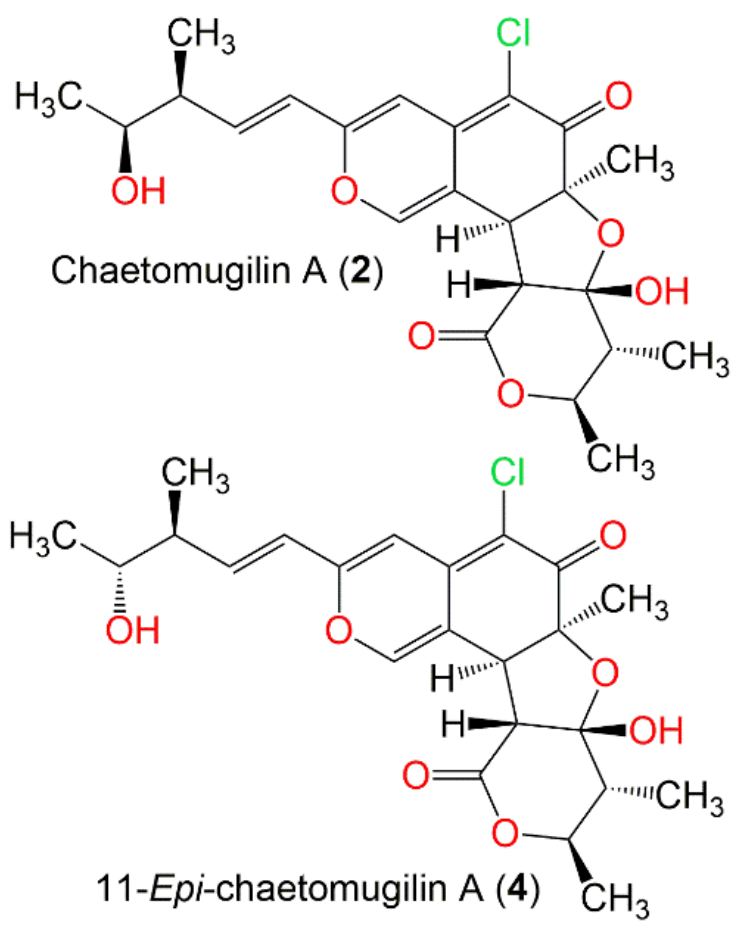

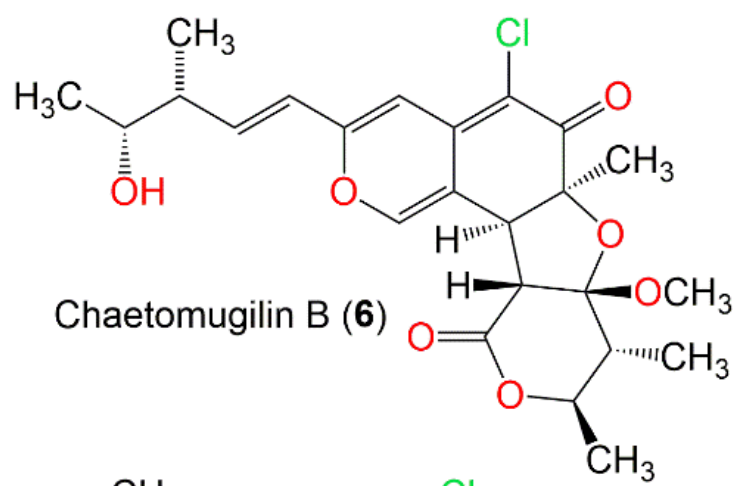<smiles></smiles>

Figure 2. Structures of compounds 1-8. 
<smiles>CC[C@H](C)/C=C/C1=CC2=C(Cl)C(=O)[C@]3(C)O[C@](O)([C@H](C)[C@H](C)O)[C@@H](C(=O)OC)[C@H]3C2=CO1</smiles>

Seco-chaetomugilin D (9) $\quad \mathrm{CH}_{3}$

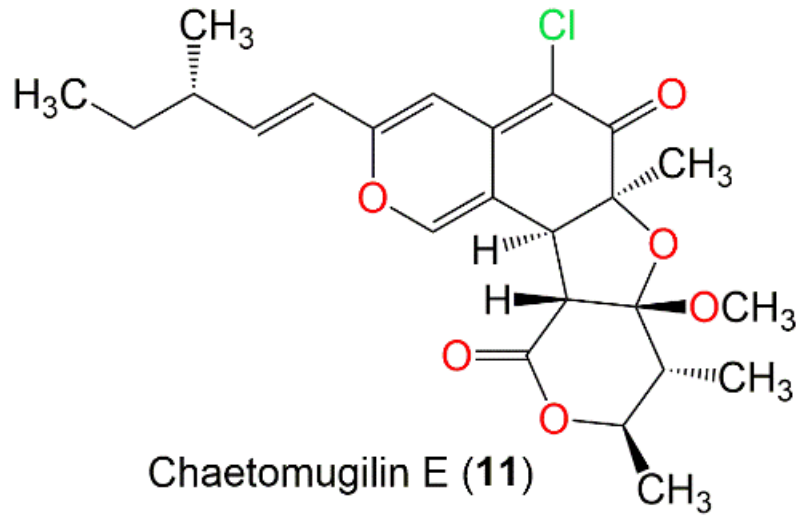<smiles>CC[C@H](C)/C=C/C1=CC2=C(Cl)C(=O)[C@]3(C)OC4=C(C(=O)O[C@H](C)[C@H]4C)[C@H]3C2=CO1</smiles><smiles></smiles>

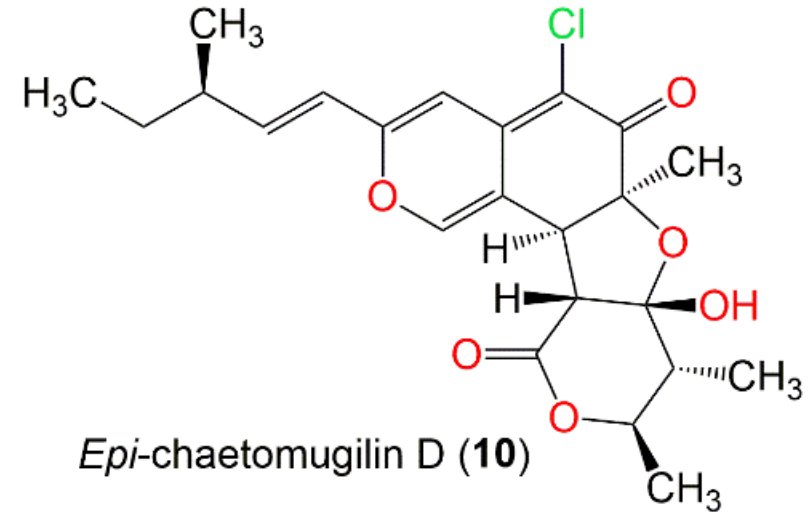<smiles>C/C=C(\C)C(=O)CC1C2=COC(/C=C/C(C)C(C)O)=CC2=C(Cl)C(=O)C1(C)O</smiles><smiles>COC(=O)C1=C([C@@H](C)[C@H](C)O)O[C@]2(C)C(=O)C(Cl)=C3C=C(/C=C/[C@@H](C)[C@H](C)O)OC=C3[C@H]12</smiles><smiles>C/C=C(\C)C(=O)C[C@H]1C2=COC(/C=C/[C@@H](C)[C@H](C)O)=CC2=C(Cl)C(=O)[C@]1(C)O</smiles>

Figure 3. Structures of compounds 9-16. 
C. globosum OUPS-T106B-6 isolated from M. cephalus yielded two new metabolites that demonstrated moderate cytotoxicity towards HL-60 and P388 cell lines $\left(\mathrm{IC}_{50}\right.$ ranged from 10.3 to $24.1 \mu \mathrm{M}$, respectively), compared to 5 -FU $\left(\mathrm{IC}_{50} 2.7\right.$ and $\left.1.7 \mu \mathrm{M}\right)$ in the MTT assay [41]: chaetomugilins $\mathrm{G}$ (14) and $\mathrm{H}$ (15).

In another study by Yamada et al. on the same fungus, two new compounds named seco-chaetomugilins A (3) and D (8) were separated. Compound 8 exhibited weak activity (IC50 38.6-53.6 $\mu \mathrm{M}$ ) towards HL-60, P388, KB, and L1210, compared with 5-FU $\left(\mathrm{IC}_{50} 1.7-6.0 \mu \mathrm{M}\right)$; however, 8 was inactive. It was suggested that the C-12-hydroxyl group decreased the activity [48].

Chaetomugilins I (16), J (18), 11-epichaetomugilin A (4), 4'-epichaetomugilin A (5), and 106B-6 XXVIII (1) were separated from C. globosum 106B-6 and assessed for cytotoxic activity towards P388, HL-60, L1210, and KB cell lines. Interestingly, compounds $\mathbf{1 6}$ and $\mathbf{1 8}$ had significant cytotoxicity ( $\mathrm{IC}_{50} 1.1-2.3 \mu \mathrm{M}$ for 16 and $2.8-12.8 \mu \mathrm{M}$ for 18) towards all cell lines equal to that of 5-FU ( $\left.\mathrm{IC}_{50} 1.1-7.7 \mu \mathrm{M}\right)$. In addition, 16 showed potent and selective cytotoxic activity towards a panel of 39 human cell lines. The other compounds exhibited moderate to marginal activity toward the tested cell lines [37].

Muroga et al. assessed the cytotoxicity of the new metabolites, chaetomugilins I-O (16 and 18-23), towards P388, HL-60, L1210, and KB cell lines using the MTT assay. Compounds $16,18-20,22$, and 23 revealed remarkable cytotoxicity ( $\mathrm{IC}_{50}$ ranged from 1.1 to $20.1 \mu \mathrm{M}$ ) towards all cell lines, compared to 5-FU ( $\left.\mathrm{IC}_{50} 1.1-7.7 \mu \mathrm{M}\right)$, whereas chaetomugilin $\mathrm{M}$ (21) was inactive. Particularly, compound $\mathbf{1 6}$ was more potent than 5-FU. Further, 16 had selective and potent cytotoxicity towards a panel of 39 human cell lines [58].

Furthermore, the new metabolites, 11- and 4'-epichaetomugilin A (4 and 5) purified from C. globosum isolated from M. cephalus, displayed moderate to weak cytotoxicity toward KB, P388, HL-60, and L1210 cell lines [35].

The new metabolites, chaetomugilins P-R (24-27) and 11-epi-chaetomugilin I (17), along with the formerly separated chaetomugilin I (16), were purified by the marine fish-associated C. globosum (Figures 4 and 5) [59]. Compounds 24, 17, and 16 possessed stronger cytotoxicity towards HL-60, P388, KB, and L1210 ( $\mathrm{IC}_{50} 1.1,1.1,2.3$, and $1.9 \mu \mathrm{M}$, respectively, for $16 ; 1.0,0.7,1.2$, and $1.6 \mu \mathrm{M}$, respectively, for 17 ; and $1.2,0.7,1.8$, and $1.5 \mu \mathrm{M}$, respectively, for 24$)$ cell lines than $5-\mathrm{FU}$ ( $\mathrm{IC}_{50} 2.7,1.7,7.7$, and $1.1 \mu \mathrm{M}$, respectively). The results indicated that the $\mathrm{C}-2^{\prime}-\mathrm{C}-4^{\prime}$ enone moiety is essential for activity. On the other hand, compounds 25-27 were weakly to moderately active towards all tested cancer cell lines $\left(\mathrm{IC}_{50} 32.0-80.2 \mu \mathrm{M}\right)$ [59].

The new metabolites, chaetomugilin S (28), T (29), and U (30), separated from C. globosum derived from M. cephalus, revealed moderate to high growth inhibition towards HL-60, P388, KB, and L1210 cell lines ( $\mathrm{IC}_{50}$ ranging from 34.4 to $94.9 \mu \mathrm{M}$ ), relative to 5-FU ( $\left.\mathrm{IC}_{50} 1.9-20.6 \mu \mathrm{M}\right)$ [43].

In 2019, Sun et al. evaluated the cytotoxicity of the new glutamine-containing derivatives, $\mathrm{N}$-glutarylchaetoviridins A-C $(35,39$, and 42$)$, in addition to chaetomugilins A (2) and $C$ (7) from the extract of deep-sea sediment-associated C. globosum HDN151398 toward BEL-7402, HeLa, HCT-116, L-02, HO8910, MGC-803, SH-SY5Y, U87, NCI-H1975, and MDAMB-231 cancer cells using the SRB method and towards HL-60 and K562 using MTT method (Figure 6). Chaetomugilins A (2) and C (7) and N-glutarylchaetoviridin C (42) exhibited powerful cytotoxicity towards all tested cell lines ( $\mathrm{IC}_{50} 5.7-27.1 \mu \mathrm{M}$ for 2, 6.6-26.6 $\mu \mathrm{M}$ for 7, and 6.6-26.5 $\mu \mathrm{M}$ for 42) compared to adriamycin ( $\left.\mathrm{IC}_{50} 0.1-0.6 \mu \mathrm{M}\right)$ [22]. Among them, N-glutarylchaetoviridin C (42) had a remarkable cytotoxicity toward MGC-803 and $\mathrm{HO}-8910$ ( $\mathrm{IC}_{50} 6.6$ and $9.7 \mu \mathrm{M}$, respectively) [22]. 
<smiles>C/C=C(\C)C(=O)C[C@H]1C2=COC(/C=C/[C@@H](C)[C@H](C)O)=CC2=C(Cl)C(=O)[C@]1(C)O</smiles><smiles>C/C=C(\C)C(=O)C[C@H]1C2=COC(/C=C/[C@H](C)CC)=CC2=C(Cl)C(=O)[C@]1(C)COCCO</smiles>

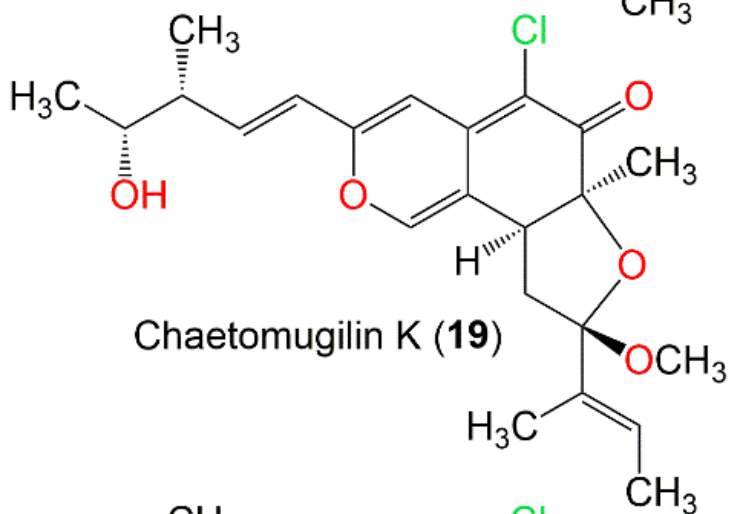

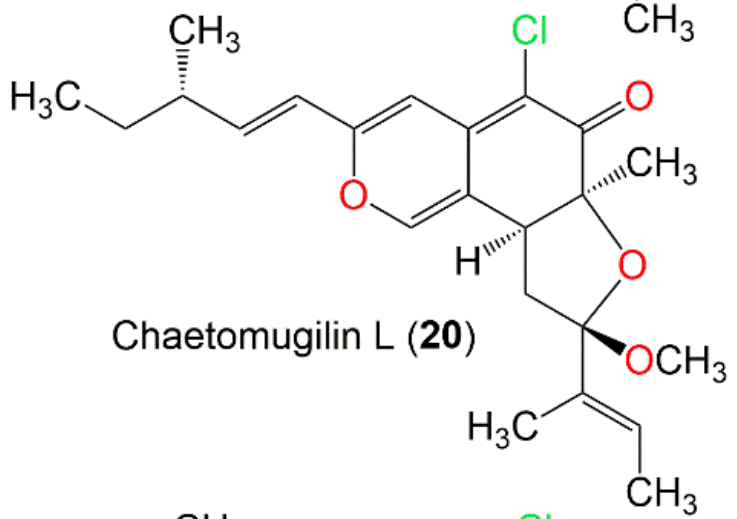<smiles>C=C[C@H](C)[C@H](C)O</smiles><smiles>C[C@@H](O)[C@@H](C)C(=O)[C@H]1C(=O)O[C@]2(C)C(=O)C(Cl)=C3C=COC=C3[C@H]12</smiles><smiles>C/C=C(\C)C(=O)[C@H]1C(=O)O[C@]2(C)C(=O)C(Cl)=C3C=C(/C=C/[C@@H](C)[C@@H](C)O)OC=C3[C@]12C</smiles><smiles>C/C=C(\C)C(=O)[C@]1(C)C(=O)O[C@]2(C)C(=O)C(Cl)=C3C=C(/C=C/[C@H](C)CC)OC=C3[C@@H]21</smiles>

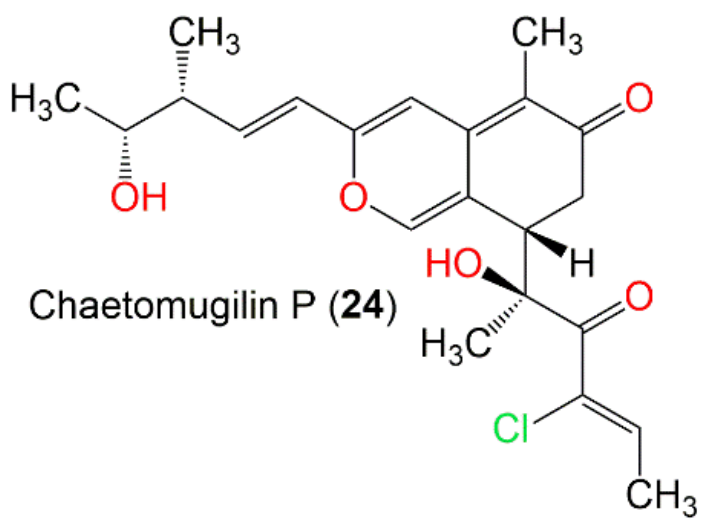

Figure 4. Structures of compounds 17-24. 
<smiles>CC(C(=O)C[C@@H]1[C@H]2COC(/C=C/[C@H](C)[C@H](C)O)=CC2=C(Cl)C(=O)[C@]1(C)O)[C@H](C)O</smiles><smiles>C[C@@H](/C=C/C1=CC2=C(Cl)C(=O)[C@](C)(O)[C@H](O)[C@H]2CO1)[C@@H](C)O</smiles>

Chaetomugilin R (26)

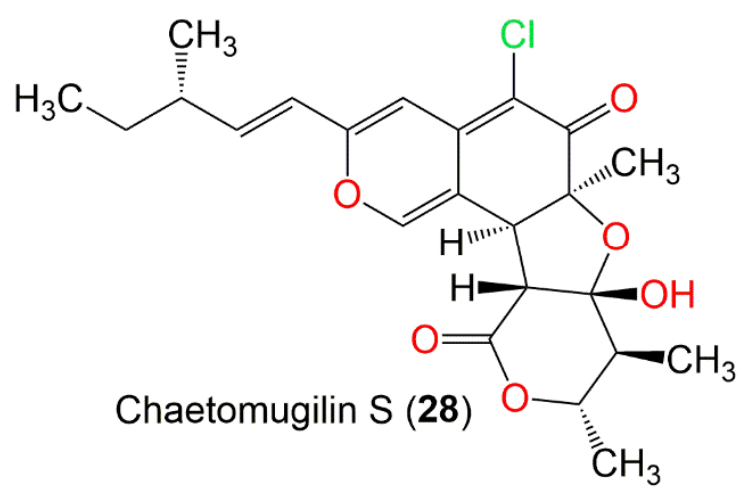<smiles>CC[C@H](C)/C=C/C1=CC2=C(Cl)C(=O)[C@@]3(C)OC(=O)C(C(=O)[C@H](C)[C@@H](C)O)=C3C2=CO1</smiles>

Chaetomugilin T (29)

Chaetomugilin S (27)<smiles>C[C@H](O)[C@@H](C)/C=C/C1=CC2=CC(=O)[C@]3(C)OC[C@H](C=C23)O1</smiles><smiles>CC1OC(=O)[C@@H](C)[C@@](C)(O)[C@@H]1C</smiles>

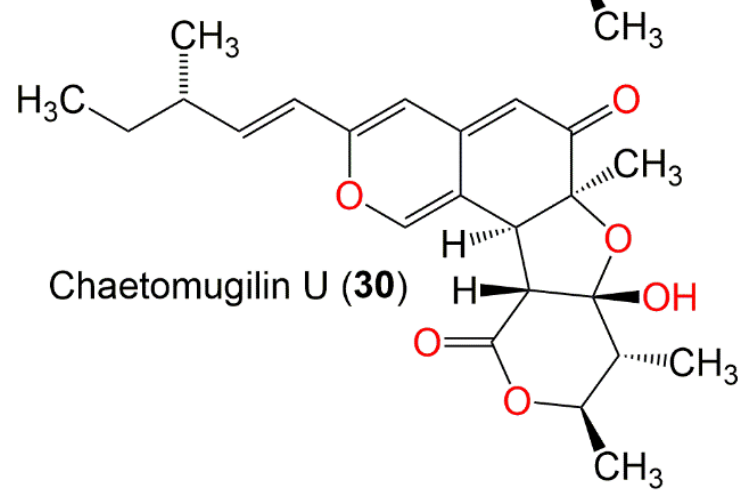

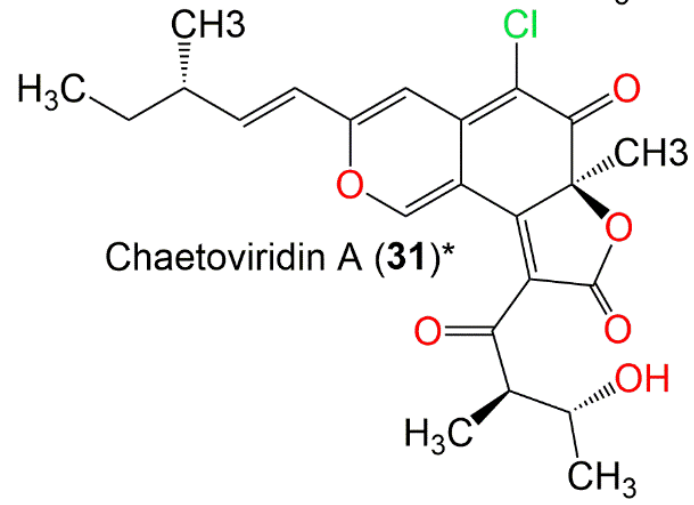

Figure 5. Structures of compounds 25-31 (* Revised by Makrerougras et al). 
<smiles>CC[C@H](C)/C=C/C1=CC2=C(Cl)C(=O)[C@]3(C)OC(=O)C(C(=O)[C@H](C)[C@H](C)O)=C3C2=CO1</smiles><smiles>CC[C@H](C)/C=C/C1=CC2=C(Cl)C(=O)[C@@]3(C)OC(=O)C(C(=O)[C@H](C)[C@@H](C)O)=C3C2=CO1</smiles><smiles>CC[C@H](C)/C=C/C1=CC2=C(Cl)C(=O)[C@@]3(C)OC(=O)C(C(=O)[C@H](C)[C@@H](C)O)=C3C2=CO1</smiles><smiles>CC[C@H](C)/C=C/C1=CC2=C(Cl)C(=O)[C@@]3(C)OC(=O)C(C(=O)[C@H](C)[C@H](C)O)=C3C2=CO1</smiles>

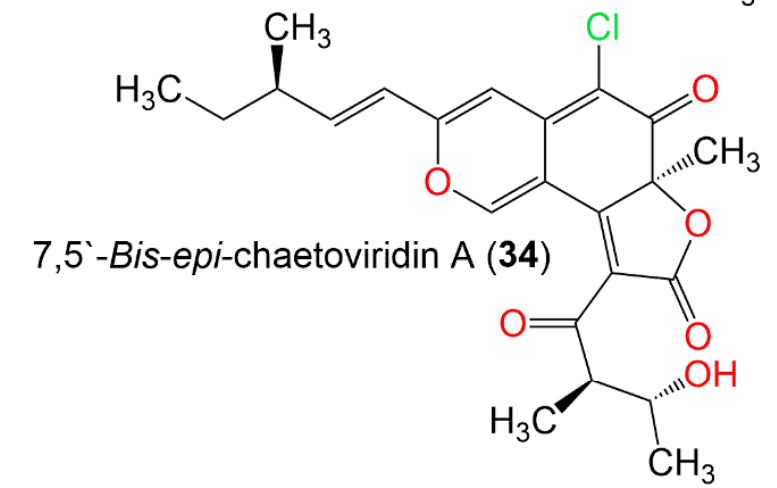

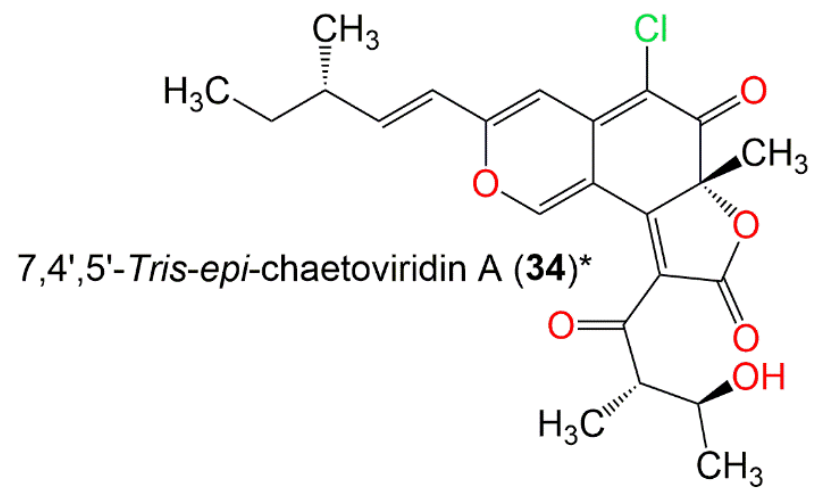<smiles>CC[C@H](C)/C=C\C1=CC2=C(Cl)C(=O)[C@@]3(C)OC(=O)C(C(=O)[C@H](C)[C@H](C)OC)=C3C2=CN1[C@@H](CCC(=O)OC)C(=O)OC</smiles><smiles>CC[C@H](C)/C=C/C1=CC2=C(Cl)C(=O)[C@@]3(C)OC(=O)C(C(=O)[C@H](C)[C@H](C)O)=C3C2=CN1CCO</smiles>

4`-Epi-N-2-Hydroxyethyl-azachaetoviridin A (36)

Figure 6. Structures of compounds 32-36 ( ${ }^{*}$ Revised and renamed by Makrerougras et al.). 
Chaetomugilin A (2), 11-epi-chaetomugilin A (4), and chaetomugilin D (8) displayed no noticeable cytotoxic activity $\left(\mathrm{IC}_{50}>40 \mu \mathrm{M}\right)$ toward HepG-2, A549, and HeLa in the MTT assay compared to etoposide ( $\mathrm{IC}_{50} 16.11,16.46$, and $15.00 \mu \mathrm{M}$, respectively) [46].

Wani et al. reported that seco-chaetomugilin D (9) isolated from C. cupreum had cytotoxicity towards MCF-7 (inhibition ranging from $25.25 \%$ to $75.25 \%$ after $24 \mathrm{~h}$ and from $41.5 \%$ to $99 \%$ after $38 \mathrm{~h}$ at $3.12-50 \mu \mathrm{g} / \mathrm{mL}$ ). Further, it increased mitochondrial membrane depolarization ( $16.45 \%$ and $32.25 \%$ at 5 and $15 \mu \mathrm{g} / \mathrm{mL}$, respectively) and induced ROS production ( $19.6 \%$ and $26.2 \%$ at 5 and $15 \mu \mathrm{g} / \mathrm{mL}$, respectively) in comparison to untreated cells. Therefore, it caused cell death via induction of mitochondrial ROS production and membrane depolarization [29].

C. globosum isolated from Ginkgo biloba leaves yielded chaetomugilin D (8) and chaetomugilin A (2), which demonstrated significant toxicity toward brine shrimp (Artemia salina) larvae after $24 \mathrm{~h}$ (mortality rates 75.2 and $78.3 \%$, respectively, at $10 \mu \mathrm{g} / \mathrm{mL}$ ) [42].

Hu et al. proved that chaetomugilin J (18) combined with low-dose cisplatin decreased cell viability and boosted cisplatin-produced apoptosis in ovarian A2780 cells independently of the endoplasmic reticulum apoptotic pathway. It significantly induced mitochondrial dysfunction and apoptosis via increasing the intracellular and mitochondrial ROS levels and decreasing mitochondrial membrane potential. It also prohibited parkin/PINK1 induced mitophagy, resulting in weakening the mitophagy protective effect that led to apoptosis and increased sensitivity to cisplatin [34]. Chaetomugilin D (8), chaetoviridin A (31), and chaetoviridin E (44) purified from a deep sea-derived Chaetomium sp. NA-S01-R1 displayed moderate to weak cytotoxicity toward HeLa, A549, and HepG2, in comparison to doxorubicin ( $\mathrm{IC}_{50} 0.1-1.1 \mu \mathrm{M}$ ) using the CCK-8 assay [25] (Figure 7). Additionally, chaetoviridin A (31) and chaetoviridin E (44) were inactive towards AGS and MGC803 [71].

In addition, chaetoviridins $\mathrm{E}$ and $\mathrm{F}$ (44 and 47 ) had cytotoxicity toward NCI-H187, KB, and $\mathrm{BC} 1$ ( $\mathrm{IC}_{50}$ ranging from 3.5 to $13.4 \mu \mathrm{g} / \mathrm{mL}$ ), in comparison to ellipticine ( $\mathrm{IC}_{50}$ ranging from 0.26 to $0.36 \mu \mathrm{g} / \mathrm{mL})[60]$.

Chaetoviridin A (31) $\left(2 \mu \mathrm{M}, \mathrm{ID}_{50} 0.6 \mu \mathrm{M}\right)$ inhibited the inflammatory activity of TPA (12-O-tetradecanoylphorbol-13-acetate, $1 \mu \mathrm{g}$ ) in mice ( $\left.\operatorname{ID}_{50} 0.6 \mu \mathrm{M}\right)$. Furthermore, it markedly suppressed the promoting effect of TPA on skin tumor formation in mice initiated with 7,12-dimethylbenz[a]an-thracene $(50 \mu \mathrm{g})$. It was proposed that it inhibited TPAtumor promotion in a two-stage carcinogenesis model in mice due to its anti-inflammatory potential [76].

C. globosum TY1 associated with Ginkgo biloba barks yielded chaetoviridins B (38) and $\mathrm{E}$ (44) and 5'-epi-chaetoviridin A (33). Compounds 44 and 33 had moderate cytotoxicity towards HepG-2 ( $\mathrm{IC}_{50} 40.6$ and $35.3 \mu \mathrm{M}$, respectively) compared to camptothecin $\left(\mathrm{IC}_{50} 32.3 \mu \mathrm{M}\right)$ in the SRB assay, and $\mathbf{3 8}$ was inactive [72].

C. globosum isolated from Artemisia desterorum roots yielded chaetoviridin E (44) and chaetoviridin A (31). They had no cytotoxic activity toward A549, HCT116, and HepG2 cancer cells [68]. The new metabolite, epi-chetomugilin D (10), along with chaetovirdins A (31), B (37), and E (44) and chetomugilins D (8) and J (18) were purified from C. globosum associated with Adiantum capillus-veneris. Chaetovirdin E (5 $\mathrm{\mu g} / \mathrm{mL})$ exhibited cytotoxicity towards $\mathrm{CaCO}_{2}$ and HepG2 cancers cells with $30 \%$ and $59 \%$ inhibition, respectively [49]. 
<smiles>CC[C@H](C)/C=C/C1=CC2=C(Cl)C(=O)[C@@]3(C)OC(O)(C(C)C(C)O)C(C(=O)O)C3C2=CO1</smiles><smiles>CC[C@H](C)/C=C/C1=CC2=C(Cl)C(=O)[C@]3(C)O[C@]4(O)[C@@H](C)[C@@H](C)OC(=O)[C@@H]4[C@H]3[C@H]2CO1</smiles>

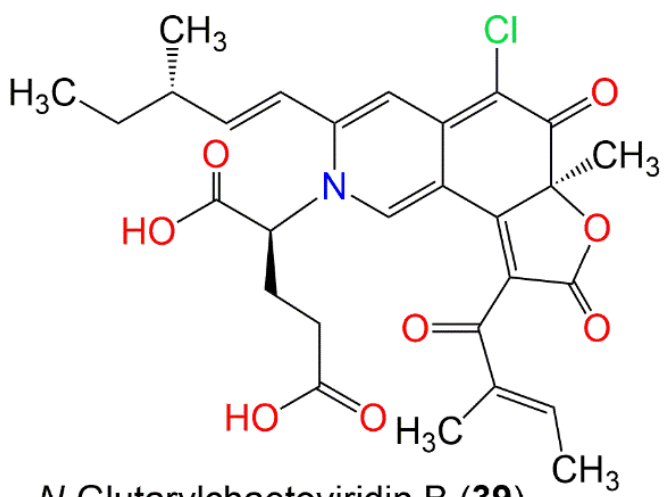<smiles>C/C=C(\C)C(=O)C1=C2C3=CN([C@@H](CCC(=O)OC)C(=O)OC)C(/C=C/[C@@H](C)CC)=CC3=C(Cl)C(=O)[C@@]2(C)OC1=O</smiles><smiles>C[C@H](O)[C@@H](C)/C=C/C1=CC2=C(Cl)C(=O)[C@]3(C)OC(=O)[C@H](C(=O)[C@H](C)[C@H](C)O)[C@H]3[C@H]2CO1</smiles>

N-Glutarylchaetoviridin C (42)

$12 \beta$-Hydroxychaetoviridin C (41)<smiles>CC(O)C(C)/C=C/C1=CC2=C(Cl)C(=O)C3(C)OC(O)(C(C)C(C)O)C(C(=O)O)C3C2=CO1</smiles><smiles>C/C=C(\C)C(=O)C1=C2C3=COC(/C=C/[C@H](C)CC)=CC3=C(Cl)C(=O)[C@@]2(C)OC1=O</smiles>

Figure 7. Structures of compounds $37-44$ ( ${ }^{*}$ Revised Kingsland and Barrow). 


\subsection{Antimicrobial Activity}

The liquid culture of C. globosum DAOM-240359 isolated from an indoor air sample collected from Ottawa, Ontario, Canada produced new nitrogen-containing chaetoviridins, 4'-epi-N-2-hydroxyethyl-azachaetoviridin A (36) and N-2-butyric-azochaetoviridin E (46), along with chaetoviridin A (31) and chaetomugilin D (8). Compound 36 is a nitrogenous derivative of $\mathbf{3 1}$ with an N-2-hydroxy ethyl chain and (R) configuration at C-4' instead of the $(S)$ configuration of 31 . Compound 46 is a nitrogenous derivative of chaetoviridin $\mathrm{E}$ (44) with a C-2 $\gamma$-aminobutyric acid moiety. This represents the first reported azaphilone with a 3-methyl-1-pentyl group and an N-2 side chain. Compounds 8 and 46 significantly reduced the growth of Pseudomonas putida and B. subtilus (conc. $20 \mu \mathrm{M}$ ) in the quantitative growth inhibition assay. Additionally, they showed the same effectiveness as chloramphenicol at $200 \mu \mathrm{M}$. On the other hand, 8 and 46 showed antifungal activity towards Saccharomyces cerevisiae at $2 \mathrm{mM}$ and $200 \mu \mathrm{M}$, respectively. However, 31 exerted its antibacterial activity at $200 \mu \mathrm{M}$ [50]. Chaetovirdin A (31) possessed weak inhibitory potential towards induction of chlamydospore-like cells of the plant pathogen Cochliobolus lunatus (40-50\% at $100 \mu \mathrm{g} /$ disc), and prohibition of the rice-blast fungus Pyricuralia oryzae's growth $\left(\mathrm{IC}_{50} 2.5 \mu \mathrm{g} / \mathrm{mL}\right)$ [27]. The new metabolites, 5'-epichaetoviridin A (33), 4 '-epichaetoviridin F (48), 12 $\beta$-hydroxychaetoviridin C (41), and chaetoviridins G-I (49, 51, and 52), along with chaetoviridins A-E (31,37, 40,43, and 44) and $4^{\prime}$-epichaetoviridin A (32) separated from the endophytic fungus C. globosum, were assessed in an in vivo pathogenicity assay that involved the infection of Caenorhabditis elegans with Enterococcus faecalis (Figure 8). None of them possessed a significant ability to promote nematode survival [36]. Park et al. stated that chaetoviridins A (31) and B (37) also possessed growth inhibitory activity against Magnaporthe grisea (rice blast) and Pythium ultimum (wheat leaf rust) mycelia (MICs 1.23 and 33.3 and 1.23 and $33.3 \mu \mathrm{g} / \mathrm{mL}$, respectively) in vitro [61]. They also exhibited strong in vivo antifungal activity against $M$. grisea and Puccnicia recondite (wheat leaf rust) [36]. Chaetoviridin A (Conc. $62.5 \mu \mathrm{g} / \mathrm{mL}$ ) inhibited rice blast development by $>80 \%$, but it had moderate control (50\%) of tomato late blight at $125 \mu \mathrm{g} / \mathrm{mL}$. Therefore, they can control wheat leaf rust rice blast and tomato late blight $[36,61]$. Chaetoviridin A (31), chaetoviridin E (44), and chaetomugilin D (8) separated from a deep sea-derived Chaetomium sp. NA-S01-R1 had weak to moderate antibacterial effectiveness against Vibrio strains (V. vulnificus, $V$. rotiferianus, and $V$. campbellii) (MIC 15.4-32.3 $\mu \mathrm{g} / \mathrm{mL}$ ) and MRSA (MICs 15.2-32.4 $\mu \mathrm{g} / \mathrm{mL}$ ), compared to erythromycin (MIC 2.0-7.7 $\mu \mathrm{g} / \mathrm{mL}$ ) and chloramphenicol (MIC 7.5-7.6 $\mu \mathrm{g} / \mathrm{mL}$ ) [25].

Chaetoviridins B (38) and E (44) displayed antibacterial activity towards E. faecalis and S. aureus [74]. Chaetoviridins A (31) and B (34) also had antimicrobial activity against B. subtilis, Rhizoctonia solani, and E. coli (IZDs 15 and $14 \mathrm{~mm}$, respectively, towards all strains) [63].

Yan et al. reported that chaetoviridin A (31) exhibited significant antifungal potential (EC $501.97 \mu \mathrm{g} / \mathrm{mL}$ ) towards Sclerotinia sclerotiorum, which causes rape Sclerotinia rot (RSR). Further, 31 displayed in vivo protective efficacy $(64.3 \%$, dose $200 \mu \mathrm{g} / \mathrm{mL})$ towards RSR, comparable to that of carbendazim (69.2\%). Additionally, it had antifungal activity towards Botrytis cinerea, Phytophthora capsici, Fusarium graminearum, and F. moniliforme (inhibition rates $69.1,60.7,77.0$, and 59.2\%, respectively) [73].

C. globosum CEF-082, isolated from cotton plants, produced chaetoviridin A (31), which possesses significant antifungal activity towards Verticillium dahlia, which causes cotton Verticillium wilt (CVW). It induced mycelial deformation and cell necrosis, increased NO and ROS production, prohibited the germination of microsclerotia of $V$. dahliae, and boosted the cotton defensive response [69].

Chaetoviridin A (31), 5'-epichaetoviridin A (33), and chaetoviridin E (44) were separated from the soil-associated C. globosum 22-10. They showed significant inhibitory effectiveness (inhibition 32.31\%, 15.38\%, and 13.85\%, respectively) at $100 \mu \mathrm{g} / \mathrm{mL}$ towards Bipolaris sorokiniana, a soil-borne pathogen that commonly causes wheat root rot. It is noteworthy that chaetoviridin A had the same inhibitory efficiency as the carbendazim 
(3 mg/mL), suggesting its potential to be a biocontrol agent for B. sorokiniana [67]. Further, chaetoviridin A identified from an EtOAc extract of $C$. globosum F211_UMNG isolated from P. heptaphyllum was active towards F. oxysporum [66]. Chaetomugilin D (8) and chaetoviridin A (31) $(200 \mu \mathrm{M})$ significantly reduced the growth of B. subtilis and Psuedomonas putida [55].

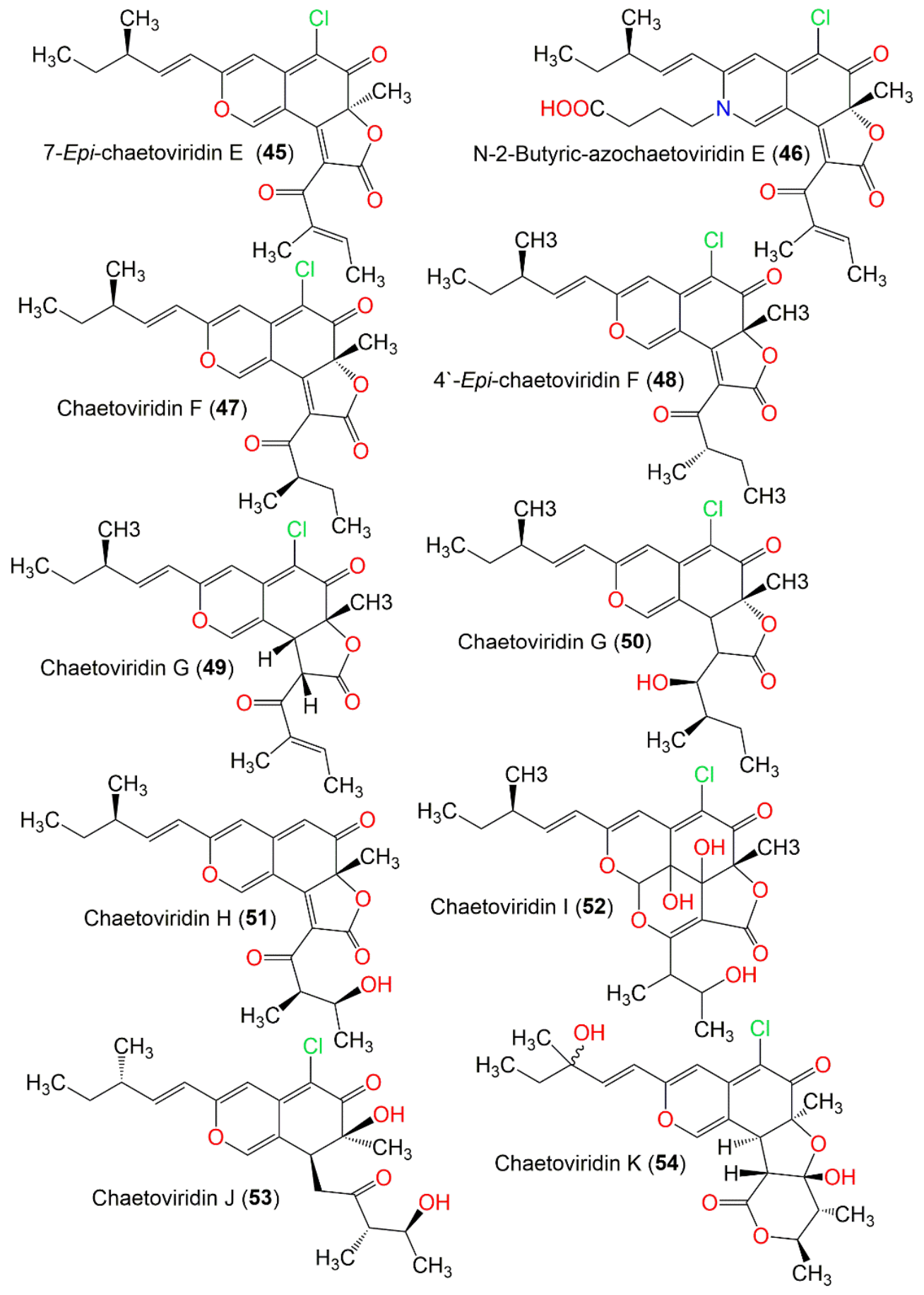

Figure 8. Structures of compounds 45-54.

\subsection{Phytotoxic Activity}

The EtOAc extract of $C$. globosum associated with Amaranthus viridis yielded chaetomugilin D (8) and chaetomugilin J (18), which exhibited phytotoxic potential against lettuce (Lactuca sativa) seed germination with $\mathrm{IC}_{50} 24.2$ and $22.6 \mathrm{ppm}$, respectively, for root growth inhibition, and $\mathrm{IC}_{50} 27.8$ and $21.9 \mathrm{ppm}$, respectively, for shoot growth inhibition. The results revealed the potential of these metabolites as herbicides or weedicides that can replace 
hazardous synthetic compounds [51]. Chaetomugilin A (2), D (8), S (28), I (16), J (18), Q (25), and $\mathrm{O}$ (23) isolated from C. globosum TY1 exhibited allelopathic activity towards Brassica campestris, Cucumis sativus, Eruca sativa, Daucus carota, Lactuca sativa, Scrophularia ningpoensis, Brassica rapa, and Spinacia oleracea. Among them, 23 exhibited higher germination and root and shoot elongation inhibitory potential with lower $\mathrm{IC}_{50}$ values and higher response indexes than glyphosate (positive control). Moreover, 2, 8, and 28 exhibited similar or better inhibitory effects than glyphosate. At the same time, 2, 8, and 23 were more powerful growth inhibitors than $\mathbf{1 6}, \mathbf{1 8}$, and 25 , which could be attributed to the existence of a tetrahydrofuran moiety. On the other hand, 23 had a higher growth-suppression effect than those of $\mathbf{2}, \mathbf{8}$, and $\mathbf{2 8}$, suggesting that the lactone rings may reduce the inhibitory effects [45].

\subsection{Antimalarial and Antimycobacterial Activities}

Phonkerd et al. isolated new derivatives, chaetoviridins E and F (44 and 47) and 5 -epi-chaetoviridin A (33), together with chaetoviridin A (31) from C cochliodes VTh01 and $C$. cochliodes $C$ Th05. Compound $\mathbf{4 4}$ showed antimalarial activity against $P$. falciparum $\left(\mathrm{IC}_{50} 2.9 \mu \mathrm{g} / \mathrm{mL}\right.$ ) - and was compared to artemisinin ( $\mathrm{IC}_{50} 0.001 \mu \mathrm{g} / \mathrm{mL}$ ) - using the microculture radioisotope technique [60]. Additionally, $\mathbf{4 4}$ and $\mathbf{4 7}$ displayed weak antimycobacterial potential towards M. tuberculosis (MIC 50 and $100 \mu \mathrm{g} / \mathrm{mL}$, respectively), in comparison to isoniazid and kanamycin sulfate in the microplate Alamar Blue assay (MABA) [60].

\subsection{Anti-Inflammatory Activity}

Chaetomugilin D (8) from C. globosum isolated from damp building materials notably increased TNF- $\alpha$ production in RAW 264.7 murine macrophages. Therefore, 8 contributes to the non-allergy-linked respiratory disorders such as non-allergic asthma and rhinitis for people working and living in damp buildings [52]. Two new derivatives, chaetoviridins J (53) and K (54), along with 11-epi-chaetomugilin I (17) and chaetomugilins E (11), F (13), I (16), J (18), and N (22) biosynthesized by C. globosum were isolated from Wikstroemia uva-ursi leaves. Their structures were verified by NMR, Mosher's method, X-ray diffraction, and CD. Chaetoviridin K (54) was separated as a mixture of diastereoisomers that could not be purified by chiral columns. Their potential to inhibit TNF- $\alpha$-induced NF- $k B$ and NO (nitric oxide) production in the LPS-stimulated RAW 264.7 cells was assessed. Compounds 16 and 17 remarkably suppressed TNF- $\alpha$-induced NF- $\mathrm{kB}$ activity ( $\mathrm{IC}_{50} 0.9 \mu \mathrm{M}$, at $\left.50 \mu \mathrm{M}\right)$, in comparison to BAY-11 $\left(\mathrm{IC}_{50} 2.0 \mu \mathrm{M}\right)$ and TPCK $\left(\mathrm{IC}_{50} 3.8 \mu \mathrm{M}\right)$, whereas 11, 13, and 18 possessed moderate inhibitory activity ( $\mathrm{IC}_{50}$ ranging from 5.1 to $11.6 \mu \mathrm{M}$, conc. $50 \mu \mathrm{M}$ ). In addition, 11, 13, 16-18, and 53 strongly prohibited NO production (74.2-99.9\%). It is noteworthy that $\mathbf{1 6}$ and $\mathbf{1 7}$ powerfully suppressed $\mathrm{NO}$ release $\left(\mathrm{IC}_{50} 0.3\right.$ and $0.8 \mu \mathrm{M}$, respectively) more than $\mathrm{N}$-monomethyl-L-arginine $\left(\mathrm{IC}_{50} 25.1 \mu \mathrm{M}\right)$. On the other hand, 11, 13, and 18 also considerably inhibited $\mathrm{NO}$ production $\left(\mathrm{IC}_{50}\right.$ values ranging from 1.9 to $5.8 \mu \mathrm{M})$; however, 54 exhibited a weak effect [56].

\subsection{Antidiabetic, Antioxidant, and Antiviral Activities}

Chaetomugilins A (2), I (16), J (18), and Q (25) and chaetoviridins B (38) and J (53) purified from the EtOAc extract of C. globosum TY-1 isolated from Ginkgo biloba bark were tested for $\alpha$-amylase and $\alpha$-glucosidase inhibitory activity. Chaetoviridin B (38) had promising $\alpha$-glucosidase inhibitory potential $\left(\mathrm{IC}_{50} 6.328 \mu \mathrm{M}\right)$, compared to acarbose $\left(\mathrm{IC}_{50} 54.7 \mu \mathrm{M}\right)$. The other compounds displayed no $\alpha$-amylase or $\alpha$-glucosidase inhibitory activity $\left(\mathrm{IC}_{50}>50 \mu \mathrm{M}\right)$ compared to acarbose ( $\mathrm{IC}_{50} 13.7$ and $54.7 \mu \mathrm{M}$, respectively) [47]. Chaetoviridin B (38) and chaetomugilin A (2) have the same skeleton except for the 2 having one more hydroxyl group in the side chain, revealing that the group is detrimental for $\alpha$-glucosidase inhibition [47]. Chaetoviridins A (31) and B (37) purified from an EtOAc extract of C. globosum exhibited noticeable antioxidant potential on TLC using DPPH [63]. Additionally, chetomugilin D (8) and its analog epi-chetomugilin D (10) possessed antiviral activity towards HSV-2 (inhibition $33.3 \%$ and $40.7 \%$, respectively, at $25 \mu \mathrm{g} / \mathrm{mL}$ [49]. 


\subsection{Caspase-3 and Monoamine Oxidase (MAO) Inhibitory Activities}

Caspase-3 (cysteine aspartyl-specific protease-3) is one of the executioners in caspaselinked apoptosis that is activated in nearly every apoptosis model [77]. It is a prominent therapeutic target for excessive apoptosis-associated disorders, such as ischemic damage and neurodegenerative disorders (e.g., Huntington's and Alzheimer's diseases) and autoimmune disorders [78]. New azaphilones, chaetomugilin S (27), 7,5'-bis-epi-chaetoviridin A (34), and 7-epi-chaetoviridin E (45), purified from the lichen-associated C. elatum 89-1-3-1, were isolated from Ramalina calicaris. Their absolute configurations were assigned by CD experiments and X-ray crystallography. They exhibited caspase- 3 inhibitory potential ( IC $_{50}$ $20.6,10.9$, and $7.9 \mu \mathrm{M}$, respectively) in the cysteine aspartyl-specific protease-3 enzymatic assay compared with Ac-DEVD-CHO ( $\left.\mathrm{IC}_{50} 13.7 \mathrm{nM}\right)$ [31]. On the other hand, 31 possessed weak MAO inhibitory potential (IC50 $\left.1.2 \times 10^{-2} \mathrm{~g} / \mathrm{mL}\right)$ [27].

\subsection{Cholesteryl Ester Transfer Protein Inhibitory Activity}

CETP allows the transfer and exchange of neutral lipids such as CE (cholesteryl ester) and TG (triacylglycerol) between plasma and lipoproteins. It is proven to play important role in atherosclerosis [79]. Tomoda et al. reported that chaetoviridin B (37) showed CETP (cholesteryl ester transfer protein) inhibitory activity with an $\mathrm{IC}_{50}<6.3 \mu \mathrm{M}$, whereas chaetoviridin A (31) had moderate inhibitory activity $\left(\mathrm{IC}_{50} 31.6 \mu \mathrm{M}\right)$ [80]. It was indicated that the existence of an electrophilic enone(s) and/or ketone(s) at both C-8 and C-6 of isochromane core is substantial for eliciting activity [80].

\subsection{Anti-SARS-CoV-2 Activity}

The COVID-19 pandemic has affected global health since 2019. COVID-19 can lead to acute respiratory distress syndrome [81]. It is produced by a novel type of coronavirus $(\mathrm{CoV})$ called SARS-CoV-2 that was first found in Wuhan City, China, and then spread worldwide $[82,83]$. It is considered a highly pathogenic $\mathrm{CoV}$ in the human population. The SARS-CoV-2 genome encodes two polyproteins which are processed by a 3C-like protease (3CLpro) and a papain-like protease [84]. 3CLpro (3C-like protease) and PLpro (papain-like protease) are needed for processing the polyproteins into mature nonstructural proteins, such as helicase and RdRp (RNA-dependent RNA polymerase), which are substantial for viral replication and transcription [85]. 3CLpro has high substrate specificity and is also referred to as Mpro (main protease) [86]. 3CLpro's substrate specificity makes this enzyme an ideal target for developing broad-spectrum antiviral agents [87]. Its inhibitors are expected to have selective toxicity towards the virus [88].

Fungi are a treasure that can provide a remarkable pool of secondary metabolites with antiviral activities [89]. The characterization and discovery of antiviral fungal metabolites is an emerging and promising research field. Recently, many reports have been published on the structure-based virtual screening approach for the repurposing of natural metabolites, hoping to accelerate and assist in the discovery of agents for COVID-19 treatment [82]. We carried out a computational study on the reported fungal chaetomugilins and chaetoviridins was carried out to identify their 3CLpro inhibitory potential, using docking calculations and MD simulations (Tables 3-7). Three crystal structures containing non-covalent inhibitors for the protease (PDB entry: 6W81, 6M2N, and 7K0F) were selected. All the listed metabolites were docked with extra precision for maximum accuracy. The docking method was validated by redocking the inhibitors that co-crystallized with 6W81, $6 \mathrm{M} 2 \mathrm{~N}$, and 7K0F; and RMSD values were within an acceptable range and less than $1.50 \AA$. All the redocked inhibitors revealed the same binding interaction with the active site in the original pose. Further, in silico ADMET (drug absorption, distribution, metabolism, excretion, and toxicity) predictions of the properties of the investigated compounds were carried out. Finally, a molecular dynamics simulation was conducted to evaluate the nature of the ligand-target interaction under simulated physiological conditions for the most compatible drug-like molecule that could be used in pursuit of a truly adequate medication for COVID-19. 
Table 3. In silico screening results of top candidates of the reported chaetomugilins and chaetoviridins against SARS-CoV-2 3CL protease (PDB: 6W81, 6M2N, and $7 \mathrm{~K} 0 \mathrm{~F}$ ).

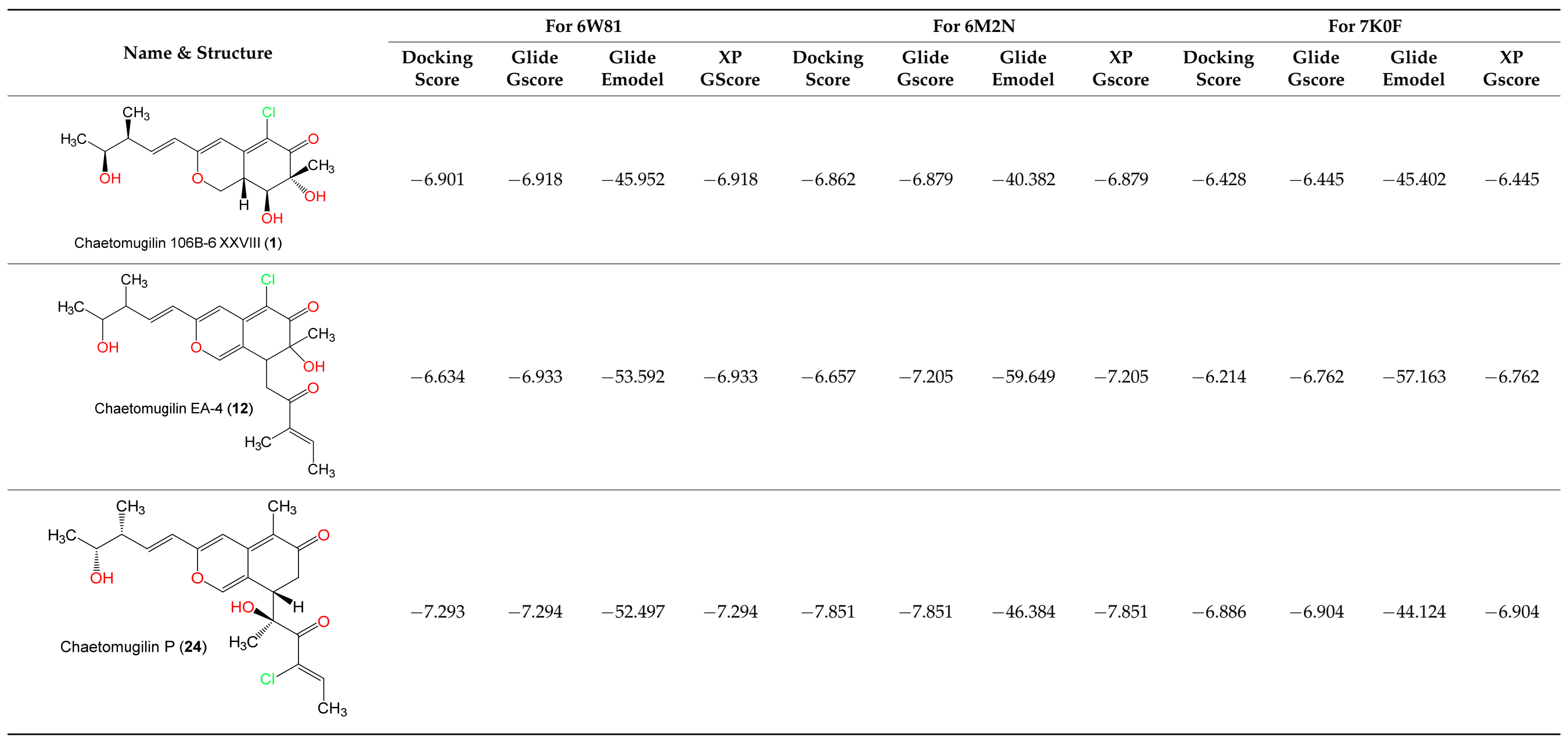


Table 3. Cont.

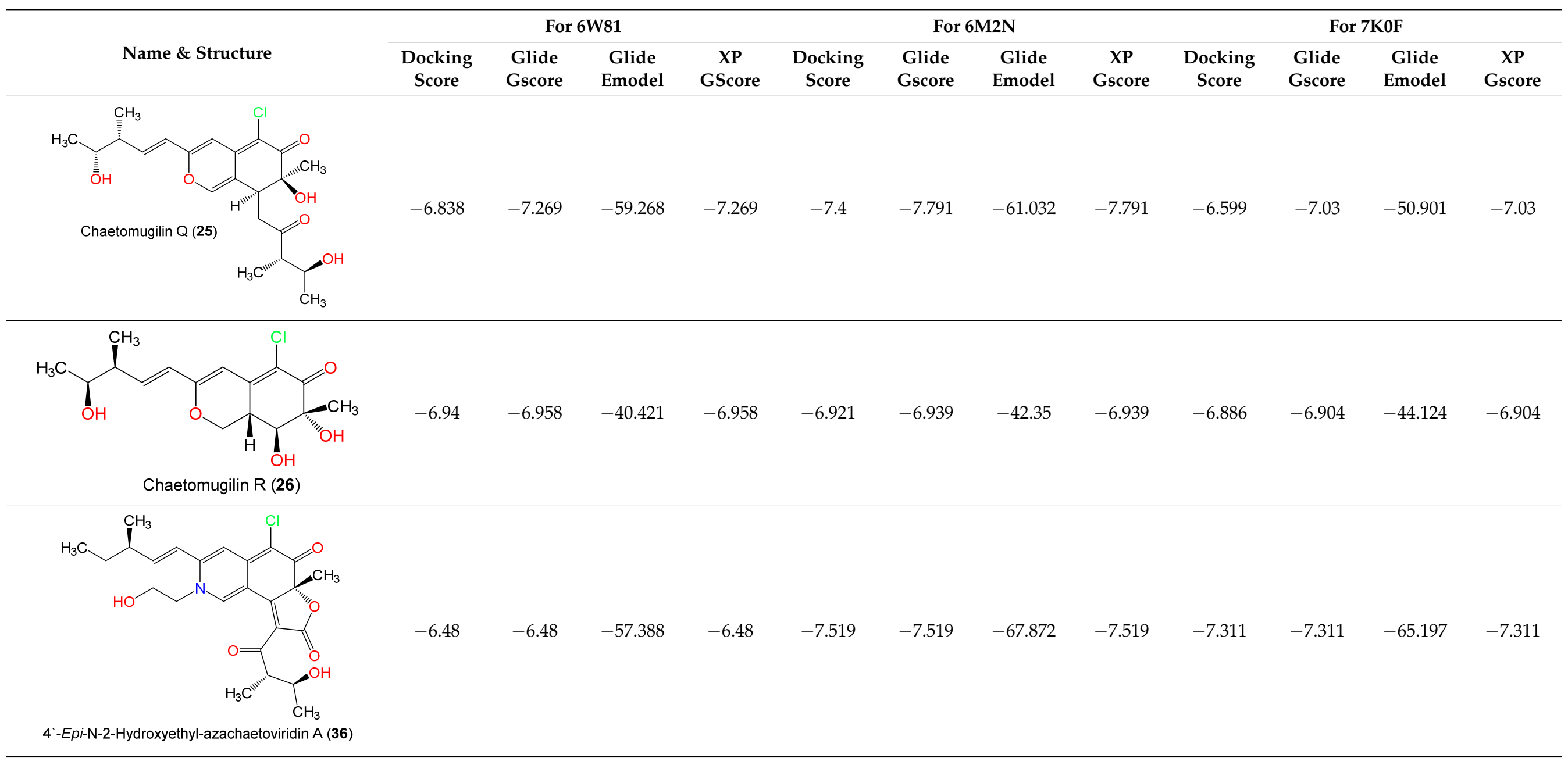


Table 3. Cont.

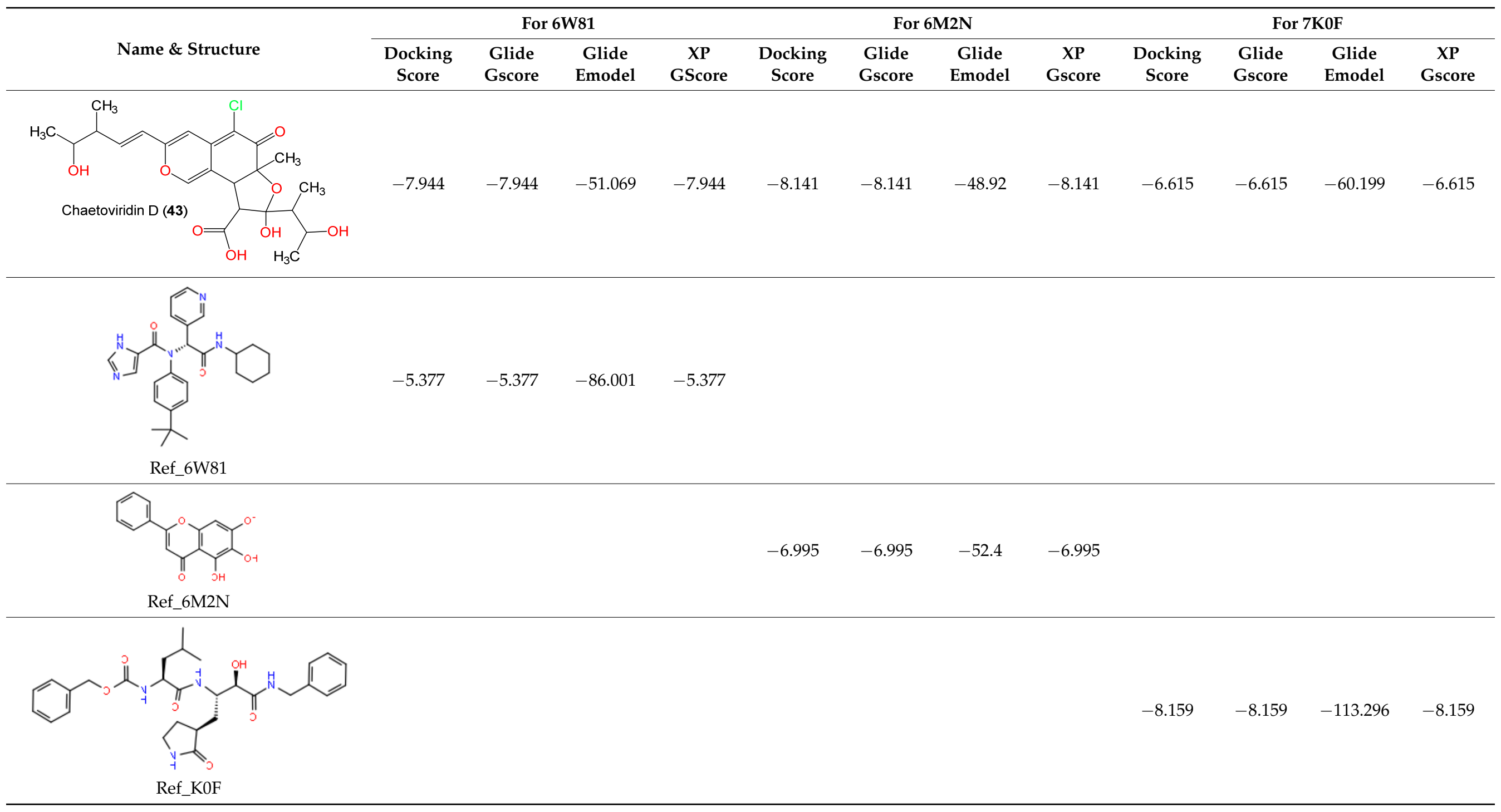


Table 4. In silico screening results of the reported chaetomugilins and chaetoviridins against SARSCoV-2 3CL protease (PDB: 6W81).

\begin{tabular}{|c|c|c|c|c|}
\hline Fungal Chaetomugilins and Chaetoviridins & Docking Score & Glide Gscore & Glide Emodel & XP Gscore \\
\hline Chaetovirdin D (43) & -7.944 & -7.944 & -51.069 & -7.944 \\
\hline Chaetomugilin $\mathrm{P}(\mathbf{2 4})$ & -7.293 & -7.294 & -52.497 & -7.294 \\
\hline N-Glutrylchaetovirdin A (35) & -7.101 & -7.101 & -66.739 & -7.101 \\
\hline Chaetovirdin B (37) & -6.959 & -6.959 & -37.203 & -6.959 \\
\hline Chaetomugilin R (26) & -6.94 & -6.958 & -40.421 & -6.958 \\
\hline Chaetomugilin 106B-6 XXVIII (1) & -6.901 & -6.918 & -45.952 & -6.918 \\
\hline N-Glutarylchaetovirdin C (42) & -6.881 & -6.881 & -60.87 & -6.881 \\
\hline Chaetomugilin Q (25) & -6.838 & -7.269 & -59.268 & -7.269 \\
\hline Chaetomugilin EA-4 (12) & -6.634 & -6.933 & -53.592 & -6.933 \\
\hline $7,4^{\prime}, 5^{\prime}$-Tris-Epi-chaetovirdin A $(34)$ & -6.519 & -6.519 & -56.68 & -6.519 \\
\hline $4^{\prime}$-Epi-N-2-Hydroxyethyl-azachaetovirdin A (36) & -6.48 & -6.48 & -57.388 & -6.48 \\
\hline Chaetomugilin T (26) & -6.407 & -6.407 & -45.87 & -6.407 \\
\hline Chaetovirdin J (53) & -6.295 & -6.673 & -54.688 & -6.673 \\
\hline $12 \beta-$ Hydroxychaetovirdin C (41) & -6.074 & -6.08 & -54.03 & -6.08 \\
\hline Chaetomugilin M (21) & -5.985 & -6.143 & -53.589 & -6.143 \\
\hline Chaetovirdin $G(50)$ & -5.925 & -5.925 & -50.419 & -5.925 \\
\hline Chaetomugilin I (16) & -5.919 & -6.467 & -53.609 & -6.467 \\
\hline N-2-Butyric-azochaetovirdin E (46) & -5.862 & -5.863 & -58.955 & -5.863 \\
\hline 11-Epi-chaetomugilin A (4) & -5.771 & -5.774 & -45.965 & -5.774 \\
\hline Chaetovirdin C (40) & -5.752 & -5.752 & -48.221 & -5.752 \\
\hline Chaetomugilin H (15) & -5.741 & -5.741 & -40.295 & -5.741 \\
\hline N-Glutarylchaetovirdin B (39) & -5.733 & -5.735 & -57.615 & -5.735 \\
\hline Chaetomugilin U (30) & -5.622 & -5.622 & -40.195 & -5.622 \\
\hline $4^{\prime}$-Epi-chaetovirdin A (5) & -5.561 & -5.561 & -47.473 & -5.561 \\
\hline Chaetomugilin S (27) & -5.539 & -5.539 & -45.867 & -5.539 \\
\hline $4^{\prime}, 5^{\prime}$-Bis-chaetovirdin A (33) & -5.507 & -5.507 & -48.38 & -5.507 \\
\hline Chaetovirdin A (31) & -5.493 & -5.493 & -51.732 & -5.493 \\
\hline Ref_6W81 & -5.377 & -5.377 & -86.001 & -5.377 \\
\hline Chaetovirdin I (52) & -5.354 & -5.419 & -42.206 & -5.419 \\
\hline Seco-Chaetomugilin D (9) & -5.345 & -5.347 & -45.22 & -5.347 \\
\hline Chaetomugilin L (20) & -5.25 & -5.25 & -39.261 & -5.25 \\
\hline Chaetomugilin B (6) & -5.248 & -5.248 & -41.211 & -5.248 \\
\hline Chaetomugilin A (2) & -5.241 & -5.243 & -44.852 & -5.243 \\
\hline 11-Epi-chaetomugilin I (17) & -5.099 & -5.647 & -54.765 & -5.647 \\
\hline Chaetomugilin D (8) & -5.093 & -5.095 & -41.171 & -5.095 \\
\hline 7-Epi-chaetovirdin E (45) & -5.089 & -5.089 & -40.702 & -5.089 \\
\hline Chaetovirdin K (54) & -4.987 & -4.989 & -45.166 & -4.989 \\
\hline Chaetomugilin N (22) & -4.977 & -4.977 & -3454.68 & -4.977 \\
\hline Seco-chaetomugilin A (3) & -4.926 & -4.928 & -54.168 & -4.928 \\
\hline Chaetomugilin K (19) & -4.903 & -4.903 & -38.494 & -4.903 \\
\hline Chaetovirdin H (51) & -4.898 & -4.898 & -51.406 & -4.898 \\
\hline $4^{\prime}$-Epi-chaetovirdin F (X48) & -4.873 & -4.873 & -47.708 & -4.873 \\
\hline Chaetovirdin B (38) & -4.792 & -4.794 & -42.458 & -4.794 \\
\hline Chaetovirdin E (44) & -4.61 & -4.61 & -48.663 & -4.61 \\
\hline Chaetomugilin G (14) & -4.516 & -4.516 & -50.66 & -4.516 \\
\hline Chaetomugilin S (28) & -4.391 & -4.393 & -48.292 & -4.393 \\
\hline Chaetomugilin F (13) & -4.28 & -4.28 & -40.132 & -4.28 \\
\hline Chaetovirdin F (47) & -4.271 & -4.271 & -50.793 & -4.271 \\
\hline Epi-chaetomugilin D (10) & -4.137 & -4.139 & -36.43 & -4.139 \\
\hline Chaetomugilin E (11) & -3.597 & -3.597 & -40.424 & -3.597 \\
\hline Chaetomugilin C (7) & -3.509 & -3.509 & -44.435 & -3.509 \\
\hline Chaetovirdin G (49) & -3.492 & -3.492 & -48.133 & -3.492 \\
\hline
\end{tabular}


Table 5. In silico screening results of the reported chaetomugilins and chaetoviridins against SARSCoV-2 3CL protease (PDB: 6M2N).

\begin{tabular}{|c|c|c|c|c|}
\hline Fungal Chaetomugilins and Chaetoviridins & Docking Score & Glide Gscore & Glide Emodel & XP Gscore \\
\hline Chaetovirdin D (43) & -8.141 & -8.141 & -48.92 & -8.141 \\
\hline Chaetovirdin B (37) & -7.851 & -7.851 & -46.384 & -7.851 \\
\hline 4'-Epi-N-2-Hydroxyethyl-azachaetovirdin A (36) & -7.519 & -7.519 & -67.872 & -7.519 \\
\hline Chaetomugilin Q (25) & -7.4 & -7.791 & -61.032 & -7.791 \\
\hline $12 \beta$-Hydroxychaetovirdin C (41) & -7.045 & -7.051 & -58.607 & -7.051 \\
\hline Ref_6M2N & -6.995 & -6.995 & -52.4 & -6.995 \\
\hline Chaetomugilin R (26) & -6.921 & -6.939 & -42.35 & -6.939 \\
\hline Chaetomugilin P (24) & -6.9 & -6.901 & -48.711 & -6.901 \\
\hline Chaetomugilin 106B-6 XXVIII (1) & -6.862 & -6.879 & -40.382 & -6.879 \\
\hline Chaetomugilin S (27) & -6.785 & -6.785 & -50.138 & -6.785 \\
\hline Chaetomugilin EA-4 (12) & -6.657 & -7.205 & -59.649 & -7.205 \\
\hline Chaetomugilin $\mathrm{M}(\mathbf{2 1})$ & -6.655 & -6.813 & -56.59 & -6.813 \\
\hline $7,4^{\prime}, 5^{\prime}$-Tris-Epi-chaetovirdin A (34) & -6.44 & -6.44 & -55.163 & -6.44 \\
\hline Chaetovirdin I (52) & -6.439 & -6.504 & -53.296 & -6.504 \\
\hline Chaetovirdin J (53) & -6.362 & -6.741 & -54.219 & -6.741 \\
\hline Chaetovirdin G (50) & -6.312 & -6.312 & -48.248 & -6.312 \\
\hline Chaetomugilin G (49) & -6.272 & -6.272 & -56.423 & -6.272 \\
\hline Chaetovirdin C (40) & -6.219 & -6.219 & -59.734 & -6.219 \\
\hline N-Glutarylchaetovirdin B (39) & -6.178 & -6.179 & -53.868 & -6.179 \\
\hline 11-Epi-chaetomugilin I (17) & -6.077 & -6.625 & -61.469 & -6.625 \\
\hline Chaetovirdin $\mathrm{H}(\mathbf{5 1})$ & -6.071 & -6.071 & -45.006 & -6.071 \\
\hline Chaetovirdin A (31) & -5.93 & -5.93 & -47.164 & -5.93 \\
\hline $4^{\prime}, 5^{\prime}$-Bis-chaetovirdin A (33) & -5.896 & -5.896 & -46.971 & -5.896 \\
\hline Seco-chaetomugilin A (3) & -5.89 & -5.892 & -44.484 & -5.892 \\
\hline Chaetovirdin E (44) & -5.871 & -5.871 & -48.647 & -5.871 \\
\hline Chaetomugilin I (16) & -5.87 & -6.418 & -55.891 & -6.418 \\
\hline Chaetovirdin F (47) & -5.866 & -5.866 & -54.419 & -5.866 \\
\hline $4^{\prime}$-Epi-chaetovirdin F (48) & -5.859 & -5.859 & -51.775 & -5.859 \\
\hline Chaetomugilin F (13) & -5.759 & -5.759 & -43.54 & -5.759 \\
\hline N-2-Butyric-azochaetovirdin E (46) & -5.453 & -5.454 & -54.064 & -5.454 \\
\hline N-Glutrylchaetovirdin A (35) & -5.405 & -5.405 & -59.359 & -5.405 \\
\hline Seco-Chaetomugilin D (9) & -5.376 & -5.379 & -41.076 & -5.379 \\
\hline Chaetomugilin H (15) & -5.293 & -5.293 & -52.628 & -5.293 \\
\hline Chaetovirdin B (38) & -5.217 & -5.219 & -38.105 & -5.219 \\
\hline 11-Epi-chaetomugilin A (4) & -5.166 & -5.168 & -41.465 & -5.168 \\
\hline Chaetovirdin K (54) & -5.11 & -5.113 & -38.902 & -5.113 \\
\hline Chaetomugilin A (2) & -5.09 & -5.092 & -36.177 & -5.092 \\
\hline Chaetomugilin T (29) & -5.012 & -5.012 & -39.774 & -5.012 \\
\hline Chaetomugilin C (7) & -4.987 & -4.987 & -44.839 & -4.987 \\
\hline $4^{\prime}$-Epi-chaetovirdin A (32) & -4.905 & -4.905 & -58.103 & -4.905 \\
\hline Chaetomugilin S (28) & -4.627 & -4.629 & -36.966 & -4.629 \\
\hline Chaetovirdin G (49) & -4.612 & -4.612 & -42.176 & -4.612 \\
\hline 7-Epi-chaetovirdin E (45) & -4.485 & -4.485 & -51.758 & -4.485 \\
\hline Epi-chaetomugilin D (10) & -4.341 & -4.343 & -31.912 & -4.343 \\
\hline Chaetomugilin U (30) & -4.248 & -4.249 & -40.187 & -4.249 \\
\hline Chaetomugilin O (23) & -4.208 & -4.208 & -1767.6 & -4.208 \\
\hline Chaetomugilin N (22) & -4.07 & -4.07 & -31.442 & -4.07 \\
\hline Chaetomugilin B (6) & -4.068 & -4.068 & -41.99 & -4.068 \\
\hline Chaetomugilin K (19) & -3.867 & -3.867 & -34.284 & -3.867 \\
\hline Chaetomugilin D (8) & -3.667 & -3.669 & -35.613 & -3.669 \\
\hline Chaetomugilin L (20) & -3.622 & -3.622 & -38.371 & -3.622 \\
\hline Chaetomugilin E (11) & -3.506 & -3.506 & -41.116 & -3.506 \\
\hline N-Glutarylchaetovirdin C (42) & -1.412 & -1.412 & -62.951 & -1.412 \\
\hline
\end{tabular}


Table 6. In silico screening results of the reported chaetomugilins and chaetoviridins against SARSCoV-2 3CL protease (PDB: 7K0F).

\begin{tabular}{|c|c|c|c|c|}
\hline Fungal Chaetomugilins and Chaetoviridins & Docking Score & Glide Gscore & Glide Emodel & XP Gscore \\
\hline Ref_7K0F & -8.159 & -8.159 & -113.296 & -8.159 \\
\hline $4^{\prime}$-Epi-N-2-Hydroxyethyl-azachaetovirdin A (36) & -7.311 & -7.311 & -65.197 & -7.311 \\
\hline Chaetomugilin R (26) & -6.886 & -6.904 & -44.124 & -6.904 \\
\hline Chaetovirdin D (43) & -6.615 & -6.615 & -60.199 & -6.615 \\
\hline Chaetomugilin Q (25) & -6.599 & -7.03 & -50.901 & -7.03 \\
\hline Chaetomugilin 106B-6 XXVIII (1) & -6.428 & -6.445 & -45.402 & -6.445 \\
\hline Chaetovirdin I (52) & -6.381 & -6.446 & -60.443 & -6.446 \\
\hline Chaetomugilin EA-4 (12) & -6.214 & -6.762 & -57.163 & -6.762 \\
\hline Seco-chaetomugilin A (3) & -6.164 & -6.166 & -54.533 & -6.166 \\
\hline Chaetomugilin T (29) & -6.003 & -6.003 & -44.936 & -6.003 \\
\hline Chaetomugilin A (2) & -5.937 & -5.939 & -49.214 & -5.939 \\
\hline $4^{\prime}$-Epi-chaetovirdin F (48) & -5.781 & -5.781 & -53.249 & -5.781 \\
\hline Chaetovirdin G (50) & -5.73 & -5.73 & -46.915 & -5.73 \\
\hline 11-Epi-chaetomugilin A (4) & -5.634 & -5.636 & -45.502 & -5.636 \\
\hline Chaetomugilin M (21) & -5.56 & -5.718 & -41.62 & -5.718 \\
\hline Chaetovirdin K (54) & -5.521 & -5.523 & -43.983 & -5.523 \\
\hline Chaetovirdin H (51) & -5.487 & -5.487 & -53.211 & -5.487 \\
\hline Chaetovirdin J (53) & -5.475 & -5.853 & -56.559 & -5.853 \\
\hline Chaetomugilin D (8) & -5.457 & -5.459 & -40.705 & -5.459 \\
\hline Chaetomugilin S (28) & -5.409 & -5.411 & -37.945 & -5.411 \\
\hline Chaetovirdin A (31) & -5.356 & -5.356 & -55.812 & -5.356 \\
\hline Chaetomugilin J (18) & -5.262 & -5.758 & -44.768 & -5.758 \\
\hline Chaetovirdin F (47) & -5.214 & -5.214 & -51.016 & -5.214 \\
\hline Chaetovirdin E (44) & -5.207 & -5.207 & -57.958 & -5.207 \\
\hline Chaetomugilin U (30) & -5.17 & -5.17 & -39.245 & -5.17 \\
\hline Chaetomugilin G (14) & -5.154 & -5.154 & -52.872 & -5.154 \\
\hline $4^{\prime}, 5^{\prime}$-Bis-chaetovirdin A (33) & -5.147 & -5.147 & -56.898 & -5.147 \\
\hline Chaetomugilin H (15) & -5.144 & -5.144 & -51.211 & -5.144 \\
\hline Chaetovirdin C (40) & -5.119 & -5.119 & -51.53 & -5.119 \\
\hline Epi-chaetomugilin D (10) & -5.11 & -5.112 & -41.394 & -5.112 \\
\hline Chaetomugilin P (24) & -5.083 & -5.085 & -47.434 & -5.085 \\
\hline Chaetomugilin S (27) & -5.005 & -5.005 & -53.874 & -5.005 \\
\hline N-2-Butyric-azochaetovirdin E (46) & -4.926 & -4.927 & -58.939 & -4.927 \\
\hline $7,4^{\prime}, 5^{\prime}$-Tris-Epi-chaetovirdin A (34) & -4.883 & -4.883 & -50.65 & -4.883 \\
\hline 11-Epi-chaetomugilin I (17) & -4.785 & -5.084 & -57.348 & -5.084 \\
\hline Chaetomugilin B (6) & -4.732 & -4.732 & -44.687 & -4.732 \\
\hline Chaetomugilin I (16) & -4.534 & -4.834 & -53.036 & -4.834 \\
\hline Chaetomugilin C (7) & -4.469 & -4.469 & -45.833 & -4.469 \\
\hline $12 \beta$-Hydroxychaetovirdin C (41) & -4.352 & -4.357 & -51.515 & -4.357 \\
\hline Chaetomugilin E (11) & -4.268 & -4.268 & -39.678 & -4.268 \\
\hline N-Glutarylchaetovirdin C (42) & -4.247 & -4.247 & -59.534 & -4.247 \\
\hline Chaetomugilin K (19) & -4.245 & -4.245 & -47.953 & -4.245 \\
\hline Chaetovirdin G (59) & -4.218 & -4.218 & -46.464 & -4.218 \\
\hline Seco-Chaetomugilin D (9) & -4.205 & -4.207 & -48.935 & -4.207 \\
\hline N-Glutrylchaetovirdin A (39) & -4.171 & -4.171 & -55.598 & -4.171 \\
\hline Chaetomugilin F (13) & -3.88 & -3.88 & -42.311 & -3.88 \\
\hline Chaetomugilin R (26) & -3.808 & -5.942 & -41.217 & -5.942 \\
\hline 7-Epi-chaetovirdin E (45) & -3.699 & -3.699 & -46.38 & -3.699 \\
\hline Chaetomugilin L (20) & -3.612 & -3.612 & -43.218 & -3.612 \\
\hline $4^{\prime}$-Epi-chaetovirdin A (32) & -3.552 & -3.552 & -58.379 & -3.552 \\
\hline Chaetomugilin $\mathrm{O}(\mathbf{2 3})$ & -2.951 & -2.951 & 31.679 & -2.951 \\
\hline Chaetovirdin B (38) & -2.642 & -2.644 & -45.702 & -2.644 \\
\hline Chaetomugilin N (22) & -0.921 & -0.921 & -3319.51 & -0.921 \\
\hline N-Glutarylchaetovirdin B (39) & -0.451 & -0.453 & -57.257 & -0.453 \\
\hline
\end{tabular}


Table 7. In silico predicted ADME properties of the selected fungal chaetomugilins and chaetoviridins.

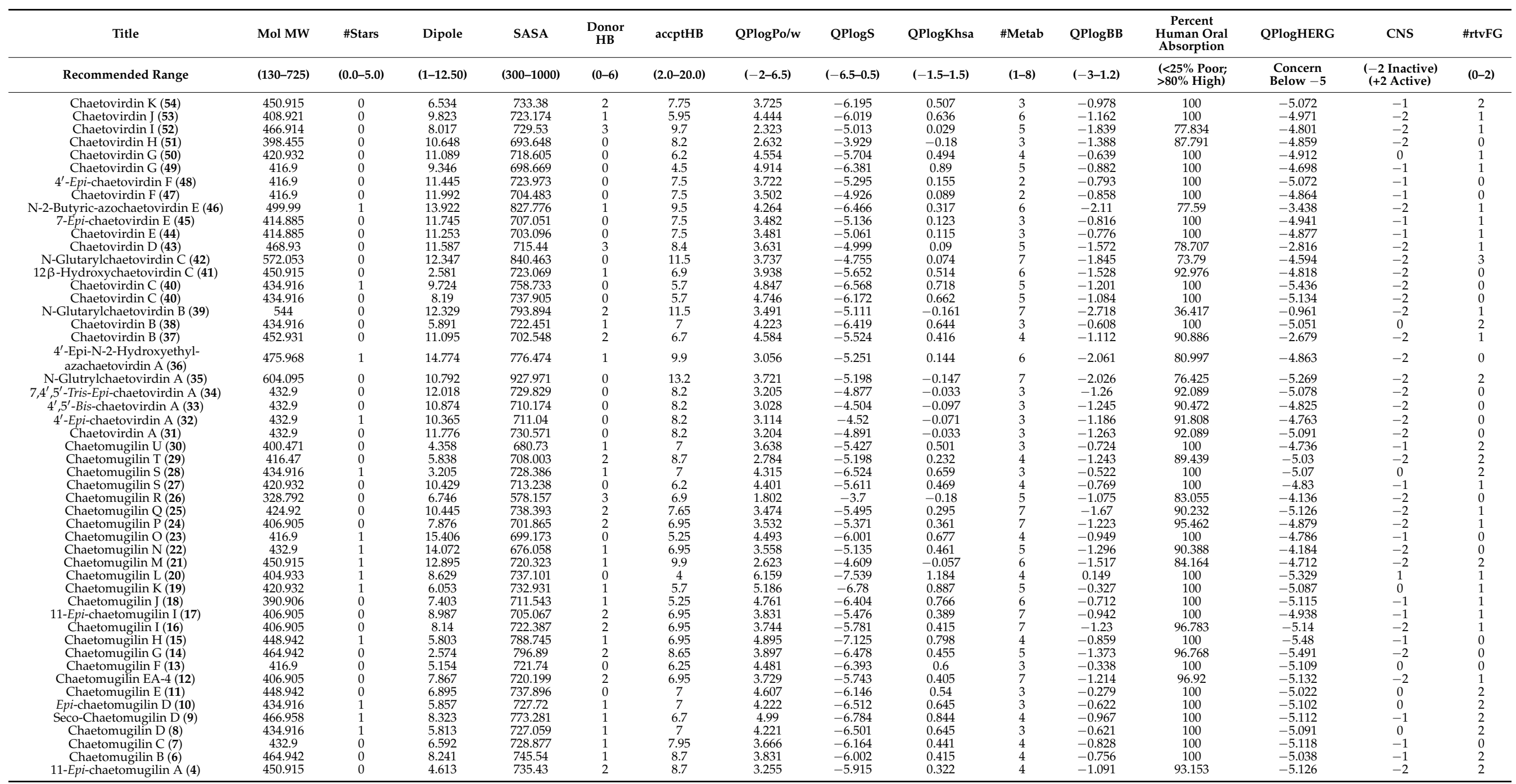


Table 7. Cont.

\begin{tabular}{|c|c|c|c|c|c|c|c|c|c|c|c|c|c|c|c|}
\hline Title & Mol MW & \#Stars & Dipole & SASA & $\begin{array}{c}\text { Donor } \\
\mathrm{HB}\end{array}$ & accptHB & QPlogPo/w & QPlogS & QPlogKhsa & \#Metab & QPlogBB & $\begin{array}{c}\text { Percent } \\
\text { Human Oral } \\
\text { Absorption }\end{array}$ & QPlogHERG & CNS & \#rtvFG \\
\hline Recommended Range & $(130-725)$ & $(0.0-5.0)$ & $(1-12.50)$ & $(300-1000)$ & $(0-6)$ & $(2.0-20.0)$ & $(-2-6.5)$ & $(-6.5-0.5)$ & $(-1.5-1.5)$ & $(1-8)$ & $(-3-1.2)$ & $\begin{array}{l}\text { (<25\% Poor; } \\
>80 \% \text { High) }\end{array}$ & $\begin{array}{l}\text { Concern } \\
\text { Below -5 }\end{array}$ & $\begin{array}{c}\text { (-2 Inactive) } \\
\text { (+2 Active) }\end{array}$ & $(0-2)$ \\
\hline $\begin{array}{l}\text { Seco-chaetomugilin A (3) } \\
\text { Cheetomugilin A (2) }\end{array}$ & $\begin{array}{l}482.957 \\
450.915\end{array}$ & $\begin{array}{l}0 \\
0\end{array}$ & $\begin{array}{l}7.988 \\
4.464\end{array}$ & $\begin{array}{l}774.455 \\
734.849\end{array}$ & $\begin{array}{l}2 \\
2\end{array}$ & $\begin{array}{l}8.4 \\
8.7\end{array}$ & $\begin{array}{l}3.951 \\
3.266\end{array}$ & $\begin{array}{l}-6.021 \\
-5.905\end{array}$ & $\begin{array}{l}0.479 \\
0.335\end{array}$ & $\begin{array}{l}5 \\
4\end{array}$ & $\begin{array}{l}-1.427 \\
-1.106\end{array}$ & $\begin{array}{l}95.73 \\
92.834\end{array}$ & $\begin{array}{l}-5.066 \\
-5.084\end{array}$ & $\begin{array}{l}-2 \\
-2\end{array}$ & $\frac{2}{2}$ \\
\hline Chaetomugilin 106B-6 XXVIII (1) & 328.792 & 0 & 6.746 & 578.786 & 3 & 6.9 & 1.79 & -3.71 & -0.186 & 5 & -1.081 & 82.981 & -4.167 & -2 & 0 \\
\hline
\end{tabular}

\#Stars: Number of property or descriptor values that fall outside the $95 \%$ range of similar values for known drugs. A large number of stars suggests that a molecule is less drug-like than molecules with few stars. Dipole: Computed dipole moment of the molecule. SASA: Total solvent accessible surface area (SASA) in square angstroms using a probe with a $1.4 \AA$ radius. Donor H-bond: Estimated number of hydrogen bonds that the solute would donate to water molecules in an aqueous solution. Acceptor H-bond: Estimated number of hydrogen bonds that the solute would accept from water molecules in an aqueous solution. QPlogPo/w: Predicted octanol/water partition coefficient. QPlogS: Predicted aqueous solubility, log S. QPlogkhsa: Prediction of binding to human serum albumin. No. of Metabolites: Number of likely metabolic reactions. QplogBB: Predicted brain/blood partition coefficient. \% Human Oral Absorption: Predicted human oral absorption on 0 to $100 \%$ scale. QPlogHERG: Predicted $\mathrm{IC}_{50}$ value for blockage of HERG K ${ }^{+}$channels. CNS: Predicted central nervous system activity on a -2 (inactive) to +2 (active) scale. \#rtvFG: Number of reactive functional groups; the specific groups are listed in the jobname out file. The presence of these groups can lead to false positives in HTS assays and to decomposition, reactivity, or toxicity problems in vivo. 


\subsubsection{Preparations of Ligands and Proteins}

By using LigPrep, the conversion of 2D structures to 3D, tautomerization, and ionization yielded 254 minimized 3D structures. The minimized 3D structures were used for docking with the crystal structure of the 3CL hydrolase (Mpro). Preparation of the viral protease (6M2N, 6W81, and 7K0f) by the Protein Preparation Wizard tool optimized the H-bonding network and minimized the geometry. Assurance of assigning the proper formal charges and force field treatments was achieved by adding missing hydrogens and correct ionization states (Figure 9).
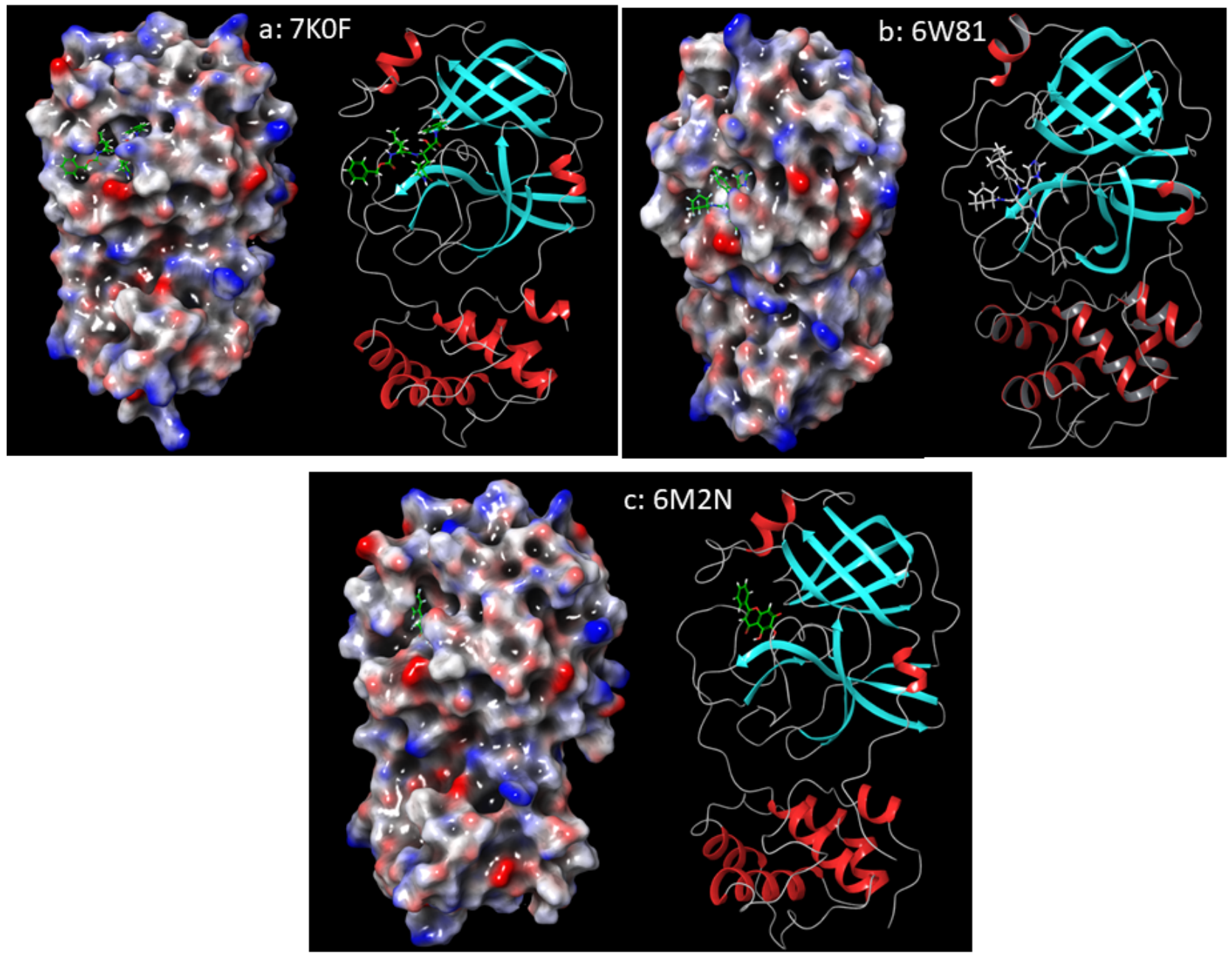

Figure 9. The 3CL pro-hydrolase prepared via Protein Preparation Wizard, Maestro. H-bonding network optimized and geometry minimized structures of 2019-nCOV 3CL hydrolase (Mpro)—PDB ID: (a) 7K0F, (b) 6W81, (c), and 6M2N. Represented as 3D molecular surfaces and ribbon structures.

\subsubsection{Molecular Docking Studies}

After defining the grid box in the prepared viral protease via the Receptor Grid Generation tool of Glide in Maestro, the prepared 3D molecular structures were docked into the co-crystallized inhibitor binding site of the viral protease. Table 3 shows the results of the top-score docked ligands chosen based on the most negative docking scores. These scores represent the best bound ligand conformations and relative binding affinities. Chaetovirdin D (43) exhibited the highly negative docking scores of $-7.944,-8.141$, and $-6.615 \mathrm{kcal} / \mathrm{mol}$, complexed with $6 \mathrm{M} 2 \mathrm{~N}, 6 \mathrm{~W} 81$, and $7 \mathrm{~K} 0 \mathrm{f}$, respectively. The reference inhibitor exhibited the following scores: $-5.377,-6.995$, and $-8.159 \mathrm{kcal} / \mathrm{mol}$, complexed with $6 \mathrm{M} 2 \mathrm{~N}, 6 \mathrm{~W} 81$, and 7K0f, respectively. 
The docking analysis was updated by re-docking the selected chaetomugilins and chaetoviridins with the three crystal structures of the 3CL pro-hydrolase (PDB ID: 6M2N, $6 \mathrm{~W} 81$, and $7 \mathrm{~K} 0 \mathrm{f}$ ) at variable resolutions, 2.20, 1.55, and 1.65A, respectively. Analysis of the docking scores of these compounds with the inhibitor binding sites of 2019-nCoV main protease (PDB ID: 6M2N, 6W81, and 7K0f) revealed different docking scores, and hence binding affinities (Tables 4-6). These differences can be attributed to the differences in the grid formation due to the presence of different inhibitors in the binding sites of the three crystal structures.

Analysis of the docking of 43 revealed that it interacted through hydrogen bonds (Figure 10) with the binding site residues of SARS-CoV-2 main protease (6W81). The binding site residues Asn141, Gly142, and Thr189 of the viral protease exhibited hydrogen bonding with the various hydroxyl groups of 43 . Additionally, 43 interacted through hydrogen bonds (Figure 11) with the binding site residues of SARS-CoV-2 main protease (6M2N). The binding site residues Asn142 and Thr190 of the viral protease showed hydrogen bonding with the various hydroxyl groups of 43 . Its interactions through hydrogen bonds with the binding site residues of SARS-CoV-2 main protease (7K0F) are shown in Figure 12. The binding site residues Thr24, His41, Gly143, His 164, and Glu166 of the viral protease displayed H-bonding with the various hydroxyl groups of 43 .
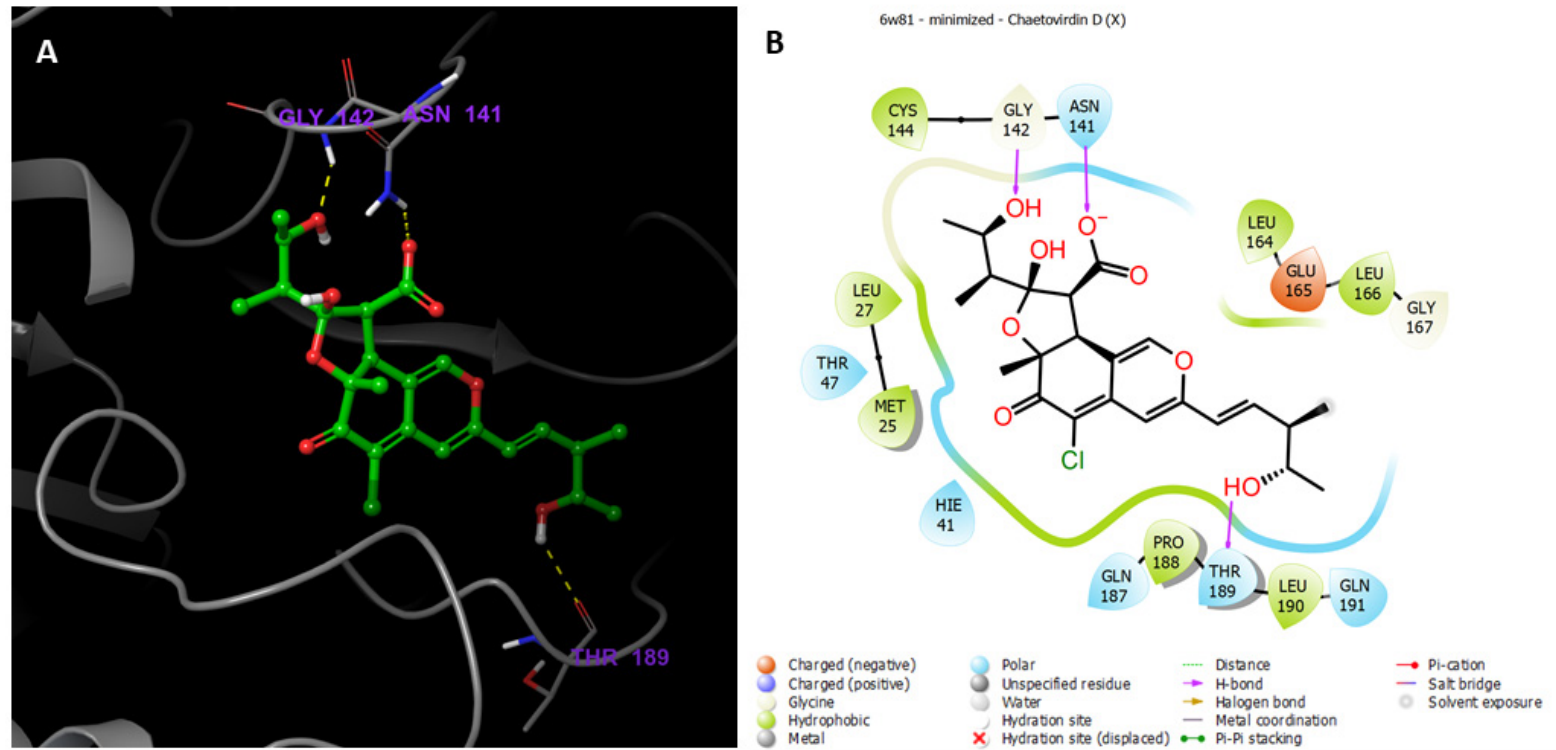

Figure 10. (A) Putative binding mode of chaetovirdin D in the binding site of 2019-nCOV 3CL hydrolase (Mpro), PDB: 6W81. Chaetovirdin D is displayed as green sticks. The amino acids of the binding site are represented as grey sticks, and H-bonds are represented as yellow dotted lines. (B) 2D depiction of the ligand-protein interactions.

\subsubsection{In Silico ADMET Properties of Selected Ligands}

Fifty-three compounds from among the reported fungal chaetomugilins and chaetoviridins were selected and processed using LigPrep of the Schrodinger suite (Schrödinger Release 2021-4: LigPrep, Schrödinger, LLC, New York, NY, USA, 2021; making 3D models with ionization states at $\mathrm{pH} 7.0 \pm 0.2$ generated by the OPLS3 force field. The QikProp module of the Schrodinger suite (Schrödinger Release 2021-4: QikProp, Schrödinger, LLC, New York, NY, USA, 2021; was used to predict the ADME properties. The predicted ADMET properties are summarized in Table 7. ADMET analysis describes and determines the biological function, drug-likeness, physicochemical characters, and expected toxicity of compounds. This is meant to evaluate the usefulness of the molecules. The examined descriptors, such as drug-likeness, molecular weight, solvent accessible surface area, dipole moment, hydrogen bond acceptors, donor traits, aqueous solubility, octanol/water coefficient, binding to hu- 
man serum albumin, number of likely metabolic reactions, brain-blood partition coefficient, human oral absorption, $\mathrm{IC}_{50}$ value for blockage of HERG $\mathrm{K}^{+}$channels, central nervous system activity, and number of reactive functional groups, were predicted for the reported metabolites. The values obtained for all the compounds are in the recommended ranges.
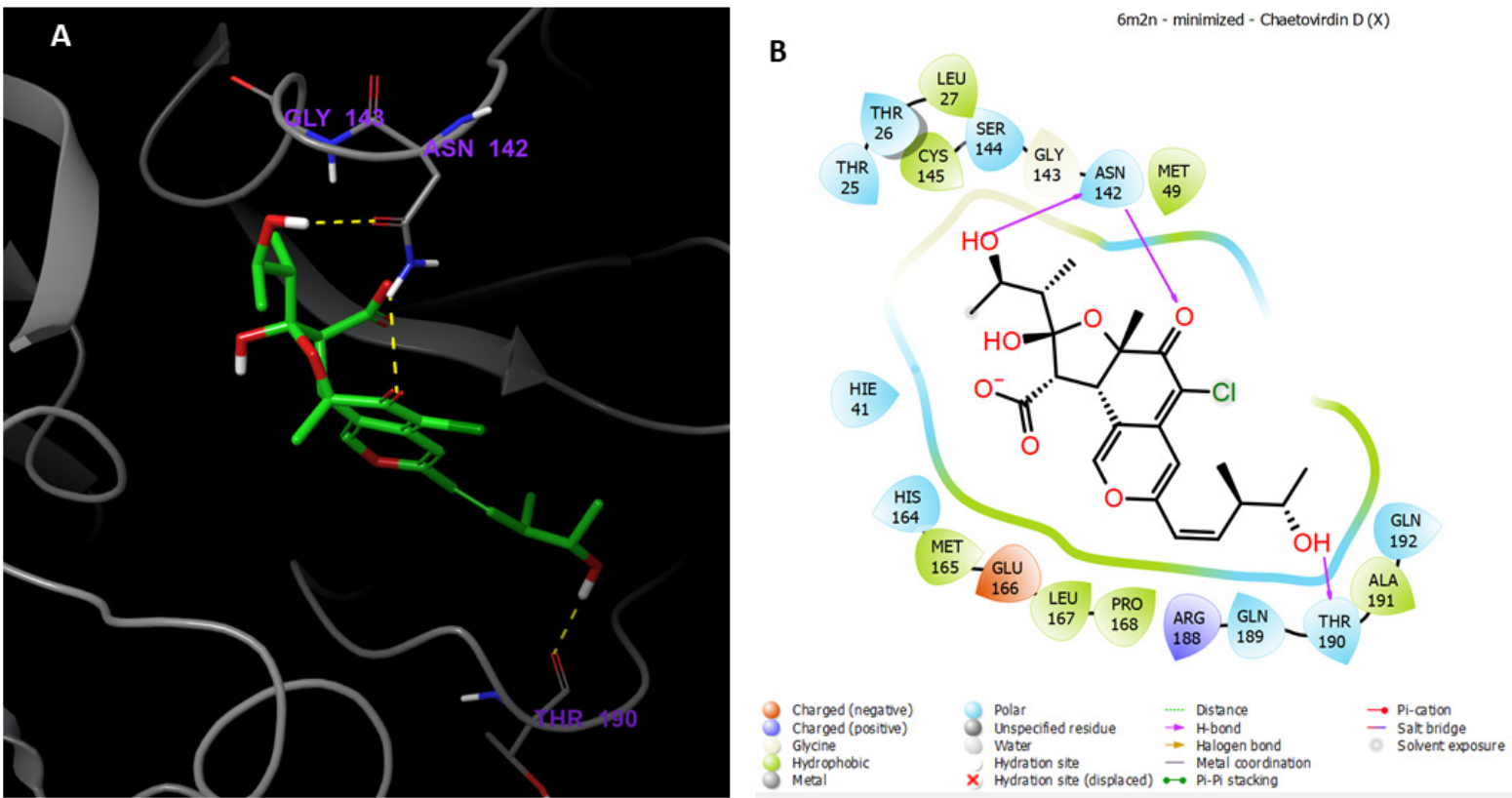

Figure 11. (A) Putative binding mode of Chaetovirdin D in the binding site of 2019-nCOV 3CL hydrolase (Mpro), PDB: 6M2N. Chaetovirdin D is displayed as green sticks. The amino acids of the binding site are represented as grey sticks, and H-bonds are represented as yellow dotted lines. (B) 2D depiction of the ligand-protein interactions.
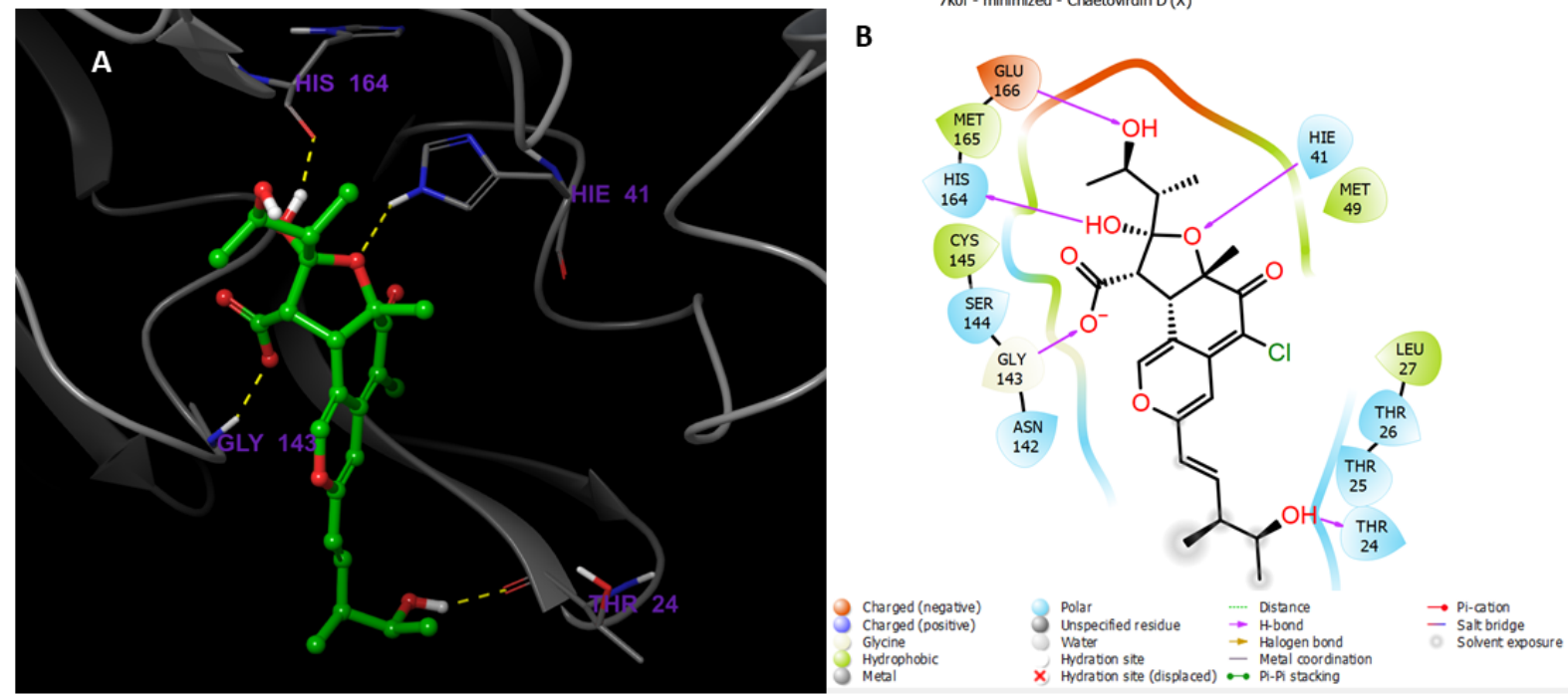

Figure 12. (A) Putative binding mode of Chaetovirdin D in the binding site of 2019-nCOV 3CL hydrolase (Mpro), PDB: 7K0F. Chaetovirdin D is displayed as green sticks. The amino acids of the binding site are represented as grey sticks, and H-bonds are represented as yellow dotted lines. (B) 2D depiction of the ligand-protein interactions. 


\subsubsection{Molecular Dynamics Simulation}

The docking studies took a static view for the binding of each molecule in the active site of the protein. A molecular dynamics (MD) simulation computes the atoms movements over time. By using Desmond software, the stability and frequency of the 43 complex with the proteases-PDB ID 6M2N, 6W81, and 7K0f-were studied. Three MD simulations were run for complexes with $\mathbf{4 3}$ for $100 \mathrm{~ns}$ of simulated time; the complexes' structures were optimized at pH 7.0 \pm 2.0 . Complex stability was checked by analysis of the interaction map and the RMSD (root mean square deviation) plot of the ligand and protein.

The RMSD plots in Figure 13a for the chaetovirdin D-SARS-CoV-2 main protease (PDB ID 6W81) complex, Figure 13b for the chaetovirdin D-SARS-CoV-2 main protease (PDB ID $7 \mathrm{~K} 0 \mathrm{~F}$ ) complex, and Figure 13c for the chaetovirdin D-SARS-CoV-2 main protease (PDB ID $6 \mathrm{M} 2 \mathrm{~N}$ ) indicate that the complexes tended to stabilize during the simulations (100 ns) with respect to a reference frame at time $0 \mathrm{~ns}$. There were slight fluctuations during the simulations, but within the permissible range of $1-3 \AA$; hence, they can be considered non-significant. Since the RMSD plots of $\mathbf{4 3}$ and protein backbone lie over each other, the formation of a stable complex can be inferred.
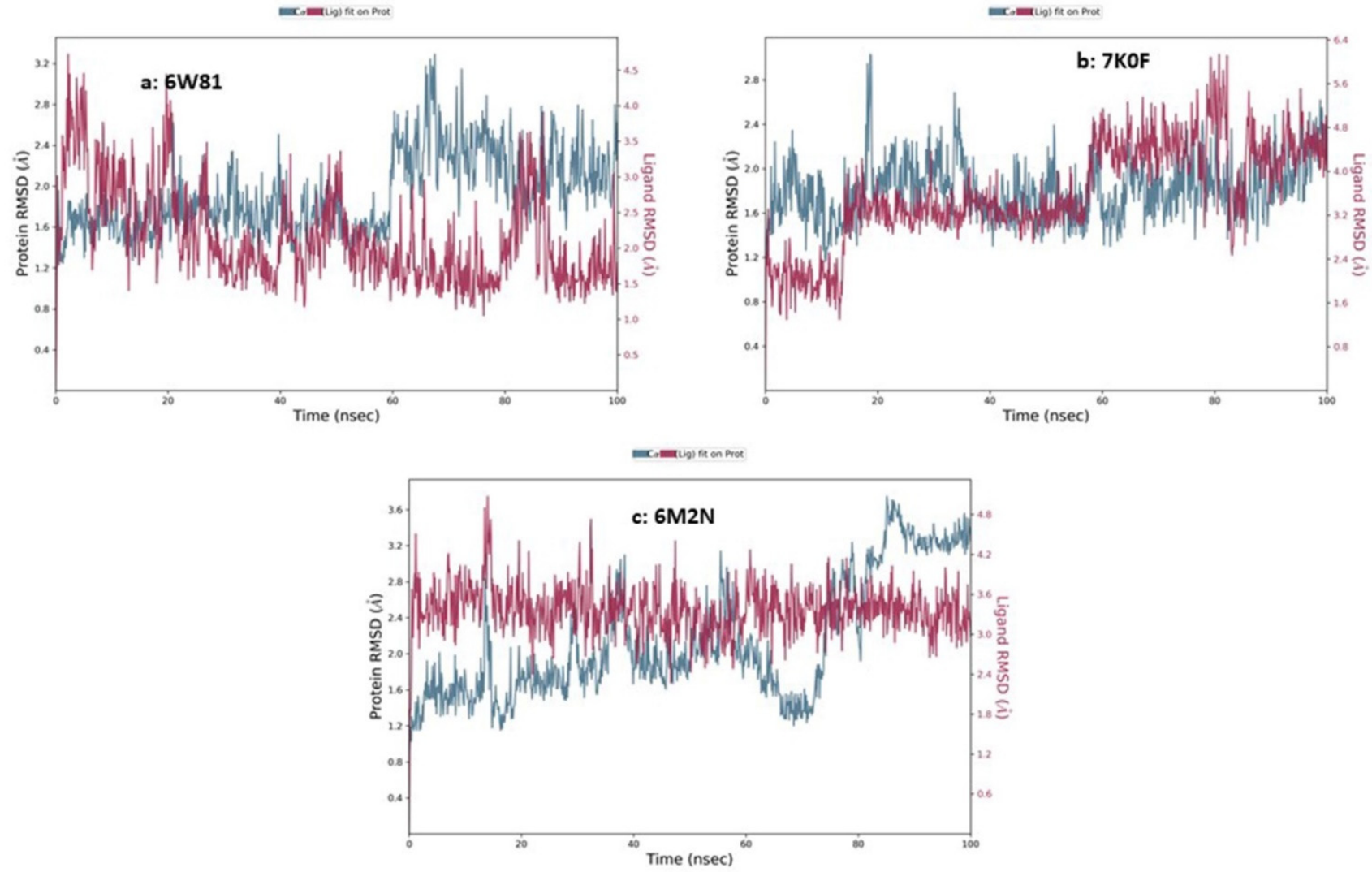

Figure 13. RMSD analysis of MD simulation trajectory. The RMSD plot obtained for (a) chaetovirdin D-SARS-CoV-2 main protease complex (PDB ID 6W81), (b) chaetovirdin D-SARS-CoV-2 main protease complex (PDB ID 7K0F), and (c) chaetovirdin D-SARS-CoV-2 main protease complex (PDB ID 6M2N). The simulated time of 100 ns shows the formation of a stable complex without any significant conformational changes in protein structure.

Figure 14a shows the residue interactions of chaetovirdin D with SARS-CoV-2 main protease (PDB ID 6W81) (the docked poses were retained during the simulation of $100 \mathrm{~ns}$ ) i.e., molecular interactions with His41, Asp141, and Glu165 residues. Moreover, a hydrophobic interaction was also established between the Leu165 and aliphatic hydrocarbons of 43. In Figure 14b, the viral protease (PDB ID 7K0F)-chaetovirdin D contacts over the course of $100 \mathrm{~ns}$ are categorized into water bridges, hydrogen bonds, and hydrophobic 
interactions. The initial docked pose of $\mathbf{4 3}$ shows that the important hydrogen bonds (Thr24, His41, Asn142, Gly143, Ser144, Cys145) did not change during the MD simulation. Hydrogen bonding with residue Cys145 was retained for more than $70 \%$ of the simulation time. Figure $14 \mathrm{c}$ reveals that the viral protease (PDB ID 6M2N)-chaetovirdin D contacts over the course of 100 ns were categorized into water bridges, hydrogen bonds, and hydrophobic interactions. The bar chart shows that hydrogen bonds, hydrophobic contacts, and water bridges prevailed during the course of the simulation. The important hydrogen bonds observed in the initial docked pose of 43 (Thr26, His41, Glu166, Thr190, Gln192) did not change during the MD simulation. Hydrogen bonding with residue Thr26 was retained for more than $5 \%$ of the simulation time.
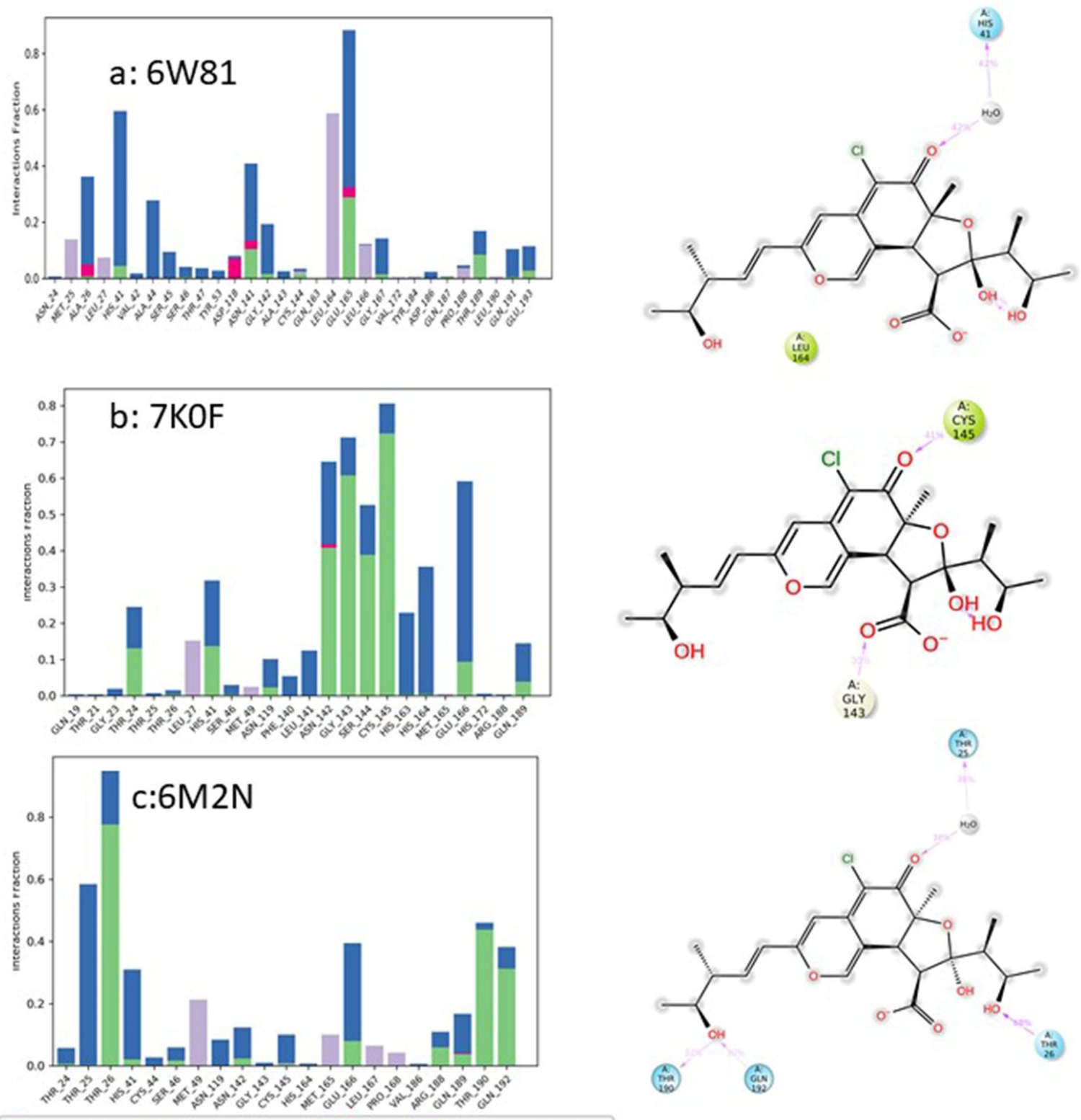

H-bonds $\square$ Hydrophobic $\square$ lonic $\square$ Water bridges

Figure 14. Analysis of molecular interactions and types of contacts with 3CL pro-hydrolase during the MD simulations. Detailed schematic interactions of chaetovirdin D atoms with binding site residues of hydrolase crystal structures (a) 6W81, (b) 7K0F, and (c) 6M2N. Interactions happening during more than $30 \%$ of the simulation is shown. Normalized stacked bar charts show the viral protease binding site residues interacting with chaetovirdin D via hydrogen bonds, hydrophobic and ionic interactions, and water bridges. 


\subsubsection{Materials and Methods}

Preparation of PDB Structures

The three PDB structures (PDB ID: 6M2N, 6W81, and 7K0f) were downloaded from the Protein Data Bank (Protein Data Bank; available online, prepared, and optimized by using "Protein preparation wizard" tool of Schrödinger suite (Schrödinger Release 2021-4: LigPrep, Schrödinger, LLC, New York, NY, USA, 2021) [90]. For this purpose, the bond orders for untemplated residues and known HET groups were assigned and hydrogens were added. Bonds to metals were broken, zero-order bonds between metals and nearby atoms were added, and formal charges to metals and neighboring atoms were corrected. Disulfide bonds were created. Water molecules beyond $5 \AA$ from HET groups were deleted. For ligands, cofactors and metals het states were generated at $\mathrm{pH} 7.0 \pm 2.0$ using LigPrep (Schrödinger Release 2021-4: LigPrep, Schrödinger, LLC, New York, NY, USA, 2021). Finally, H-bonds were optimized by using PROPKA [91] at pH 7.0, water molecules beyond $3 \AA$ from HET groups were removed, and restrained minimization was done using the OPLS4 force field.

\section{Predictions of ADME Properties}

The ADME properties and drug-likeness of selected compounds were determined in terms of distribution, absorption, metabolism, excretion, etc., via the QikProp module of Maestro Schrodinger (Schrödinger Release 2021-4: QikProp, Schrödinger, LLC, New York, NY, USA, 2021).

Receptor Grids Generation and Docking

Glide (Schrödinger Release 2021-4: Glide, Schrödinger, LLC, New York, NY, USA, 2021) was utilized for both grid generation and ligands docking. For docking of fiftythree fungal chaetomugilins and chaetoviridins, three grids were generated using the PDB: $6 \mathrm{M} 2 \mathrm{~N}, 6 \mathrm{~W} 81$, and 7K0f. For the first grid of PDB 6M2N, the binding region was defined by selecting 3WL. For the second grid PDB 6W81, the binding region was defined by selecting X77. For the third grid PDB 7K0f, the binding region was defined by selecting VR4. The non-polar atoms were set for the $\mathrm{VdW}$ radii scaling factor to 1.0 , and the partial charge cut-off was 0.25 . The ligands docking was performed by using the "ligand docking" tool of Schrödinger suite [92]. The selected protocol was standard precision (SP), the ligand sampling method was flexible, and all the other settings were maintained as default.

\section{Simulations of Chaetovirdin D in Complexes with 6M2N, 6W81, and 7K0f}

MD simulations were run using Schrödinger suite (Schrödinger Release 2021-4: Desmond Molecular Dynamics System, D. E. Shaw Research, New York, NY, USA, 2021. Maestro-Desmond Interoperability Tools, Schrödinger, New York, NY, USA, 2021). The systems of 43 in complexes with $6 \mathrm{M} 2 \mathrm{~N}, 6 \mathrm{~W} 81$, and 7K0f were retrieved from docking results and first tuned through the "System Builder" tool. The solvent model TIP3P and then orthorhombic shape box shape were selected. The side distances box was set to $10 \AA$, and the system was neutralized by adding $\mathrm{Na}^{+}$ions. The MD calculations were run for $100 \mathrm{~ns}$ per trajectory, with the number of atoms, pressure, and the temperature kept maintained constant (NPT ensemble). Pressure was set to 1.01325 bar and temperature $300.0 \mathrm{~K}$, and the force field was set as OPLS4.

\section{Biosynthesis of Chaetomugilins and Chaetoviridins}

The biosynthetic studies revealed that these metabolites are generated via a polyketide pathway [93-96]. Their main polyketide chain was produced from malonate and acetate units in a conventional way $[93,95]$. Briefly, a reduced triketide chain (I) was the starting unit to give the aromatic intermediate II, which was processed by the halogenase, followed by hydroxylation-stimulated cyclization catalyzed by monooxygenase to yield cazisochromene (III, chloropyranoquinone) [96]. Then, the addition of an oxidized triketide unit (IV) to III by acyltransferase formed the pyranoquinone V. The latter underwent a Knoevenagel 
reaction, resulting in chaetoviridin A (31). Chaetoviridin A (31) was converted into other chaetomugilins and chaetoviridins through reductions, oxidations, rearrangements, or reactions with amines [93] (Scheme 1).

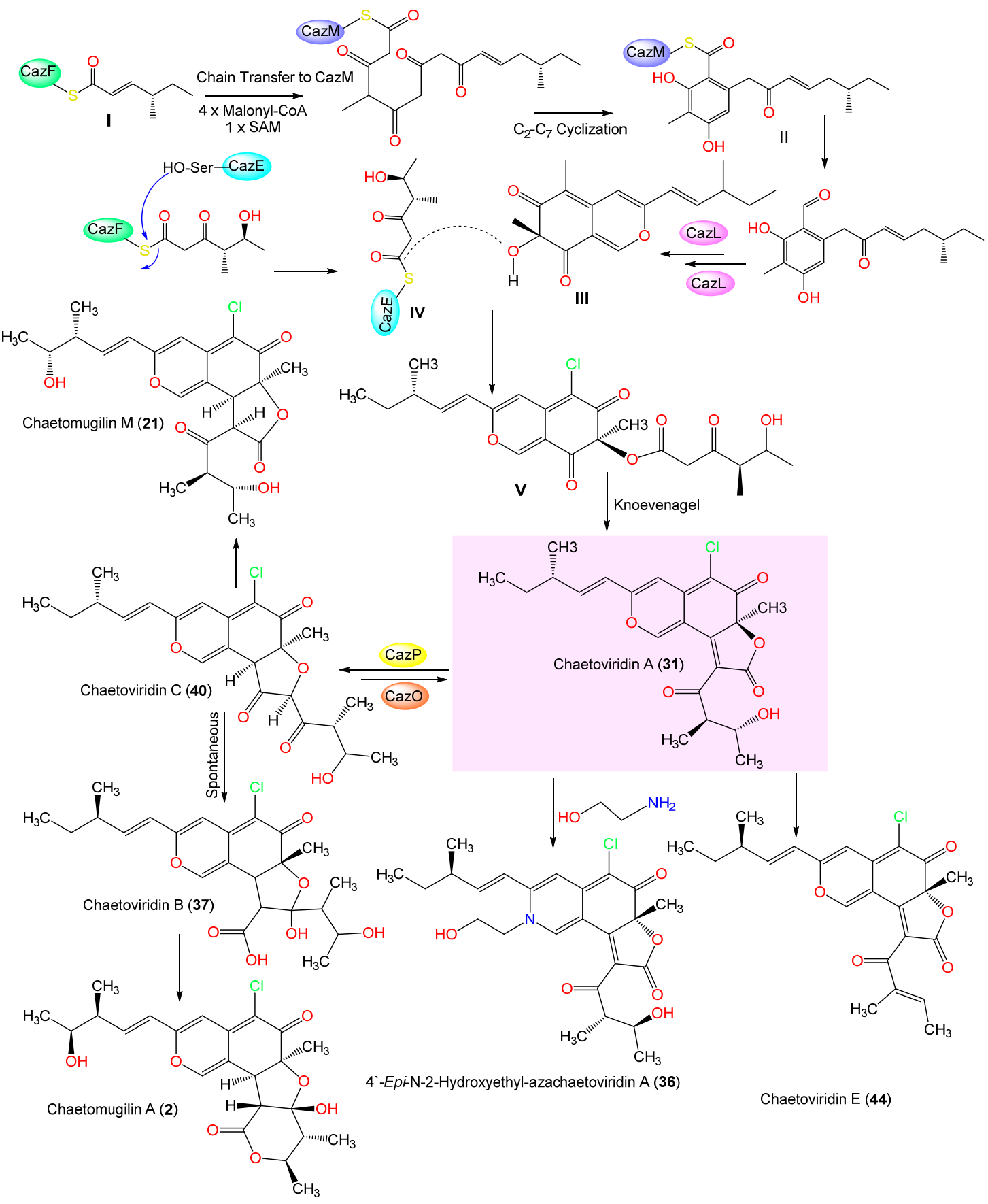

Scheme 1. Proposed biosynthesis of chaetomugilins and chaetoviridins [93-96].

\section{Conclusions}

Fungi are important sources of natural polyketide pharmaceuticals with structural complexities that make them interesting and beneficial biometabolites. Chaetomugilins and chaetoviridins are azaphilone derivatives that are mainly sourced from various Chaetomaceae species. In this work, fifty-six metabolites were isolated from four species, C. globosum, C. cochliodes, C. siamense, C. elatum, and C. subafine, in addition to unidentified Chaetomium species. Most of them were from C. globosum, as shown in Figure 15. The largest quantities 
of these metabolites were found in 2011 (18 compounds), 2009 (16 compounds), and 2017 (14 compounds) (Figure 16).

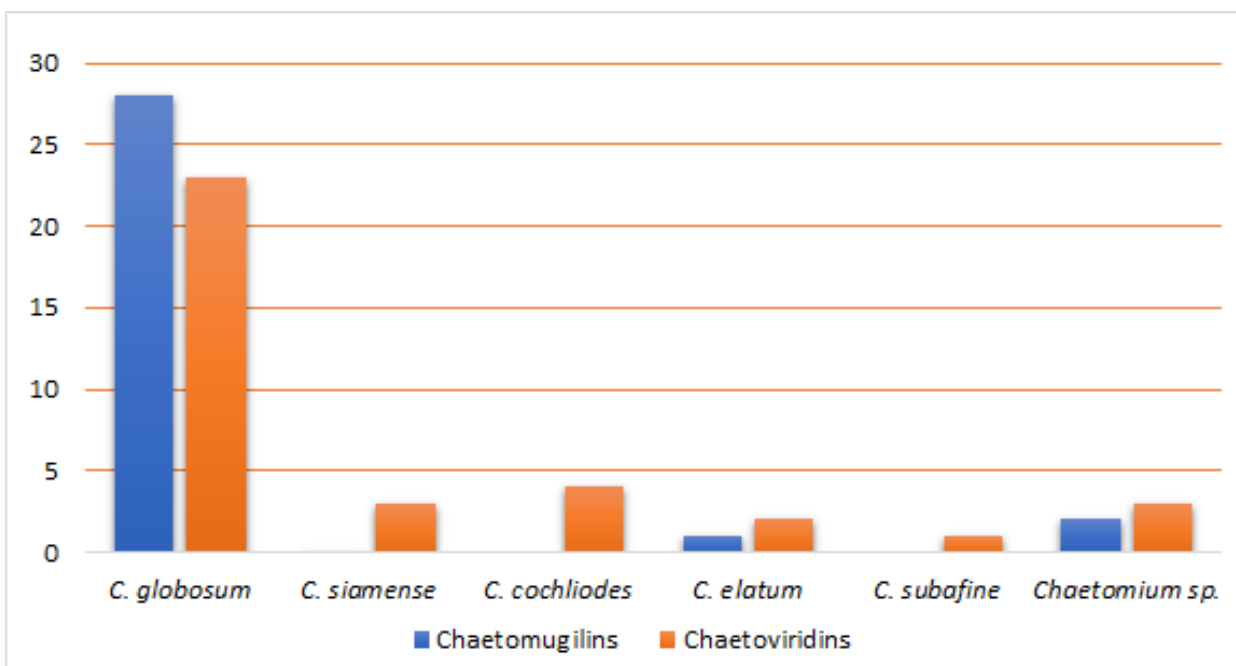

Figure 15. Chaetomugilins and chaetoviridins from various Chaetomium species.

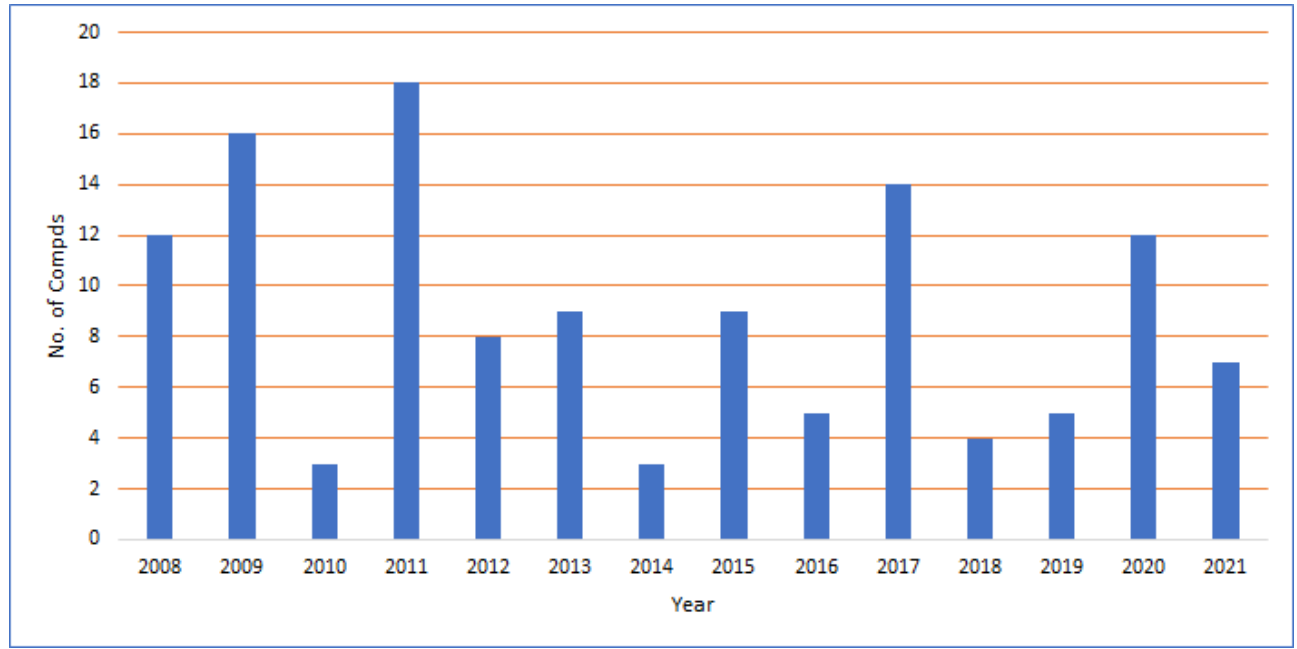

Figure 16. Number of chaetomugilins and chaetoviridins reported per year.

These metabolites have been evaluated for diverse bioactivities, such as cytotoxic, antimicrobial, phytotoxic, antimalarial, anti-mycobacterial, anti-inflammatory, antidiabetic, antioxidant, and antiviral ones; and caspase-3, cholesteryl ester transfer protein, and monoamine oxidase (MAO) inhibitory activities (Figure 17). It was found that some chaetomugilins possessed remarkable cytotoxicity towards certain cancer cell lines, equal to or stronger than the effects of control anticancer drugs, such as chaetomugilins $C$ (7), F (13), I (16), and J (18). 


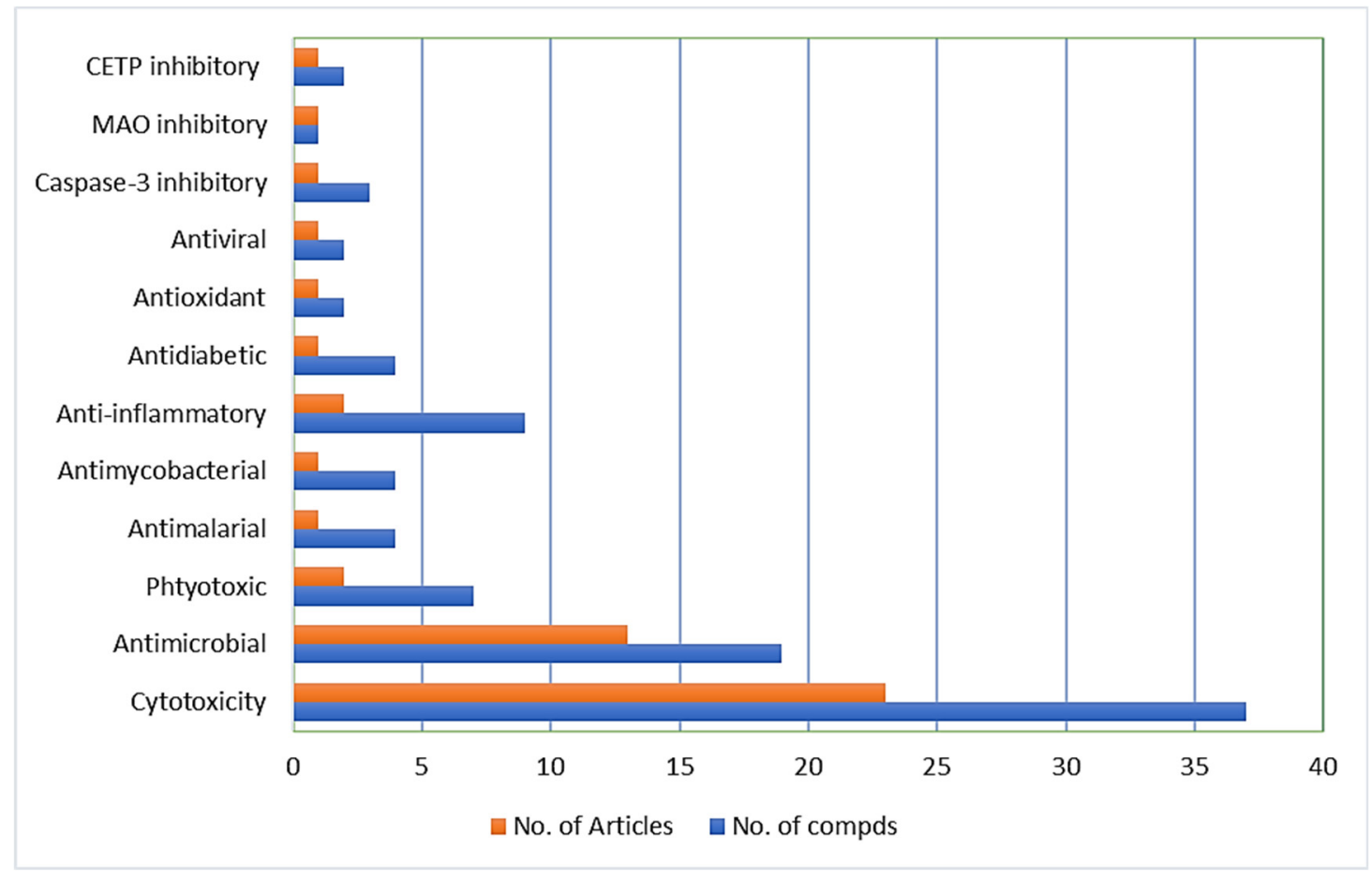

Figure 17. Biological activities of chaetomugilins and chaetoviridins.

Some studies revealed that the use of some additional compounds alongside anticancer drugs increased the sensitivity of the cancer cell lines towards these drugs. These metabolites could be further developed into anticancer agents; however, extensive in vivo studies and explorations of their mechanisms of action are needed. Chaetoviridins, particularly chaetoviridin A (31), have substantial activity towards different plant pathogens; thus, they might be used as biocontrol agents. Additionally, some reports revealed that chaetomugilins (e.g., A (2), D (8), O (23), and S (28)) have serious phytotoxicity, more than the positive controls; therefore, they could be utilized for developing natural eco-friendly herbicides or weedicides that can replace hazardous synthetic compounds. However, extensive studies and field trials should be conducted.

In molecular docking studies, chaetovirdin $\mathrm{D}$ exhibited the highly negative docking scores of $-7.944,-8.141$, and $-6.615 \mathrm{kcal} / \mathrm{mol}$, in complexes with $6 \mathrm{M} 2 \mathrm{~N}, 6 \mathrm{~W} 81$, and $7 \mathrm{~K} 0 \mathrm{f}$ respectively. The reference inhibitor exhibited the following scores: $-5.377,-6.995$, and $-8.159 \mathrm{kcal} / \mathrm{mol}$, in complexes with 6M2N, 6W81, and 7K0f, respectively. By using molecular dynamics simulations, chaetovirdin D's stability in complexes with the viral protease was analyzed, and it was found to be stable over the course of $100 \mathrm{~ns}$ simulation time.

Undoubtedly, chaetomugilins and chaetoviridins are fungi-derived metabolites that have multiple biological activities. They meet all the requirements for becoming drug leads in their respective therapeutic categories. They have varied chemical compositions that might provide the basis for the synthesis and design of novel and effective pharmaceutical agents. The different substituents at various positions in their skeleton play critical roles in the determination of some of their bioactivities. Finally, studies of the possible mechanisms, biosynthetic pathways, structure-activity relationships, and/or derivatization of these metabolites should be the focus of future research.

Author Contributions: Conceptualization, S.R.M.I. and G.A.M.; resources, G.A.M. and S.R.M.I., molecular docking and molecular dynamic, A.M.O.; discussion of the contents, G.A.M., S.R.M.I. and A.M.O., writing - original draft preparation, S.R.M.I., G.A.M. and A.M.O., writing-review and editing, S.R.M.I., G.A.M. and A.M.O. All authors have read and agreed to the published version of the manuscript. 
Funding: The authors extend their appreciation to the Deputyship for Research \& Innovation, Ministry of Education in Saudi Arabia for funding this research work through the project number IFPRP: 200-166-1442 and King Abdulaziz University, DSR, Jeddah, Saudi Arabia.

Institutional Review Board Statement: Not applicable.

Informed Consent Statement: Not applicable.

Data Availability Statement: Not applicable.

Conflicts of Interest: The authors declare no conflict of interest.

\section{References}

1. Mohamed, G.A.; Ibrahim, S.; El-Agamy, D.S.; Elsaed, W.M.; Sirwi, A.; Asfour, H.Z.; Koshak, A.E.; Elhady, S.S. Terretonin as a new protective agent against sepsis-induced acute lung injury: Impact on SIRT1/Nrf2/NF-кBp65/NLRP3 signaling. Biology 2021, 10, 1219. [CrossRef] [PubMed]

2. Mohamed, G.A.; Ibrahim, S.R.M. Untapped potential of marine associated Cladosporium species: An overview on secondary metabolites, biotechnological relevance, and biological activities. Mar. Drugs 2021, 19, 645. [CrossRef] [PubMed]

3. Ibrahim, S.R.M.; Altyar, A.E.; Mohamed, S.G.A.; Mohamed, G.A. Genus Thielavia: Phytochemicals, industrial importance, and biological relevance. Nat. Prod. Res. 2021, 1-16. [CrossRef] [PubMed]

4. Ibrahim, S.R.M.; Mohamed, S.G.A.; Sindi, I.A.; Mohamed, G.A. Biologically active secondary metabolites and biotechnological applications of species of the family Chaetomiaceae (Sordariales): An updated review from 2016 to 2021. Mycol. Prog. 2021, 20, 595-639. [CrossRef]

5. Ibrahim, S.R.M.; Mohamed, S.G.A.; Mohamed, G.A. Humicola genus: Chemical constituents, industrial importance, and biological activities. Curr. Microbiol. 2021. [CrossRef]

6. Ibrahim, S.R.M.; Sirwi, A.; Eid, B.G.; Mohamed, S.G.A.; Mohamed, G.A. Bright Side of Fusarium oxysporum: Secondary Metabolites Bioactivities and Industrial Relevance in Biotechnology and Nanotechnology. J. Fungi 2021, 7, 943. [CrossRef]

7. Ibrahim, S.R.M.; Elkhayat, E.S.; Mohamed, G.A.; Fat'hi, S.M.; Ross, S.A. Fusarithioamide A, a new antimicrobial and cytotoxic benzamide derivative from the endophytic fungus Fusarium chlamydosporium. Biochem. Biophys. Res. Commun. 2016, 479, 211-216. [CrossRef]

8. Ibrahim, S.R.M.; Abdallah, H.M.; Mohamed, G.A.; Ross, S.A. Integracides H.-New tetracyclic triterpenoids from the endophytic fungus Fusarium sp. Fitoterapia 2016, 112, 161-167. [CrossRef]

9. Ibrahim, S.R.M.; Mohamed, G.A.; Ross, S.A. Integracides F and G: New tetracyclic triterpenoids from the endophytic fungus Fusarium sp. Phytochem. Lett. 2016, 15, 125-130. [CrossRef]

10. Ibrahim, S.R.M.; Mohamed, G.A.; Al Haidari, R.A.; El-Kholy, A.A.; Zayed, M.F.; Khayat, M.T. Biologically active fungal depsidones: Chemistry, biosynthesis, structural characterization, and bioactivities. Fitoterapia 2018, 129, 317-365. [CrossRef]

11. Ibrahim, S.R.M.; Mohamed, G.A.; Al Haidari, R.A.; Zayed, M.F.; El-Kholy, A.A.; Elkhayat, E.S.; Ross, S.A. Fusarithioamide B, a new benzamide derivative from the endophytic fungus Fusarium chlamydosporium with potent cytotoxic and antimicrobial activities. Bioorg. Med. Chem. 2018, 26, 786-790. [CrossRef] [PubMed]

12. Ibrahim, S.R.M.; Mohamed, G.A.; Kamal, H.M.K.; Mohamed, S.G.A.; Khedr, A.I.M. Terretonins from Aspergillus Genus: Structures, biosynthesis, bioactivities, and structural elucidation. Mini Rev. Org. Chem. 2021. [CrossRef]

13. Ibrahim, S.R.M.; Sirwi, A.; Eid, B.G.; Mohamed, S.G.A.; Mohamed, G.A. Fungal depsides naturally inspiring molecules: Biosynthesis, structural characterization, and biological activities. Metabolites 2021, 11, 683. [CrossRef]

14. Ibrahim, S.R.M.; Mohamed, G.A.; Khedr, A.M.I. $\gamma$-Butyrolactones from Aspergillus species: Structures, biosynthesis, and biological activities. Nat. Prod. Commun. 2017, 12, 791-800. [CrossRef] [PubMed]

15. Bills, G.F.; Gloer, J.B. Biologically active secondary metabolites from the fungi. Microbiol. Spectr. 2016, 4, 6. [CrossRef] [PubMed]

16. Evidente, A.; Kornienko, A.; Cimmino, A.; Andolfi, A.; Lefranc, F.; Mathieu, V.; Kiss, R. Fungal metabolites with anticancer activity. Nat. Prod. Rep. 2014, 31, 617-627. [CrossRef]

17. Cox, R.J.; Simpson, T.J. Fungal type I polyketide synthases. Methods Enzymol. 2009, 459, 49-78.

18. Simpson, T.J. Fungal polyketide biosynthesis-A personal perspective. Nat. Prod. Rep. 2014, 31, 1247-1252. [CrossRef]

19. Hoffmeister, D.; Keller, N.P. Natural products of filamentous fungi: Enzymes, genes, and their regulation. Nat. Prod. Rep. 2007, 24, 393-416. [CrossRef]

20. Schümann, J.; Hertweck, C. Advances in cloning, functional analysis and heterologous expression of fungal polyketide synthase genes. J. Biotechnol. 2006, 124, 690-703. [CrossRef]

21. Lin, L.; Xu, J. Fungal Pigments and Their Roles Associated with Human Health. J. Fungi 2020, 6, 280. [CrossRef] [PubMed]

22. Sun, C.; Ge, X.; Mudassir, S.; Zhou, L.; Yu, G.; Che, Q.; Zhang, G.; Peng, J.; Gu, Q.; Zhu, T.; et al. New glutamine-containing azaphilone alkaloids from deep-sea-derived fungus Chaetomium globosum HDN151398. Mar. Drugs 2019, 17, 253. [CrossRef] [PubMed]

23. Mapari, S.A.; Meyer, A.S.; Thrane, U. Colorimetric characterization for comparative analysis of fungal pigments and natural food colorants. J. Agric. Food Chem. 2006, 54, 7027-7035. [CrossRef] [PubMed] 
24. Zu, W.Y.; Tang, J.W.; Hu, K.; Zhou, Y.F.; Gou, L.L.; Su, X.Z.; Lei, X.; Sun, H.D.; Puno, P.T. Chaetolactam A, an azaphilone derivative from the endophytic fungus Chaetomium sp. g1. J. Org. Chem. 2021, 86, 475-483. [CrossRef]

25. Wang, W.; Liao, Y.; Chen, R.; Hou, Y.; Ke, W.; Zhang, B.; Gao, M.; Shao, Z.; Chen, J.; Li, F. Chlorinated azaphilone pigments with antimicrobial and cytotoxic activities isolated from the deep sea derived fungus Chaetomium sp. NA-S01-R1. Mar. Drugs 2018, 16, 61. [CrossRef]

26. Makrerougras, M.; Coffinier, R.; Oger, S.; Chevalier, A.; Sabot, C.; Franck, X. Total Synthesis and Structural Revision of Chaetoviridins, A. Org. Lett. 2017, 19, 4146-4149. [CrossRef]

27. Takahashi, M.; Koyama, K.; Natori, S. Four New Azaphilones from Chaetomium globosum var. flavo-viridae. Chem. Pharm. Bull. 1990, 38, 625. [CrossRef]

28. Gao, J.M.; Yang, S.X.; Qin, J.C. Azaphilones: Chemistry and biology. Chem. Rev. 2013, 113, 4755-4811. [CrossRef]

29. Wani, N.A.; Khanday, W.I.; Tirumale, S. Evaluation of anticancer activity of Chaetomium cupreum extracts against human breast adenocarcinoma cell lines. Matrix Sci. Pharma. 2020, 4, 31-40.

30. McMullin, D.R.; Sumarah, M.W.; Miller, J.D. Chaetoglobosins and azaphilones produced by Canadian strains of Chaetomium globosum isolated from the indoor environment. Mycotoxin Res. 2013, 29, 47-54. [CrossRef]

31. Chen, G.D.; Li, Y.J.; Gao, H.; Chen, Y.; Li, X.X.; Li, J.; Guo, L.D.; Cen, Y.Z.; Yao, X.S. New azaphilones and chlorinated phenolic glycosides from Chaetomium elatum with caspase-3 inhibitory activity. Planta Med. 2012, 78, 1683-1689. [CrossRef] [PubMed]

32. Song, C.; Ding, G.; Wu, G.; Yang, J.; Zhang, M.; Wang, H.; Wei, D.; Qin, J.; Guo, L. Identification of a unique azaphilone produced by Chaetomium globosum isolated from Polygonatum sibiricum. Chem. Biodivers. 2020, 17, e1900744. [CrossRef] [PubMed]

33. Chen, C.; Wang, J.; Zhu, H.; Wang, J.; Xue, Y.; Wei, G.; Guo, Y.; Tan, D.; Zhang, J.; Yin, C.; et al. Chaephilones A and B, two new azaphilone derivatives isolated from Chaetomium globosum. Chem. Biodivers. 2016, 13, 422-426. [CrossRef] [PubMed]

34. Hu, X.; Wang, J.; Chai, J.; Yu, X.; Zhang, Y.; Feng, Y.; Qin, J.; Yu, H. Chaetomugilin J enhances apoptosis in human ovarian cancer A2780 cells induced by cisplatin through inhibiting pink1/parkin mediated mitophagy. Onco. Targets Ther. 2020, 13, 9967-9976. [CrossRef]

35. Muroga, Y.; Yamada, T.; Numata, A.; Tanaka, R. 11-and 4-epimers of chaetomugilin a, novel cytostatic metabolit marine fish-derived fungus Chaetomium globosum. Helv. Chim. Acta 2010, 93, 542-549. [CrossRef]

36. Borges, W.S.; Mancilla, G.; Guimarães, D.O.; Durán-Patrón, R.; Collado, I.G.; Pupo, M.T. Azaphilones from the endophyte Chaetomium globosum. J. Nat. Prod. 2011, 74, 1182-1187. [CrossRef]

37. Jinno, M.; Muroga, Y.; Yamada, F.; Tanaka, R. Absolute stereostructures of novel azaphiloness produced by a marine fish-derived fungus. Symp. Chem. Nat. Prod. 2009, 51, 563-568.

38. Yamada, T.; Doi, M.; Shigeta, H.; Muroga, Y.; Hosoe, S.; Numata, A.; Tanaka, R. Absolute stereostructures of cytotoxic metabolites, chaetomugilins A-C, produced by a Chaetomium species separated from a marine fish. Tetrahedron Lett. 2008, 49, 4192-4195. [CrossRef]

39. Yamada, T.; Muroga, Y.; Doi, M.; Shiget, H.; Numata, A.; Tanaka, R. The structures of the cytotoxic metabolites produced by a marine fish-derived fungus. Symp. Chem. Nat. Prod. 2008, 50, 345-350.

40. Yasuhide, M.; Yamada, T.; Numata, A.; Tanaka, R. Chaetomugilins, new selectively cytotoxic metabolites, produced by a marine fish derived Chaetomium species. J. Antibiot. 2008, 61, 615-622. [CrossRef]

41. Yamada, T.; Yasuhide, M.; Shigeta, H.; Numata, A.; Tanaka, R. Absolute stereostructures of chaetomugilins G and H produced by a marine-fish-derived Chaetomium species. J. Antibiot. 2009, 62, 353-357. [CrossRef] [PubMed]

42. Qin, J.C.; Zhang, Y.M.; Gao, J.M.; Bai, M.S.; Yang, S.X.; Laatsch, H.; Zhang, A.L. Bioactive metabolites produced by Chaetomium globosum, an endophytic fungus isolated from Ginkgo biloba. Bioorg. Med. Chem. Lett. 2009, 19, 1572-1574. [CrossRef] [PubMed]

43. Yamada, T.; Jinno, M.; Kikuchi, T.; Kajimoto, T.; Numata, A.; Tanaka, R. Three new azaphilones produced by a marine fish derived Chaetomium globosum. J. Antibiot. 2012, 65, 413-417. [CrossRef] [PubMed]

44. Zhang, C.-Y.; Ji, X.; Gui, X.; Huang, B.-K. Chemical constituents from an endophytic fungus Chaetomium globosum Z1. Nat. Prod. Commun. 2013, 8, 1217-1218. [CrossRef] [PubMed]

45. Wang, D.; Zhang, Y.; Li, X.; Pan, H.; Chang, M.; Zheng, T.; Sun, J.; Qiu, D.; Zhang, M.; Wei, D.; et al. Potential allelopathic azaphilones produced by the endophytic Chaetomium globosum TY1 inhabited in Ginkgo biloba using the one strain-many compounds method. Nat. Prod. Res. 2017, 31, 724-728. [CrossRef]

46. Wang, M.H.; Jiang, T.; Ding, G.; Niu, S.B.; Wang, X.W.; Yu, M.; Gu, Y.C.; Zhang, Q.B.; Chen, J.H.; Jia, H.M.; et al. Molecular epigenetic approach activates silent gene cluster producing dimeric bis-spiro-azaphilones in Chaetomium globosum CBS148.51. J. Antibiot. 2017, 70, 801-804. [CrossRef] [PubMed]

47. Qi, J.; Wang, D.; Yin, X.; Zhang, Q.; Gao, J.-M. New metabolite with inhibitory activity against $\alpha$-glucosidase and $\alpha$-amylase from endophytic Chaetomium globosum. Nat. Prod. Commun. 2020, 15, 1-9. [CrossRef]

48. Yamada, T.; Muroga, Y.; Tanaka, R. New azaphilones, seco-chaetomugilins A and D, produced by a marine-fish-derived Chaetomium globosum. Mar. Drugs 2009, 7, 249-257. [CrossRef]

49. Selim, K.A.M.A. Biological Evaluation and Chemical Identification of Secondary Metabolites of Endophytic Fungi from Egyptian Flora. Master's Thesis, Faculty of Science, Cairo University, Cairo, Egypt, 2008.

50. McMullin, D.R.; Sumarah, M.W.; Blackwell, B.A.; Miller, J.D. New azaphilones from Chaetomium globosum isolated from the built environment. Tetrahedron Lett. 2013, 54, 568-572. [CrossRef] 
51. Piyasena, K.G.N.P.; Wickramarachchi, W.A.R.T.; Kumar, N.S.; Jayasinghe, L.; Fujimoto, Y. Two phytotoxic azaphilone derivatives from Chaetomium globosum, a fungal endophyte isolated from Amaranthus viridis leaves. Mycology 2015, 6, 158-160. [CrossRef]

52. Rand, T.G.; Chang, C.T.; McMullin, D.R.; Miller, J.D. Inflammation-associated gene expression in RAW 264.7 macrophages induced by toxins from fungi common on damp building materials. Toxicol. In Vitro 2017, 43, 16-20. [CrossRef] [PubMed]

53. Salo, J.M.; Kedves, O.; Mikkola, R.; Kredics, L.; Andersson, M.A.; Kurnitski, J.; Salonen, H. Detection of Chaetomium globosum, $\mathrm{Ch}$. Cochliodes and Ch. Rectangulare during the diversity tracking of mycotoxin-producing Chaetomium-like isolates obtained in buildings in Finland. Toxins 2020, 12, 443. [CrossRef] [PubMed]

54. Ryu, Y. Cytochalasan and Azaphilone Derivatives from a Marine-Derived Fungus Chaetomium globosum. Master's Thesis, Seoul National University, Seoul, Korea, 2017.

55. McMullin, D.R. Structural Characterization of Secondary Metabolites Produced by Fungi Obtained from Damp Canadian Buildings. Ph.D. Thesis, Carleton University, Ottawa, ON, Canada, 2014.

56. Youn, U.J.; Sripisut, T.; Park, E.J.; Kondratyuk, T.P.; Fatima, N.; Simmons, C.J.; Wall, M.M.; Sun, D.; Pezzuto, J.M.; Chang, L.C. Determination of the absolute configuration of chaetoviridins and other bioactive azaphilones from the endophytic fungus Chaetomium globosum. Bioorg. Med. Chem. Lett. 2015, 25, 4719-4723. [CrossRef]

57. Kumar, R.; Kundu, A.; Dutta, A.; Saha, S.; Das, A.; Bhowmik, A. Chemo-profiling of bioactive metabolites from Chaetomium globosum for biocontrol of Sclerotinia rot and plant growth promotion. Fungal Biol. 2021, 125, 167-176. [CrossRef] [PubMed]

58. Muroga, Y.; Yamada, T.; Numata, A.; Tanaka, R. Chaetomugilins I-O, new potent cytotoxic metabolites from a marine-fish-derived Chaetomium species. Stereochemistry and biological activities. Tetrahedron 2009, 65, 7580-7586. [CrossRef]

59. Yamada, T.; Muroga, Y.; Jinno, M.; Kajimoto, T.; Usami, Y.; Numata, A.; Tanaka, R. New class azaphilone produced by a marine fish derived Chaetomium globosum. The stereochemistry and biological activities. Bioorg. Med. Chem. 2011, 19, 4106-4113. [CrossRef]

60. Phonkerd, N.; Kanokmedhakul, S.; Kanokmedhakul, K.; Soytong, K.; Prabpai, S.; Kongsearee, P. Bio-spiro-azaphilones and azaphilones from the fungi Chaetomium cochliodes VTh01 and C. cochliodes Cth05. Tetrahedron 2008, 64, 9636-9645. [CrossRef]

61. Park, J.H.; Choi, G.J.; Jang, K.S.; Lim, H.K.; Kim, H.T.; Cho, K.Y.; Kim, J.C. Antifungal activity against plant pathogenic fungi of chaetoviridins isolated from Chaetomium globosum. FEMS Microbiol. Lett. 2005, 252, 309-313. [CrossRef]

62. Pornsuriya, C.; Soytong, K.; Poeaim, S.; Kanokmedhakul, S.; Khumkomkhet, P.; Lin, F.-C.; Wang, H.K.; Hyde, K.D. Chaetomium siamense sp. nov., a soil isolate from Thailand, produces a new chaetoviridin, G. Mycotaxon 2011, 115, 19-27. [CrossRef]

63. Awad, N.E.; Kassem, H.A.; Hamed, M.A.; El-Naggar, M.A.; El-Feky, A.M. Bioassays guided isolation of compounds from Chaetomium globosum. J. Mycol. Med. 2014, 24, e35-e42. [CrossRef]

64. Xu, G.-B.; Liu, J.; Xiao, X.; Zhou, M.; Yan, T.; Li, G.-Y. Isolation and identification of secondary metabolites from Chaetomium globosum CIB-160 and their immunological activity. Nat. Prod. Res. Dev. 2016, 28, 1562-1567.

65. Koyama, K.; Takahashi, K.; Natori, S.; Udagawa, S. Production of mycotoxins by Chaetomium species. Proc. Jpn. Assoc. Mycotoxicol. 1991, 33, 40-43. [CrossRef]

66. Fierro-Cruz, J.E.; Jiménez, P.; Coy-Barrera, E. Fungal endophytes isolated from Protium heptaphyllum and Trattinnickia rhoifolia as antagonists of Fusarium oxysporum. Rev. Argent. Microbiol. 2017, 49, 255-263. [CrossRef] [PubMed]

67. Yue, H.M.; Wang, M.; Gong, W.F.; Zhang, L.Q. The screening and identification of the biological control fungi Chaetomium spp. against wheat common root rot. FEMS Microbiol. Lett. 2018, 365, 242. [CrossRef] [PubMed]

68. Zhang, X.Y.; Tan, X.M.; Yu, M.; Yang, J.; Sun, B.D.; Qin, J.C.; Guo, L.P.; Ding, G. Bioactive metabolites from the desert plantassociated endophytic fungus Chaetomium globosum (Chaetomiaceae). Phytochemistry 2021, 185, 112701. [CrossRef] [PubMed]

69. Zhang, Y.; Zhu, H.; Ye, Y.; Tang, C. Antifungal activity of chaetoviridin A from Chaetomium globosum CEF-082 metabolites against Verticillium dahliae in cotton. Mol. Plant Microbe Interact. 2021, 34, 758-769. [CrossRef]

70. Qi, J.; Jiang, L.; Zhao, P.; Chen, H.; Jia, X.; Zhao, L.; Dai, H.; Hu, J.; Liu, C.; Shim, S.H.; et al. Chaetoglobosins and azaphilones from Chaetomium globosum associated with Apostichopus japonicus. Appl. Microbiol. Biotechnol. 2020, 104, 1545-1553. [CrossRef]

71. Wang, W.; Yang, J.; Liao, Y.Y.; Cheng, G.; Chen, J.; Cheng, X.D.; Qin, J.J.; Shao, Z. Cytotoxic nitrogenated azaphilones from the deep-sea-derived fungus Chaetomium globosum MP4-S01-7. J. Nat. Prod. 2020, 83, 1157-1166. [CrossRef]

72. Li, X.; Tian, Y.; Yang, S.X.; Zhang, Y.M.; Qin, J.C. Cytotoxic azaphilone alkaloids from Chaetomium globosum TY1. Bioorg. Med. Chem. Lett. 2013, 23, 2945-2947. [CrossRef]

73. Yan, W.; Cao, L.L.; Zhang, Y.Y.; Zhao, R.; Zhao, S.S.; Khan, B.; Ye, Y.H. New metabolites from endophytic fungus Chaetomium globosum CDW7. Molecules 2018, 23, 2873. [CrossRef]

74. Kingsland, S.R.; Barrow, R.A. Identification of chaetoviridin E from a cultured microfungus, Chaetomium sp. and structural reassignment of chaetoviridins B and D. Aust. J. Chem. 2009, 62, 269-274. [CrossRef]

75. Steyn, P.S.; Vleggaar, R. The Structure of Dihydrodeoxy-8-epi-austdiol and the Absolute Configuration of the Azaphilones. J. Chem. Soc. Perkin Trans. 1976, 7, 204-206. [CrossRef]

76. Yasukawa, K.; Takahashi, M.; Natori, S.; Kawai, K.; Yamazaki, M.; Takeuchi, M.; Takido, M. Azaphilones inhibit tumor promotion by 12-O-tetradecanoylphorbol-13-acetate in two-stage carcinogenesis in mice. Oncology 1994, 51, 108-112. [CrossRef] [PubMed]

77. Mehmet, H. Caspases find a new place to hide. Nature 2000, 403, 29-30. [CrossRef]

78. Howley, B.; Fearnhead, H.O. Caspases as therapeutic targets. J. Cell. Mol. Med. 2008, 12, 1502-1516. [CrossRef]

79. Taheri, H.; Filion, K.B.; Windle, S.B.; Reynier, P.; Eisenberg, M.J. Cholesteryl ester transfer protein inhibitors and cardiovascular outcomes: A systematic review and meta-analysis of randomized controlled trials. Cardiology 2020, 145, 236-250. [CrossRef] 
80. Tomoda, H.; Matsushima, C.; Tabata, N.; Namatame, I.; Tanaka, H.; Bamberger, M.J.; Arai, H.; Fukazawa, M.; Inoue, K.; Omura, S. Structure-specific inhibition of cholesteryl ester transfer protein by azaphilones. J. Antibiot. 1999, 52, 160-170. [CrossRef]

81. Acter, T.; Uddin, N.; Das, J.; Akhter, A.; Choudhury, T.R.; Kim, S. Evolution of severe acute respiratory syndrome coronavirus 2 (SARS-CoV-2) as coronavirus disease 2019 (COVID-19) pandemic: A global health emergency. Sci. Total Environ. 2020, 730, 138996. [CrossRef]

82. Abdallah, H.M.; El-Halawany, A.M.; Sirwi, A.; El-Araby, A.M.; Mohamed, G.A.; Ibrahim, S.R.M.; Koshak, A.E.; Asfour, H.Z.; Awan, Z.A.; Elfaky, M.A. Repurposing of some natural product isolates as SARS-COV-2 main protease inhibitors via in vitro cell-free and cell-based antiviral assessments and molecular modeling approaches. Pharmaceuticals 2021, 14, 213. [CrossRef]

83. Vellingiri, B.K.; Jayaramayya, M.; Iyer, A.; Narayanasamy, V.; Govindasamy, B.; Giridharan, S.; Ganesan, A.; Venugopal, D.; Venkatesan, H.; Ganesan, K.; et al. Subramaniam, COVID-19: A promising cure for the global panic. Sci. Total Environ. 2020, 725, 138277. [CrossRef]

84. Zarei, A.; Fardood, S.T.; Moradnia, F.; Ramazani, A. A review on coronavirus family persistency and considerations of novel type, COVID-19 features. Eurasian Chem. Commun. 2020, 2, 798-811. [CrossRef]

85. Anand, K.; Ziebuhr, J.; Wadhwani, P.; Mesters, J.R.; Hilgenfeld, R. Coronavirus main proteinase (3CLpro) structure: Basis for design of anti-SARS drugs. Science 2003, 300, 1763-1767. [CrossRef] [PubMed]

86. Vandyck, K.; Abdelnabi, R.; Gupta, K.; Jochmans, D.; Jekle, A.; Deval, J.; Misner, D.; Bardiot, D.; Foo, C.S.; Liu, C.; et al. ALG-097111, a potent and selective SARS-CoV-2 3-chymotrypsin-like cysteine protease inhibitor exhibits in vivo efficacy in a Syrian Hamster model. Biochem. Biophys. Res. Commun. 2021, 555, 134-139. [CrossRef] [PubMed]

87. Yang, H.; Xie, W.; Xue, X.; Yang, K.; Ma, J.; Liang, W. Design of wide-spectrum inhibitors targeting coronavirus main proteases. PLoS Biol. 2005, 3, e324.

88. Fiorucci, D.; Milletti, E.; Orofino, F.; Brizzi, A.; Mugnaini, C.; Corelli, F. Computational drug repurposing for the identification of SARS-CoV-2 main protease inhibitors. J. Biomol. Struct. Dyn. 2021, 39, 6242-6248. [CrossRef]

89. Linnakoski, R.; Reshamwala, D.; Veteli, P.; Cortina-Escribano, M.; Vanhanen, H.; Marjomäki, V. Antiviral agents from fungi: Diversity, mechanisms and potential applications. Front. Microbiol. 2018, 9, 2325. [CrossRef]

90. Sastry, M.G.; Adzhigirey, M.; Day, T.; Annabhimoju, R.; Sherman, W. Protein and ligand preparation: Parameters, protocols, and influence on virtual screening enrichments. J. Comput. Aided Mol. Des. 2013, 27, 221-234. [CrossRef]

91. Olsson, M.H.M.; Søndergaard, C.R.; Rostkowski, M.; Jensen, J.H. PROPKA3: Consistent Treatment of Internal and Surface Residues in Empirical pK a Predictions. J. Chem. Theory Comput. 2011, 7, 525-537. [CrossRef]

92. Friesner, R.A.; Banks, J.L.; Murphy, R.B.; Halgren, T.A.; Klicic, J.J.; Mainz, D.T.; Repasky, M.P.; Knoll, E.H.; Shelley, M.; Perry, J.K.; et al. Glide: A New Approach for Rapid, Accurate Docking and Scoring. 1. Method and Assessment of Docking Accuracy. J. Med. Chem. 2004, 47, 1739-1749. [CrossRef]

93. Pavesi, C.; Flon, V.; Mann, S.; Leleu, S.; Prado, S.; Franck, X. Biosynthesis of azaphilones: A review. Nat. Prod. Rep. 2021, 38, 1058-1071. [CrossRef]

94. Winter, J.M.; Sato, M.; Sugimoto, S.; Chiou, G.; Garg, N.K.; Tang, Y.; Watanabe, K. Identification and characterization of the chaetoviridin and chaetomugilin gene cluster in Chaetomium globosum reveals dual functions of an iterative highly reducing polyketide synthase. J. Am. Chem. Soc. 2012, 134, 17900-17903. [CrossRef] [PubMed]

95. Winter, J.M.; Cascio, D.; Dietrich, D.; Sato, M.; Watanabe, K.; Sawaya, M.R.; Vederas, J.C.; Tang, Y. Biochemical and structural basis for controlling chemical modularity in fungal polyketide biosynthesis. J. Am. Chem. Soc. 2015, 137, 9885-9893. [CrossRef] [PubMed]

96. Sato, M.; Winter, J.M.; Kishimoto, S.; Noguchi, H.; Tang, Y.; Watanabe, K. Combinatorial generation of chemical diversity by redox enzymes in chaetoviridin biosynthesis. Org. Lett. 2016, 18, 1446-1449. [CrossRef] [PubMed] 